\title{
DIFFRACTIVE GEOMETRIC OPTICS FOR BLOCH WAVE PACKETS
}

\author{
GRÉGOIRE ALLAIRE ${ }^{1}$, MARIAPIA PALOMBARO $^{2}$, AND JEFFREY RAUCH ${ }^{3}$
}

\begin{abstract}
We study, for times of order $1 / \varepsilon$, solutions of wave equations which are $\mathcal{O}\left(\varepsilon^{2}\right)$ modulations of an $\varepsilon$ periodic wave equation. The solutions are of slowly varying amplitude type built on Bloch plane waves with wavelength of order $\varepsilon$. We construct accurate approximate solutions of three scale WKB type. The leading profile is both transported at the group velocity and dispersed by a Schrödinger equation given by the quadratic approximation of the Bloch dispersion relation at the plane wave. A ray average hypothesis of small divisor type guarantees stability. We introduce techniques related to those developed in nonlinear geometric optics which lead to new results even on times scales $t=\mathcal{O}(1)$. A pair of asymptotic solutions yield accurate approximate solutions of oscillatory initial value problems. The leading term yields $H^{1}$ asymptotics when the envelopes are only $H^{1}$.
\end{abstract}

Key words: Geometric optics, diffractive geometric optics, Bloch waves, diffraction, homogenization.

2000 Mathematics Subject Classification: 35B40, 35B27, 35L30, 35B34, $35 \mathrm{~J} 10$.

${ }^{1}$ Centre de Mathématiques Appliquées, École Polytechnique, 91128 Palaiseau, France.

Email: gregoire.allaire@polytechnique.fr

${ }^{2}$ Centro di Ricerca Matematica Ennio De Giorgi, Scuola Normale Superiore, Piazza dei

Cavalieri 3, I-56100 Pisa, Italy. Email: mariapia.palombaro@sns.it

${ }^{3}$ Department of Mathematics, University of Michigan, Ann Arbor 48109 MI, USA.

Email: rauch@umich.edu

\section{INTRODUCTION}

This paper studies the propagation of waves through a slightly perturbed periodic medium. The period $\varepsilon$ is assumed to be small compared to the size of the wave packet that we take as $\mathcal{O}(1), \varepsilon<<1$. The equations are hyperbolic and the Cauchy problem is solvable for arbitrary initial data. The wavelength of solutions is determined by the initial data. We study the delicate case where the wavelength $\ell$ and period are small and of comparable size. As discussed below, this scaling is particularly important in technology.

The resonant case $\ell \sim \varepsilon$ contrasts with the case of waves with wavelength large compared to the period of the medium, $\ell>>\varepsilon$. For such long waves, the medium can, with small error, be replaced by a medium which does not vary on the small scale (see e.g. 
[8], [10]). The homogenized limit is a wave equation with effective coefficients that are computed as in the static case. The pertinent dispersion relation and group velocities are those of the homogenized equations. For a second order scalar equation, the dispersion relation is quadratic in frequency and wave number as is the dispersion relation of the original problem.

If $\ell<<\varepsilon$, then from the point of view of the wave, the medium is slowly varying and the approximations of standard geometric optics are appropriate. The group velocities are those defined by the characteristic variety of the equation with nearly periodic coefficients. For second order scalar equations the dispersion relation is quadratic with coefficients which vary on the short scale $\varepsilon$.

In this paper we discuss the resonant case when $\ell \sim \varepsilon$. A principal interest of this scaling is that the dispersion relation (1.6) is given in terms of Bloch eigenvalues, denoted below by $\lambda_{n}$. It can be very different from the relations in the preceding regimes. For example, for scalar second order equations the group velocity has strictly positive norm, while in the resonant case there can be zero speeds. Periodic structures are the focus of intensive work on designer photonic materials. Since for $\ell \sim \varepsilon$ the dispersion relation can have form entirely different from the original equations, this leaves open the door for materials with radically different properties than the periodic constituents. Among goals achieved by such efforts is to slow light ([21], [5], [36], [20], [3]), and to achieve preassigned band gap structures. The use of the latter materials in optimized fibers is now common practice (see [30]). It is dreamed that the slow light technologies are a first step toward an all optical computer.

In the case when $\ell \sim \varepsilon$ and for times $t \sim 1$, there is a geometric optics approximation with propagation speeds given by group velocities defined from the Bloch spectral theory (see [11], [8], $§ 2$ and $\S 3$ below, and [18], [19] for a Wigner measure approach). Our main results concern the propagation of such Bloch wave packets on the long time scales $t \sim \varepsilon^{-1}$ associated with diffractive geometric optics. For these long times, the supports of solutions extend beyond the tube of rays with feet in the support of the initial data. The behavior is described by Schrödinger equations whose dispersion is computed from Bloch spectral data and whose lower order (potential) term is obtained by an averaging of the small perturbations of the periodic medium. We give an infinitely accurate analysis at the scale of geometric optics, and a mathematically solid foundation at the scale of diffractive geometric optics.

We consider the following wave equation describing an $\mathcal{O}\left(\varepsilon^{2}\right)$ perturbation of a $\varepsilon$ periodic medium

$$
P^{\varepsilon}\left(t, x, \partial_{t, x}\right) u^{\varepsilon}:=\rho^{\varepsilon} \frac{\partial^{2} u^{\varepsilon}}{\partial t^{2}}-\operatorname{div}\left(A^{\varepsilon} \operatorname{grad} u^{\varepsilon}\right)=0 \quad \text { in } \quad\left[0, \infty\left[\times \mathbb{R}_{x}^{N}\right.\right.
$$


The coefficients $A^{\varepsilon}$ and $\rho^{\varepsilon}$ are of the form

$$
A^{\varepsilon}(x)=A_{0}\left(\frac{x}{\varepsilon}\right)+\varepsilon^{2} A_{1}\left(t, x, \frac{x}{\varepsilon}\right), \quad \rho^{\varepsilon}(x)=\rho_{0}\left(\frac{x}{\varepsilon}\right)+\varepsilon^{2} \rho_{1}\left(t, x, \frac{x}{\varepsilon}\right) .
$$

The unperturbed coefficients $A_{0}(x / \varepsilon)$ and $\rho_{0}(x / \varepsilon)$ are periodic with period $\varepsilon$. We suppose that $\rho_{0}(y)$ and $\rho_{1}(t, x, y)$ are smooth real valued functions on $\mathbb{T}_{y}^{N}:=\mathbb{R}^{N} / \mathbb{Z}^{N}$ (the flat unit torus) and $\mathbb{R}^{1+N} \times \mathbb{T}_{y}^{N}$ respectively. The functions $A_{0}(y)$ and $A_{1}(t, x, y)$ are smooth symmetric matrix valued functions on $\mathbb{T}_{y}^{N}$ and $\mathbb{R}^{1+N} \times \mathbb{T}_{y}^{N}$ respectively. For each $\alpha, j$, we assume

$$
\left\{\partial_{t, x, y}^{\alpha} \rho_{j}, \partial_{t, x, y}^{\alpha} A_{j}\right\} \in L^{\infty}\left(\mathbb{R}_{t, x}^{1+N} \times \mathbb{T}_{y}^{N}\right)
$$

There is a constant $\delta>0$ so that for all $y$,

$$
\rho_{0}(y) \geq \delta>0, \quad A_{0}(y) \geq \delta I>0 .
$$

The scaling in (1.2) is such that the $\mathcal{O}\left(\varepsilon^{2}\right)$ perturbations affect the leading term of the approximate solutions for $t=\mathcal{O}(1 / \varepsilon)$. Smaller perturbations, that is with a higher power of $\varepsilon$, would not affect the leading order approximation for times of order $1 / \varepsilon$.

Remark 1.1. The time derivative in (1.1) is not taken in the divergence form $\partial_{t}\left(\rho \partial_{t}\right)$. The two forms are equivalent for time independent coefficients. When there are modulations in time the proofs of accuracy are a little easier in the divergence form case. Our earlier article [4] gives the formulas for the divergence form case. The $\partial_{t}^{2}(\rho u)$ form is of difficulty equal to the present case requiring no ideas not already present. We chose to do one of the hard cases so as to make such a statement.

In the purely periodic case, with $\rho_{1}$ and $A_{1}$ identically equal to zero, solutions are linear combinations of Bloch plane wave solutions (see $\S 2.1$ ),

$$
e^{2 \pi i(\omega(\theta) t+\theta . x) / \varepsilon} \psi_{n}(x / \varepsilon, \theta), \quad \theta \in\left[0,1\left[^{N},\right.\right.
$$

with $\omega$ satisfying the dispersion relation,

$$
4 \pi^{2} \omega^{2}(\theta)=\lambda_{n}(\theta) .
$$

The Bloch eigenfunction, $\psi_{n}(y, \theta)$, is 1-periodic in $y$ and satisfies the eigenvalue equation,

$$
-\left(\operatorname{div}_{y}+2 i \pi \theta\right)\left(A_{0}(y)\left(\operatorname{grad}_{y}+2 i \pi \theta\right) \psi_{n}\right)=\lambda_{n}(\theta) \rho_{0}(y) \psi_{n} \quad \text { in } \quad \mathbb{T}_{y}^{N},
$$

corresponding to the $n$-th eigenvalue or energy level $\lambda_{n}(\theta)$. Equation (1.7) together with (1.6) is equivalent to the wave equation (1.1) for the plane wave solution (1.5). The Hilbert space $L^{2}\left(\mathbb{T}_{y}^{N}\right)$ is normed by

$$
\|\psi\|_{L^{2}\left(\mathbb{T}^{N}\right)}^{2}:=\int_{\mathbb{T}^{N}}|\psi(y)|^{2} d y .
$$


The operator on the left in (1.7) is hermitian symmetric in the associated scalar product. The eigenfunctions corresponding to distinct eigenvalues are orthogonal with respect to the scalar product of the equivalent norm,

$$
\left(\int \rho_{0}(y)|\psi(y)|^{2} d y\right)^{1 / 2}
$$

Definition 1.2. Fix $\theta_{0}$ and a simple eigenvalue $\lambda_{n}\left(\theta_{0}\right) \neq 0$ and $\omega$ one of the roots of (1.6). Denote by $\mathbb{K} \subset L^{2}\left(\mathbb{T}_{y}^{N}\right)$ the one dimensional eigenspace, and by $\psi_{n}\left(y, \theta_{0}\right)$ an eigenfunction normalized with respect to the $L^{2}\left(\mathbb{T}_{y}^{N}, \rho_{0}(y) d y\right)$ scalar product,

$$
\int \rho_{0}(y)\left|\psi_{n}\left(y, \theta_{0}\right)\right|^{2} d y=1
$$

Denote by $\Pi$ the $L^{2}\left(\mathbb{T}_{y}^{N}, d y\right)$ orthogonal projection onto $\mathbb{K}$.

In a neighborhood of $\theta_{0}, \lambda_{n}(\theta)$ is thus a well defined simple eigenvalue and $\lambda_{n}, \omega$ are analytic functions of $\theta$. The group velocity is defined as,

$$
\mathcal{V}:=-\nabla_{\theta} \omega\left(\theta_{0}\right) \text {. }
$$

We construct approximate solutions of (1.1) which have a linear phase,

$$
S(t, x):=\omega\left(\theta_{0}\right) t+\theta_{0} \cdot x .
$$

Our main results show that the $\mathcal{O}\left(\varepsilon^{2}\right)$ perturbations affect the leading asymptotics for times $t$ of order $1 / \varepsilon$ while perturbations $\mathcal{O}(\varepsilon)$ affect the leading behavior at times $t$ of order 1 . The rule of thumb is that the time of influence of the perturbations is of order $\varepsilon$ divided by the amplitude of the perturbations.

We first describe the geometric optics approximation for times $t=\mathcal{O}(1)$. In such a case, it is possible to consider larger perturbations of order $\mathcal{O}(\varepsilon)$, namely to replace (1.2) by

$$
A^{\varepsilon}(x)=A_{0}\left(\frac{x}{\varepsilon}\right)+\varepsilon A_{1}\left(t, x, \frac{x}{\varepsilon}\right), \quad \rho^{\varepsilon}(x)=\rho_{0}\left(\frac{x}{\varepsilon}\right)+\varepsilon \rho_{1}\left(t, x, \frac{x}{\varepsilon}\right) .
$$

In Section 3 we construct infinitely accurate approximate solutions $v^{\varepsilon}$ for problem (1.1) which are of Bloch wave type with slowly varying amplitude, (1.11) $v^{\varepsilon}(t, x):=e^{2 \pi i S / \varepsilon} W(\varepsilon, t, x, x / \varepsilon), \quad W(\varepsilon, t, x, y) \sim w_{0}(t, x, y)+\varepsilon w_{1}(t, x, y)+\ldots$, where $S$ is the linear phase (1.10). The $\sim$ is in the sense of Taylor expansion in $\varepsilon$,

$$
w_{j}=\frac{1}{j !} \frac{\partial^{j} W(0, t, x, y)}{\partial \varepsilon^{j}} .
$$

It is an asymptotic expansion as $\varepsilon \rightarrow 0$, not a convergent infinite series. For any $m$,

$$
W(\varepsilon, t, x, y)-\sum_{j=0}^{m} \varepsilon^{j} w_{j}(t, x, y)=\mathcal{O}\left(\varepsilon^{m+1}\right), \quad \text { as } \quad \varepsilon \rightarrow 0 .
$$


The expansion (1.11) is inserted in $P^{\varepsilon} v^{\varepsilon}$ and terms grouped by powers of $\varepsilon$. To make the largest term vanish, the leading profile $w_{0}(t, x, \cdot)$ must be a $\mathbb{K}$ valued function of $(t, x)$. Equivalently, the leading order term in the approximate solution (1.11) is of the form

$$
e^{2 \pi i S / \varepsilon} a(t, x) \psi_{n}\left(x / \varepsilon, \theta_{0}\right), \quad a \in C^{\infty} .
$$

It is a Bloch plane wave with slowly varying amplitude $a(t, x)$. Equation (1.7) is written as $\mathbb{L} w_{0}=0$. The equation at each order in $\varepsilon$ in the expansion of $P^{\varepsilon} v^{\varepsilon}$ is projected in turn onto the kernel and the range of the operator $\mathbb{L}$ which is neither injective nor surjective. This yields equations which determine the profiles $w_{j}$. For example, the profile $w_{0}=a \psi_{n}$ is determined from its initial data by the transport equation (see $\S 3$ or [8], [18]),

$$
\left(\partial_{t}+\mathcal{V} . \partial_{x}\right) w_{0}=0, \quad \text { equivalently } \quad\left(\partial_{t}+\mathcal{V} . \partial_{x}\right) a=0
$$

Therefore the function $w_{0}$ is constant on the rays $t \mapsto(t, x+\mathcal{V} t)$ so,

$$
w_{0}(t, x, y)=\widetilde{w}_{0}(x-\mathcal{V} t, y), \quad \widetilde{w}_{0}(x, y)=w_{0}(0, x, y) .
$$

These lines moving at the group velocity are also called group lines.

The rays are parallel which leads for times $t=\mathcal{O}(1)$ to approximate solutions supported in the tube of rays with feet in the support of the initial data. As in the case of homogeneous equations in nonperiodic media, for times $t=\mathcal{O}(1 / \varepsilon)$ and linear phases, we prove that the support of the leading approximation extends beyond the tube of parallel rays. The spread of waves beyond this tube is described by a Schrödinger equation. This is called diffractive geometric optics (see [14], [7], [23], [17], [2]).

We next describe the diffractive geometric optics approximation for times $t=\mathcal{O}(1 / \varepsilon)$. In Section 4 we use an ansatz, similar to (1.11), but involving also a slow time, to describe Bloch wave packets exhibiting diffractive effects. Formal discussion of such effects can be found in the physical literature, for example in [32], [33]. In order to have infinite order expansions analogous to those for $t=\mathcal{O}(1)$ it is sufficient (and not far from necessary) that the $\mathcal{O}\left(\varepsilon^{2}\right)$ modulations of the coefficients satisfy the constraint,

$$
\left(\partial_{t}+\mathcal{V} . \partial_{x}\right)\left\{A_{1}(t, x, y), \rho_{1}(t, x, y)\right\}=0 \text {. }
$$

This very strong constraint is equivalent to the invariance of the modulations on the rays $(t, x+\mathcal{V} t)$. Define

$$
\gamma(t, x):=\int_{\mathbb{T}_{y}^{N}} \overline{\psi_{n}}(y)\left(\rho_{1}(t, x, y)(2 \pi i \omega)^{2}-\operatorname{div}_{y} A_{1}(t, x, y) \operatorname{grad}_{y}\right) \psi_{n}(y) d y .
$$

When (1.12) is satisfied, $\gamma(t, x)$ is constant on rays so,

$$
\gamma(t, x)=\widetilde{\gamma}(x-\mathcal{V} t), \quad \widetilde{\gamma}(x):=\gamma(0, x) .
$$

The leading term in the approximate solution is of the form

$$
e^{2 \pi i S / \varepsilon} \widetilde{a}(\varepsilon t, x-\mathcal{V} t) \psi_{n}\left(x / \varepsilon, \theta_{0}\right)
$$


where $\widetilde{a}(\mathcal{T}, x)$ satisfies the Schrödinger equation

$$
\left(4 \pi i \partial_{\mathcal{T}}-\nabla_{\theta}^{2} \omega\left(\partial_{x}, \partial_{x}\right)+\frac{\widetilde{\gamma}(x)}{\omega}\right) \widetilde{a}=0
$$

with the slow time variable $\mathcal{T}=\varepsilon t$ which is of order 1 when $t=\mathcal{O}(1 / \varepsilon)$. In our earlier paper [4] the approximation (1.14) is justified by weak convergence methods when (1.12) is satisfied. In the present paper we give sharp error estimates and infinitely accurate asymptotic expansions.

In addition, we prove that the same leading order term yields an approximation with relative error $\mathcal{O}\left(\varepsilon^{1-\beta}\right)$ under much milder conditions than (1.12). The conditions involve the average of $\gamma(t, x)$ along rays. It is reasonable that an observer moving on group lines will, over long times, be affected by the average of $\gamma(t, x)$ on the line. To start with, we suppose that the ray averages

$$
\widetilde{\gamma}(x):=\lim _{T \rightarrow+\infty} \frac{1}{T} \int_{0}^{T} \gamma(t, x+\mathcal{V} t) d t
$$

exist. This is equivalent to the fact that the solution of the transport equation

$$
\left(\partial_{t}+\mathcal{V} . \partial_{x}\right) g=\gamma(t, x)-\widetilde{\gamma}(x-\mathcal{V} t),
$$

is sublinear in time. We make the ray average hypothesis from Definition 5.2 which is much weaker than (1.12). There is a $0 \leq \beta<1$ so that for all $\alpha$, the solution $g_{\alpha}(t, x)$ of

$$
\left(\partial_{t}+\mathcal{V} . \partial_{x}\right) g_{\alpha}=\partial_{t, x}^{\alpha}(\gamma(t, x)-\widetilde{\gamma}(x-\mathcal{V} t)),\left.\quad g_{\alpha}\right|_{t=0}=0
$$

satisfies $(1+t)^{-\beta} g_{\alpha} \in L^{\infty}\left(\left[0, \infty\left[\times \mathbb{R}^{N}\right)\right.\right.$. This hypothesis is satisfied with $\beta=0$ if $\gamma$ is $(t, x)$-periodic with any period, and also for almost all quasiperiodic $\gamma$ and group velocities $\mathcal{V}$. It is proved with possibly positive $\beta$ for quite general smooth almost periodic $\gamma$ in $\S 5.1 .2$.

So far we have discussed the construction of approximate solutions. We next give a precise result for the initial value problem for the wave equation (1.1). Consider the oscillatory initial conditions

$$
u^{\varepsilon}(0, x)=b(x) e^{2 \pi i x \cdot \theta_{0} / \varepsilon} \psi_{n}\left(x / \varepsilon, \theta_{0}\right), \quad \partial_{t} u^{\varepsilon}(0, x)=\frac{c(x)}{\varepsilon} e^{2 \pi i x \cdot \theta_{0} / \varepsilon} \psi_{n}\left(x / \varepsilon, \theta_{0}\right),
$$

with $b, c \in \cap_{s \geq 0} H^{s}\left(\mathbb{R}^{N}\right)$. Denote by $\omega^{ \pm}$the two roots of $(1.6)$, by $S^{ \pm}=\omega^{ \pm} t+\theta_{0} x$ the corresponding phases, and by $\mathcal{V}^{ \pm}$the two group velocities. Define $\widetilde{w}_{0}^{ \pm}(\mathcal{T}, x, y):=$ $a^{ \pm}(\mathcal{T}, x) \psi_{n}\left(y, \theta_{0}\right)$ with the scalar valued $a^{ \pm}$determined by the Schrödinger equations

$$
\left(4 \pi i \partial_{\mathcal{T}} \mp \nabla_{\theta}^{2} \omega^{ \pm}\left(\partial_{x}, \partial_{x}\right)+\frac{\widetilde{\gamma}^{ \pm}(x)}{\omega^{ \pm}}\right) a^{ \pm}=0
$$

with initial data,

$$
\left.a^{+}\right|_{\mathcal{T}=0}=\frac{b(x)}{2}+\frac{c(x)}{4 \pi i \omega^{+}},\left.\quad a^{-}\right|_{\mathcal{T}=0}=\frac{b(x)}{2}-\frac{c(x)}{4 \pi i \omega^{-}}
$$


chosen so that the Cauchy data of $v^{\varepsilon}$ match those of $u^{\varepsilon}$ as well as possible (see $§ 5.3$ ). The following result follows from Theorem 5.13.

Theorem 1.3. Assume that $\widetilde{\gamma}$ satisfies the ray average hypothesis with parameter $0 \leq$ $\beta<1$ for both group velocities $\pm \mathcal{V}$, and that $\widetilde{w}_{0}^{ \pm}(\mathcal{T}, x, y)$ are defined as above. Define

$$
v^{\varepsilon}(t, x):=\sum_{ \pm} e^{2 \pi i S^{ \pm}(t, x) / \varepsilon} \widetilde{w}_{0}^{ \pm}(\varepsilon t, x \mp \mathcal{V} t, x / \varepsilon)
$$

then $v^{\varepsilon}$ is an approximate solution with relative error $\mathcal{O}\left(\varepsilon^{1-\beta}\right)$. Precisely, for any $T>0$ the error and its first order derivatives satisfy,

$$
\sup _{0 \leq t \leq T / \varepsilon} \sup _{|\alpha| \leq 1}\left\|\left(\varepsilon \partial_{t, x}\right)^{\alpha}\left(u^{\varepsilon}(t)-v^{\varepsilon}(t)\right)\right\|_{L^{2}\left(\mathbb{R}^{N}\right)} \leq C \varepsilon^{1-\beta},
$$

while the norms of $\left(\varepsilon \partial_{t, x}\right)^{\alpha} u^{\varepsilon}$ and $\left(\varepsilon \partial_{t, x}\right)^{\alpha} v^{\varepsilon}$ are $\mathcal{O}(1)$.

Remark 1.4. i. In the above theorem, as throughout this paper, $C$ denotes a constant that does not depend on $\varepsilon$. ii. The construction of the first corrector in the proof of Theorem 1.3 fails when the ray average hypothesis is not satisfied.

This result is surprising since one might expect that traversing $\mathcal{O}\left(1 / \varepsilon^{2}\right)$ periods of the background medium might destroy the wave packet structure. There are three countervailing influences;

i. The Bloch plane waves are solutions of the unperturbed equation for all time.

ii. The perturbations $\varepsilon^{2} \rho_{1}, \varepsilon^{2} A_{1}$ are scaled with $\varepsilon$ so that their effect is felt at times of order $1 / \varepsilon$.

iii. The term $\gamma(t, x)$ from the perturbations has well defined averages along rays, and, its integral along long segments of group lines differ little from the values predicted by the average.

The Schrödinger approximation of diffractive geometric optics comes from a second order approximation of the dispersion relation. The diffractive effect comes from the nonlinear character of the effective dispersion relation of periodic materials (see [13], [31] for other instances of this effect). The same is also true of the parabolic or paraxial approximation for waves propagating in a privileged direction (see [6], [28], [34]).

When the periodicity is on the atomic or even nano scale, it is impossible to perform numerical simulations of the differential equation to study propagations over macroscopic distances. The only hope is to replace the equations by others whose coefficients do not vary on the microscopic scale. The approximations of geometric optics and diffractive geometric optics produce such equations.

When the group velocity $\mathcal{V}$ is zero (which happens, at least, at the bottom and top of each Bloch band), the geometric optics scaling shows that Bloch wave packets (1.11) are 
essentially stationary for times $t \sim 1$. The diffractive scaling shows that this trapping persists for $t \sim 1 / \varepsilon$ when the modulations are $\mathcal{O}\left(\varepsilon^{2}\right)$ and satisfy the ray average hypothesis. Experimental exploitation of this phenomenon to slow light are cited above.

Furthermore, there exists no solution of the type (1.11) with temporal frequency $\omega$ when $4 \pi^{2} \omega^{2}$ is in a gap of the Bloch spectrum, i.e., when for all $n \geq 0$ and $\theta \in\left[0,1\left[^{N}\right.\right.$, $4 \pi^{2} \omega^{2} \neq \lambda_{n}(\theta)$. Arbitrary initial data are resolved into waves whose temporal frequencies never lie in these forbidden zones. An asymptotic analysis like that of $\S 3$, shows that when waves with forbidden frequencies arrive at the periodic medium from a medium which supports such frequencies, the waves are totally reflected. These properties are fundamental features of photonic crystals (see [26]).

The analysis of this paper is for scalar wave equations. The case of first order systems with elliptic spatial part is susceptible to an analogous analysis. There are complications from the vector nature and simplifications because the subtle $L^{2}$ estimates of $\S 5.2$ are not required. The case of Maxwell's equations poses additional difficulties as the spatial part is not elliptic. The compactness of resolvents holds on functions satisfying the $\varepsilon$ dependent and rapidly oscillatory divergence free condition $\operatorname{div}\left(\epsilon^{\varepsilon}(x) E\right)=0$ where $\epsilon^{\varepsilon}$ is a sequence of dielectric permittivities. This is the subject of a future project.

This paper is organized as follows. Section 2 recalls some facts about the Bloch spectral decomposition. In the case of purely periodic problems, with $\rho_{1}=0$ and $A_{1}=0$, the solutions have an exact representation using this decomposition. Infinite order asymptotics can be derived by performing an asymptotic analysis of the resulting integrals. These computations yield our principal results in a very special case. And, they motivate the ansatz for the geometric optics and diffractive geometric optics scales. They do not give a hint concerning the impact of the perturbations, $\rho_{1}, A_{1}$.

Section 3 presents the analysis at the scale $t \sim 1$ of geometric optics and for larger modulations $\varepsilon \rho_{1}, \varepsilon A_{1}$ instead of $\varepsilon^{2} \rho_{1}, \varepsilon^{2} A_{1}$. The construction of the approximations (1.11) introduces into the Bloch wave context projection techniques developed in nonlinear geometric optics, and, the fundamental identities of perturbation theory. The approximation solves the differential equation (1.1) with infinitely small residual. The standard energy estimate for $P^{\varepsilon}$ implies that the energy of the error is infinitely small. However, the operators $\left(P^{\varepsilon}\right)^{-1}$ are usually not uniformly bounded on higher Sobolev spaces. A nontrivial stability result in Section 3.4 shows that $\left(P^{\varepsilon}\right)^{-1}$ amplifies higher derivatives at most polynomially in $1 / \varepsilon$. Since the expansions have residuals that are $\mathcal{O}\left(\varepsilon^{\infty}\right)$, this suffices to show that high derivatives of the error are also $\mathcal{O}\left(\varepsilon^{\infty}\right)$. Though our main interest is in diffraction, these results on the scale $t=\mathcal{O}(1)$ are new. For these times scales the analysis could have been performed for nonlinear phases and their curved wave fronts. For the diffractive time scale, it is important that the phases are linear. 
Section 4 presents the analysis at the scale $t \sim 1 / \varepsilon$ of diffractive geometric optics when the modulations are constant on rays moving at the group velocity, that is satisfy (1.12). The hard new work is devoted to computing the profile equations which determine $w_{0}$ and the correctors $w_{j}$ in (1.11). Otherwise it follows the pattern of rigorous asymptotic analysis established in Section 3;

i. Construct profiles.

ii. Use Borel's theorem to construct the approximate solutions and estimate the residual.

iii. Use the stability estimate to prove accuracy.

Section 5 is devoted to producing leading order approximations when (1.12) is not satisfied but the ray average hypothesis is. In this case one does not achieve infinite order accuracy. We construct a three term expansion with residual $\mathcal{O}\left(\varepsilon^{2-\beta}\right)$. We use all of the preparatory work in the preceding sections. The gradient of the error is easily estimated by the standard energy estimate. A subtle stability argument is required to obtain $L^{2}$ estimates for the error. The oscillatory initial value problem is solved in Section 5.3 using two phases and correspondingly two approximate solutions. In Section 5.4 we show that the analysis for smooth envelopes is sufficient to yield strong convergence for the leading term asymptotics in diffractive geometric optics for envelopes which are only $H^{1}\left(\mathbb{R}^{N}\right)$.

Acknowledgements. The research of G. Allaire and M. Palombaro is partially supported by the Research Training Network MULTIMAT MRTN-CT-2004-505226 funded by the EEC. The research of G. Allaire is partially supported by the DEFI project at INRIA Saclay Ile de France and by the Chair "Mathematical modelling and numerical simulation, F-EADS - Ecole Polytechnique - INRIA". The research of J. Rauch is partially supported by the U.S. National Science Foundation under grant NSF-DMS-0104096. M. Palombaro and J. Rauch thank the CMAP at the École Polytechnique and its members for their hospitality.

\section{The PURELY PERIODIC CASE}

In this section we make the assumption that

$$
A_{1} \equiv 0 \quad \text { and } \quad \rho_{1} \equiv 0
$$

so the coefficients of (1.1) are periodic.

2.1. Bloch spectral decomposition. We briefly recall the Bloch decomposition. See $[9,8,12,25,29,37]$ for more details. Write each $\xi \in \mathbb{R}^{n}$ as $\mathbf{n}+\theta$ with $\mathbf{n} \in \mathbb{Z}^{N}$ and 
$\theta \in\left[0,1\left[^{N}\right.\right.$. Expressing $u(y)$ in terms of its Fourier transform, $\hat{u}(\xi)$, yields

$$
u(y)=\int_{[0,1[N} e^{2 \pi i \theta \cdot y}\left(\sum_{\mathbf{n} \in \mathbb{Z}^{N}} e^{2 \pi i \mathbf{n} \cdot y} \hat{u}(\theta+\mathbf{n})\right) d \theta .
$$

The function in parentheses is periodic with respect to $y$. The integrand is a function $g$ which is $\theta$-periodic in the sense that $y \rightarrow e^{-2 \pi i \theta . y} g(y)$ is periodic with period 1 in each $y_{j}$. This decomposes $L^{2}\left(\mathbb{R}^{N}\right)$ as the direct integral over $\theta$ of the Hilbert space of $\theta$-periodic functions. The parameter $\theta$ is called the Bloch frequency.

The partial derivatives of $\theta$-periodic functions are $\theta$-periodic and the product of a $\theta$-periodic function with a periodic function is $\theta$-periodic. Therefore, the differential operators $\operatorname{div}_{y} A_{0}(y) \operatorname{grad}_{y}$ and $P^{\varepsilon}$ (upon the change of variable $x=\varepsilon y$ ) map $\theta$-periodic functions to themselves. Therefore, the Bloch decomposition reduces these operators. Thus, arbitrary solutions of (1.1) are integrals over $\theta$ of $\theta$-periodic solutions.

To analyse the $\theta$-periodic solutions reason as follows. The unitary mapping $v \mapsto$ $e^{2 \pi i \theta . y} v$ on $L^{2}\left(\mathbb{T}^{N}\right)$ intertwines $\operatorname{div}_{y} A_{0} \operatorname{grad}_{y}$ with domain equal to the $\theta$-periodic elements of $H^{2}$ with the selfadjoint elliptic operator

$$
\mathbb{A}(\theta) \psi:=-\left(\operatorname{div}_{y}+2 i \pi \theta\right)\left(A_{0}(y)\left(\operatorname{grad}_{y}+2 i \pi \theta\right) \psi\right)
$$

with domain equal to periodic $H^{2}$.

Standard elliptic theory implies that for all $\theta$, the eigenvalue problem (1.7) has a countable nondecreasing sequence of real eigenvalues $\left\{\lambda_{n}(\theta)\right\}_{n \geq 1}$ repeated according to their multiplicity and $L^{2}\left(\mathbb{T}^{N}, \rho_{0}(y) d y\right)$ orthonormal eigenfunctions $\left\{\psi_{n}(y, \theta)\right\}_{n \geq 1}$, periodic in $y$ and depending measurably on $\theta$. The $\theta$-periodic functions are linear combinations in $n$ of the eigenfunctions

$$
e^{2 \pi i \theta \cdot y} \psi_{n}(y, \theta)
$$

The following lemma makes this precise.

Lemma 2.1. Let $u(y), v(y) \in L^{2}\left(\mathbb{R}^{N}\right)$. Define their Bloch coefficients for $n \geq 1$ and $\theta \in \mathbb{T}^{N}$

$$
\alpha_{n}(\theta):=\int_{\mathbb{R}^{N}} \rho_{0}(y) u(y) \bar{\psi}_{n}(y, \theta) e^{-2 i \pi \theta \cdot y} d y, \quad \beta_{n}(\theta):=\int_{\mathbb{R}^{N}} \rho_{0}(y) v(y) \bar{\psi}_{n}(y, \theta) e^{-2 i \pi \theta \cdot y} d y .
$$

Then, $\alpha_{n}, \beta_{n}$ belong to $L^{2}\left(\left[0,1\left[^{N}\right)\right.\right.$ and

$$
u(y)=\sum_{n \geq 1} \int_{\left[0,1\left[^{N}\right.\right.} \alpha_{n}(\theta) \psi_{n}(y, \theta) e^{2 i \pi \theta \cdot y} d \theta, \quad v(y)=\sum_{n \geq 1} \int_{\left[0,1\left[^{N}\right.\right.} \beta_{n}(\theta) \psi_{n}(y, \theta) e^{2 i \pi \theta \cdot y} d \theta,
$$

and they satisfy the Parseval equality

$$
\int_{\mathbb{R}^{N}} \rho_{0}(y) u(y) \bar{v}(y) d y=\sum_{n \geq 1} \int_{\left[0,1\left[^{N}\right.\right.} \alpha_{n}(\theta) \bar{\beta}_{n}(\theta) d \theta .
$$


The Bloch transform $u \rightarrow\left\{\alpha_{n}(\theta)\right\}_{n \geq 1}$ is an isometry from $L^{2}\left(\mathbb{R}^{N}\right)$ into $\ell^{2}\left(L^{2}\left(\left[0,1\left[^{N}\right)\right)\right.\right.$ that diagonalizes the elliptic operator in (1.1), in the sense that, for $u, v$ in $H^{1}\left(\mathbb{R}^{N}\right)$,

$$
\int_{\mathbb{R}^{N}} A_{0}(y) \nabla u(y) \cdot \nabla \bar{v}(y) d y=\sum_{n \geq 1} \int_{\left[0,1\left[^{N}\right.\right.} \lambda_{n}(\theta) \alpha_{n}(\theta) \bar{\beta}_{n}(\theta) d \theta .
$$

Arbitrary solutions of the wave equation (1.1), are linear combinations over $\theta$ and $n$ of the Bloch plane waves (1.5), (1.6). In this section we show that there exist spectrally localized solutions which have an asymptotic expansion whose leading term has the form of a Bloch wave with slowly varying amplitude, that is

$$
a(t, x) e^{2 \pi i(\omega t+\theta \cdot x) / \varepsilon} \psi_{n}(x / \varepsilon, \theta) .
$$

2.2. Explicit solutions. Throughout this paper we make the following assumption: for a fixed $\theta_{0} \in\left[0,1{ }^{N}\right.$ and integer $n \geq 1$,

$$
\lambda_{n}\left(\theta_{0}\right)>0 \text { is a simple eigenvalue. }
$$

Remark 2.2. Recall (see [8], [12], [25], [29]) that the minimum of $\lambda_{1}(\theta)$ is zero and is uniquely attained at $\theta=0$. This is a consequence of the maximum principle. The Hessian matrix at $\theta=0, \nabla_{\theta} \nabla_{\theta} \lambda_{1}(0)$ is equal to the usual homogenized matrix for equation (1.1). Therefore $\exists C>0$ such that $\lambda_{1}(\theta) \geq C|\theta|^{2}$. On the other hand, for $n \geq 2$, there exists a positive constant $C>0$ so that $\min _{\theta} \lambda_{n}(\theta) \geq C>0$.

Remark 2.2 implies that $\lambda_{n}\left(\theta_{0}\right)>0$ except if $n=1$ and $\theta_{0} \equiv 0 \bmod \mathbb{Z}^{N}$. The important part of assumption (2.2) is the simplicity of the eigenvalue. If the eigenvalue had multiplicity independent of $\theta$ on a neighborhood of $\theta_{0}$ an analogous analysis could be performed. We do not know any scalar examples of this kind. For systems such a generalization would be natural. In our scalar setting, simplicity is generic [1]. Since $\mathbb{A}(\theta)$ has compact resolvent and depends analytically on $\theta$, simplicity implies that the $n^{\text {th }}$ eigencouple of (1.7) is analytic in a neighborhood of $\theta_{0}$ (see e.g. [24]). Choose $\omega(\theta)$ an analytic solution of the dispersion relation (1.6) defined near $\theta_{0}$.

Under these conditions, if $a \in C_{0}^{\infty}\left(\mathbb{R}^{N}\right)$, then for $\varepsilon$ small the expressions

$$
u^{\varepsilon}(t, x):=\varepsilon^{-N} \int_{\left[0,1\left[{ }^{N}\right.\right.} \psi_{n}\left(\frac{x}{\varepsilon}, \theta\right) e^{2 \pi i \omega(\theta) t / \varepsilon} e^{2 \pi i x \cdot \theta / \varepsilon} a\left(\frac{\theta-\theta_{0}}{\varepsilon}\right) d \theta
$$

are superpositions of Bloch plane waves spectrally localized near $\theta_{0}$ so are exact solution of (1.1). (To treat the exceptional cases where one of the coordinates of $\theta_{0}$ vanishes, switch to a fundamental domain $\left[-c, 1-c\left[^{N}\right.\right.$ with $0<c<1$ such that $\theta_{0}$ lies in its interior.) Change variable letting $\zeta:=\left(\theta-\theta_{0}\right) / \varepsilon$ to find

$$
u^{\varepsilon}=e^{2 \pi i x \cdot \theta_{0} / \varepsilon} \int \psi_{n}\left(\frac{x}{\varepsilon}, \theta_{0}+\varepsilon \zeta\right) e^{2 \pi i t \omega\left(\theta_{0}+\varepsilon \zeta\right) / \varepsilon} e^{2 \pi i x . \zeta} a(\zeta) d \zeta,
$$

an expression prepared for Taylor expansion. 
2.3. The geometric optics time scale $\mathbf{t} \sim \mathbf{1}$. Recalling definition (1.9) of the group velocity $\mathcal{V}$, Taylor expansion in $\varepsilon$ of infinite and finite orders respectively yield,

$$
\begin{gathered}
\psi_{n}\left(y, \theta_{0}+\varepsilon \zeta\right) \sim \psi_{n}\left(y, \theta_{0}\right)+\sum_{j \geq 1} \varepsilon^{j} g_{j}(y, \zeta), \\
\omega\left(\theta_{0}+\varepsilon \zeta\right)=\omega\left(\theta_{0}\right)-\mathcal{V} \cdot \varepsilon \zeta+\varepsilon^{2} k(\varepsilon, \zeta) .
\end{gathered}
$$

Then,

$$
\begin{aligned}
e^{2 \pi i t \omega\left(\theta_{0}+\varepsilon \zeta\right) / \varepsilon} & =e^{2 \pi i t \omega\left(\theta_{0}\right) / \varepsilon} e^{-2 \pi i t \mathcal{V} \cdot \zeta} e^{2 \pi i(\varepsilon t) k(\varepsilon, \zeta)} \\
& =e^{2 \pi i t \omega\left(\theta_{0}\right) / \varepsilon} e^{-2 \pi i t \mathcal{V} \cdot \zeta}\left(1+\sum_{j \geq 1}(\varepsilon t)^{j} k_{j}(\varepsilon, \zeta)\right),
\end{aligned}
$$

where the last line uses a Taylor expansion of $s \mapsto e^{2 \pi i s k(\varepsilon, \zeta)}$ about $s=0$. Define

$$
v(x):=\int e^{2 \pi i x \cdot \zeta} a(\zeta) d \zeta
$$

Injecting (2.4) and (2.5) into (2.3) yields the expansion

$$
u^{\varepsilon} \sim e^{2 \pi i S / \varepsilon}\left(w_{0}(t, x, x / \varepsilon)+\varepsilon w_{1}(t, x, x / \varepsilon)+\cdots\right), \quad S:=\omega\left(\theta_{0}\right) t+x . \theta_{0},
$$

with leading term,

$$
w_{0}(t, x, y)=v(x-\mathcal{V} t) \psi_{n}\left(y, \theta_{0}\right)
$$

From this calculation we learn three things. First, rigidly translating waves at the group velocity is a reasonable approximation. Second, an infinite order expansion (2.6) is a reasonable ansatz to try in more complicated problems. Finally, in (2.5), the expansion parameter is $\varepsilon t$ so when $\varepsilon t$ is not small, the approximation is not appropriate. For the diffractive scale $\varepsilon t \sim 1$, one needs to modify the method.

2.4. The diffractive time scale $\mathbf{t} \sim \mathbf{1} / \varepsilon$. The modification is to take the next term in the Taylor expansion in the exponent. Denote by $q$ the symmetric quadratic expression

$$
q(\zeta, \zeta):=\sum_{i, j=1}^{N} \frac{\partial^{2} \omega\left(\theta_{0}\right)}{\partial \theta_{i} \partial \theta_{j}} \zeta_{i} \zeta_{j} .
$$

Then,

$$
\omega\left(\theta_{0}+\varepsilon \zeta\right)=\omega\left(\theta_{0}\right)-\varepsilon \mathcal{V} \cdot \zeta+\varepsilon^{2} q(\zeta, \zeta) / 2+\varepsilon^{3} \sum_{j \geq 0} \varepsilon^{j} \ell_{j}(\zeta)
$$

and,

$$
e^{2 \pi i \omega\left(\theta_{0}+\varepsilon \zeta\right) t / \varepsilon}=e^{2 \pi i \omega\left(\theta_{0}\right) t / \varepsilon} e^{-2 \pi i t \mathcal{V} \cdot \zeta} e^{2 \pi i \varepsilon t q(\zeta, \zeta) / 2} e^{2 \pi i \varepsilon(\varepsilon t) \sum_{j \geq 0} \varepsilon^{j} \ell_{j}(\zeta)} .
$$

If $(\varepsilon t)$ is bounded, expansion in $\varepsilon$ is justified in the last term. The exact solution has the form

$$
\begin{gathered}
e^{2 \pi i S / \varepsilon} \widetilde{W}(\varepsilon, \varepsilon t, x-\mathcal{V} t, x / \varepsilon), \quad S=\omega\left(\theta_{0}\right) t+\theta_{0} \cdot x \\
\widetilde{W}(\varepsilon, \mathcal{T}, x, y):=\int \psi_{n}\left(y, \theta_{0}+\varepsilon \zeta\right) e^{2 \pi i \mathcal{T} q(\zeta, \zeta) / 2} e^{2 \pi i \varepsilon \mathcal{T} \sum_{j \geq 0} \varepsilon^{j} \ell_{j}(\zeta)} e^{2 \pi i x . \zeta} a(\zeta) d \zeta .
\end{gathered}
$$


Taylor expansion in $\varepsilon$ yields

$$
e^{2 \pi i \varepsilon \mathcal{T} \sum_{j \geq 0} \varepsilon^{j} \ell_{j}(\zeta)}=\left(1+\sum_{j \geq 1} \varepsilon^{j} h_{j}(\mathcal{T}, \zeta)\right) .
$$

Injecting (2.4) and (2.8) in the definition of $\widetilde{W}$ shows that

$$
\widetilde{W}(\varepsilon, \mathcal{T}, x, y) \sim \sum_{j \geq 0} \varepsilon^{j} \widetilde{w}_{j}(\mathcal{T}, x, y),
$$

with

$$
\widetilde{w}_{0}(\mathcal{T}, x, y)=\psi_{n}\left(y, \theta_{0}\right) \int e^{2 \pi i \mathcal{T} q(\zeta, \zeta) / 2} e^{2 \pi i x \cdot \zeta} a(\zeta) d \zeta .
$$

This shows that the solution has an asymptotic expansion of the form

$$
e^{2 \pi i S / \varepsilon} \widetilde{W}(\varepsilon, \varepsilon t, x-\mathcal{V} t, x / \varepsilon),
$$

with $\widetilde{W}$ satisfying (2.9).

In our treatment of diffractive geometric optics in modulated media, we take a slightly more permissive ansatz

$$
e^{2 \pi i(\omega t+\theta . x) / \varepsilon}\left(w_{0}(\mathcal{T}, t, x, y)+\varepsilon w_{1}(\mathcal{T}, t, x, y)+\cdots\right) .
$$

with the idea that the added flexibility might be needed. Interestingly, it will follow that the expansion has the more restricted form found above.

The operator $q\left(\partial_{x}, \partial_{x}\right)$ applied to $\widetilde{w}_{0}$ from (2.10) inserts a factor $q(2 \pi i \zeta, 2 \pi i \zeta)=$ $-4 \pi^{2} q(\zeta, \zeta)$ inside the integrand. The operator $\partial_{\mathcal{T}}$ applied to $\widetilde{w}_{0}$ inserts a factor $\pi i q(\zeta, \zeta)$. Therefore $\widetilde{w}_{0}$ satisfies the Schrödinger equation,

$$
\left(4 \pi i \partial_{\mathcal{T}}-q\left(\partial_{x}, \partial_{x}\right)\right) \widetilde{w}_{0}=0 .
$$

We will derive this equation in an entirely different manner in Section 4.

In the case of the constant coefficient wave equation, $\omega$ is homogeneous of degree 1 in $\theta$ and $q$ has rank $N-1$. For the present problem $q$ may have rank $N$. When $q$ has rank $N$, the Schrödinger equation has more rapid dispersion of waves. For example, in dimension $N=1$ the constant coefficient wave equation is nondispersive, while a periodic one dimensional medium will typically be dispersive for waves whose wave length is comparable to the period.

\section{Bloch Wave packets on a modulated BaCkground and $t=\mathcal{O}(1)$}

3.1. The two scale construction. This section considers solutions of the wave equation

$$
P^{\varepsilon}\left(t, x, \partial_{t, x}\right) u^{\varepsilon}:=\rho^{\varepsilon} \frac{\partial^{2} u^{\varepsilon}}{\partial t^{2}}-\operatorname{div}_{x}\left(A^{\varepsilon} \operatorname{grad}_{x} u^{\varepsilon}\right)=0 .
$$

for times $t=\mathcal{O}(1)$. This time scale of ordinary geometric optics is an essential first step in treating the diffractive case. 
The coefficients are assumed to be of the form

$$
\rho^{\varepsilon}=\rho_{0}(x / \varepsilon)+\varepsilon \rho_{1}(t, x, x / \varepsilon), \quad A^{\varepsilon}=A_{0}(x / \varepsilon)+\varepsilon A_{1}(t, x, x / \varepsilon),
$$

where the $\rho_{j}, A_{j}$ satisfy (1.3), and, (1.4), from $\S 1$. For the geometric time scale, the modulations are taken to be $\mathcal{O}(\varepsilon)$ in place of $\mathcal{O}\left(\varepsilon^{2}\right)$ for the diffractive scale. After the next paragraph a crude estimate suggests why such perturbations are expected to influence the leading term asymptotics for times $t$ of order 1 .

Motivated by the case of smoothly varying media, and the special case of purely periodic media in the Section 2.3, the ansatz expected to be valid for times $t=\mathcal{O}(1)$ is of two scale WKB type,

$$
u^{\varepsilon}(t, x) \sim e^{2 i \pi S(t, x) / \varepsilon}\left(w_{0}\left(t, x, \frac{x}{\varepsilon}\right)+\varepsilon w_{1}\left(t, x, \frac{x}{\varepsilon}\right)+\cdots\right)
$$

where the $w_{j}(t, x, y)$ are periodic functions of $y$ with period one. Equivalently, the $w_{j}$ are functions on the unit torus $\mathbb{T}_{y}^{N}:=\mathbb{R}^{N} / \mathbb{Z}^{N}$.

The case when $S$ is a linear function of $(t, x)$ is our principal interest since it is in that case that the rays are parallel and one finds Schrödinger type equations at the diffractive scale $t=\mathcal{O}(1 / \varepsilon)$. Write

$$
S(t, x)=\omega t+\theta \cdot x, \quad(\omega, \theta) \in \mathbb{R}^{1+N} \backslash 0 .
$$

It suffices to consider $\theta \in\left[0,1^{N}\right.$. Other values can be converted to these by incorporating a periodic exponential, $e^{2 \pi i n . x}$, in the profiles $w_{j}$. Given $w_{j}$, Borel's Theorem allows us to choose smooth $W(\varepsilon, t, x, y)$, periodic in $y$ with Taylor series in $\varepsilon$,

$$
W(\varepsilon, t, x, y) \sim w_{0}(t, x, y)+\varepsilon w_{1}(t, x, y)+\cdots .
$$

Approximate solutions are defined by,

$$
v^{\varepsilon}(t, x):=e^{2 \pi i S(t, x) / \varepsilon} W\left(\varepsilon, t, x, \frac{x}{\varepsilon}\right) .
$$

Distinct choices of $W$ yield approximate solutions whose difference is infinitely small in the limit $\varepsilon \rightarrow 0$. We choose $w_{j}$ and then $W$ so that $P^{\varepsilon} v^{\varepsilon} \sim 0$ in the sense of Taylor series in $\varepsilon$ at $\varepsilon=0$. Toward that end, use the identities

$$
\begin{aligned}
& \partial_{t}\left(e^{2 \pi i S / \varepsilon} W(\varepsilon, t, x, y)\right)=e^{2 \pi i S / \varepsilon}\left(\frac{2 \pi i \omega}{\varepsilon}+\partial_{t}\right) W \\
& \partial_{x}\left(e^{2 \pi i S / \varepsilon} W(\varepsilon, t, x, y)\right)=e^{2 \pi i S / \varepsilon}\left(\frac{2 \pi i \theta}{\varepsilon}+\partial_{x}\right) W \\
& \left(\frac{\partial_{y}}{\varepsilon}\right)\left(e^{2 \pi i S / \varepsilon} W(\varepsilon, t, x, y)\right)=e^{2 \pi i S / \varepsilon}\left(\frac{\partial_{y}}{\varepsilon}\right) W
\end{aligned}
$$

to show that

$$
e^{-2 \pi i S / \varepsilon} P^{\varepsilon}\left(e^{2 \pi i S / \varepsilon} W(\varepsilon, t, x, x / \varepsilon)\right)=R(\varepsilon, t, x, x / \varepsilon),
$$


with

$$
\begin{aligned}
R(\varepsilon, t, x, y) & =e^{-2 \pi i S / \varepsilon}\left[\rho^{\varepsilon} \partial_{t}^{2}\right. \\
& \left.-\left(\operatorname{div}_{x}+\frac{\operatorname{div}_{y}}{\varepsilon}\right) A^{\varepsilon}\left(\operatorname{grad}_{x}+\frac{\operatorname{grad}_{y}}{\varepsilon}\right)\right]\left(e^{2 \pi i S / \varepsilon} W(\varepsilon, t, x, y)\right) \\
& =\left[\left(\rho_{0}+\varepsilon \rho_{1}\right)\left(\frac{2 \pi i \omega}{\varepsilon}+\partial_{t}\right)^{2}\right. \\
& \left.-\left(\frac{\operatorname{div}_{y}+2 \pi i \theta}{\varepsilon}+\operatorname{div}_{x}\right)\left(A_{0}+\varepsilon A_{1}\right)\left(\frac{\operatorname{grad}_{y}+2 \pi i \theta}{\varepsilon}+\operatorname{grad}_{x}\right)\right] W .
\end{aligned}
$$

Equation (3.4) implies that $R(\varepsilon, t, x, y)$ admits a Taylor series in $\varepsilon$ at $\varepsilon=0$ with uniquely determined $y$-periodic functions $r_{j}$ such that

$$
R(\varepsilon, t, x, y) \sim \sum_{j=-2}^{\infty} \varepsilon^{j} r_{j}(t, x, y) .
$$

Since one substitutes $y=x / \varepsilon$, it would suffice to satisfy $r_{j}=0$ on the subspace of $(x, y)$ with $x$ parallel to $y$. We achieve the more ambitious goal of choosing the $w_{j}$ so that $r_{j}=0$ everywhere.

3.2. The leading order term. Next analyse the cascade of equations,

$$
r_{j}=0, \quad j=-2,-1,0,1, \cdots .
$$

The operator in brackets on the right hand side of (3.4) is collected according to the powers $\varepsilon^{j}, j=-2,-1,0,1$. The leading two orders are

$$
\varepsilon^{-2} \mathbb{L}\left(\omega, \theta, y, \partial_{y}\right)+\varepsilon^{-1} \mathbb{M}\left(\omega, \theta, y, \partial_{t}, \partial_{x}, \partial_{y}\right),
$$

where

$$
\mathbb{L}\left(\omega, \theta, y, \partial_{y}\right):=-4 \pi^{2} \omega^{2} \rho_{0}-\left(\operatorname{div}_{y}+2 i \pi \theta\right) A_{0}(y)\left(\operatorname{grad}_{y}+2 i \pi \theta\right)
$$

and

$$
\begin{aligned}
\mathbb{M}\left(\omega, \theta, x, y, \partial_{t}, \partial_{x}, \partial_{y}\right):=\rho_{0} 4 \pi i \omega \partial_{t} & -\left(\left(2 \pi i \theta+\operatorname{div}_{y}\right) A_{0} \operatorname{grad}_{x}+\operatorname{div}_{x} A_{0}\left(2 \pi i \theta+\operatorname{grad}_{y}\right)\right) \\
& +\left(\rho_{1}(2 \pi i \omega)^{2}-\left(2 \pi i \theta+\operatorname{div}_{y}\right) A_{1}\left(2 \pi i \theta+\operatorname{grad}_{y}\right)\right) .
\end{aligned}
$$

The highest order term in the residual is

$$
r_{-2}=\mathbb{L}\left(\omega, \theta, y, \partial_{y}\right) w_{0} .
$$

It comes from the terms of order $\varepsilon^{-2}$ in the operator and the term of order $\varepsilon^{0}$ in $W$. In order that $r_{-2}=0$ have nontrivial solutions, it is necessary and sufficient that

$$
\operatorname{ker} \mathbb{L}\left(\omega, \theta, y, \partial_{y}\right) \neq\{0\} .
$$


According to Bloch wave theory, as described in section $2.1, \mathbb{L}\left(\omega, \theta, y, \partial_{y}\right)$ has a nontrivial kernel on periodic functions if and only $\omega$ and $\theta$ satisfy, for some integer $n$, the dispersion relation (1.6). Equation (1.6) is equivalent to the eikonal equation

$$
4 \pi^{2}\left(\partial_{t} S\right)^{2}=\lambda_{n}\left(\partial_{x} S\right)
$$

When $\lambda_{n}(\theta) \neq 0,(1.6)$ has two roots $\omega= \pm \sqrt{\lambda_{n}(\theta)} / 2 \pi$ and there are two distinct eikonal equations

$$
2 \pi \partial_{t} S= \pm \sqrt{\lambda_{n}\left(\partial_{x} S\right)}
$$

corresponding to the two roots $\omega$. Recall assumption $(2.2)$ that $\lambda_{n}\left(\theta_{0}\right) \neq 0$ is a simple eigenvalue. From now on we make this choice of $n$ and $\theta_{0}$ and $\omega$ is a solution of

$$
4 \pi^{2} \omega^{2}=\lambda_{n}\left(\theta_{0}\right) \text {. }
$$

Definition 3.1. Denote by $\mathbb{L}$, the self adjoint operator $\mathbb{L}\left(\omega, \theta_{0}, y, \partial_{y}\right)$ on $L^{2}\left(\mathbb{T}^{N} ; d y\right)$ with domain equal to the periodic functions in $H^{2}\left(\mathbb{T}_{y}^{N}\right)$. Denote by $\Pi$ the projection operator onto $\mathbb{K}:=\operatorname{ker} \mathbb{L}\left(\omega, \theta_{0}, y, \partial_{y}\right)$ along the image of $\mathbb{L}$. $\Pi$ is orthogonal with respect to the scalar product of $L^{2}\left(\mathbb{T}^{N} ; d y\right)$ and not with respect to the scalar product of $L^{2}\left(\mathbb{T}^{N} ; \rho_{0}(y) d y\right)$. Denote by $Q \in \operatorname{Hom}\left(H^{-1}\left(\mathbb{T}_{y}^{N}\right) ; H^{1}\left(\mathbb{T}_{y}^{N}\right)\right)$ the partial inverse of $\mathbb{L}$ defined by

$$
Q \Pi=\Pi Q=0, \quad Q \mathbb{L}=\mathbb{L} Q=I-\Pi .
$$

Choose $\psi_{n}(y):=\psi_{n}\left(y, \theta_{0}\right)$ an eigenfunction spanning $\operatorname{ker} \mathbb{L}\left(\omega, \theta_{0}, y, \partial_{y}\right)$ and normalized by (1.8).

The equation $r_{-2}=0$ is equivalent to $w_{0} \in \mathbb{K}=$ ker $\mathbb{L}$, that is

$$
\Pi w_{0}=w_{0} .
$$

Summary. Equations (3.10) and (3.11) are equivalent to $r_{-2}=0$.

Equation (3.11) is equivalent to the fact that for each $(t, x), w_{0}$ is a multiple of $\psi_{n}$,

$$
w_{0}(t, x, y)=v(t, x) \psi_{n}(y), \quad v^{\varepsilon}(t, x)=v(t, x) \psi_{n}(x / \varepsilon) e^{2 \pi i(\omega t+\theta \cdot x) / \varepsilon}+\cdots .
$$

Comparing with Bloch plane waves (1.5), (1.6), one sees that $v^{\varepsilon}$ is a Bloch wave packet. Our preferred perspective on (3.11) is to view $\mathbb{K}=$ ker $\mathbb{L}$ as a one dimensional vector space. Then $(t, x) \mapsto w_{0}(t, x, \cdot)$ is a mapping from $\mathbb{R}^{1+N}$ with values in $\mathbb{K}$.

Using the definitions of $\mathbb{L}$ and $\mathbb{M}$, the term $r_{-1}$ is given by,

$$
r_{-1}=\mathbb{L} w_{1}+\mathbb{M} w_{0}
$$

so $r_{-1}=0$ if and only if,

$$
\mathbb{L} w_{1}+\mathbb{M} w_{0}=0 .
$$

Equation (3.14) involves both $w_{0}$ and $w_{1}$. This is typical of multiscale expansions. Equations at a single order in $\varepsilon$ involve profiles from more than one order. 
The operator $\mathbb{L}$ is not surjective. The information about $w_{0}$ in (3.14) is that $\mathbb{M} w_{0} \in \operatorname{Rg} \mathbb{L}$ (Rg denotes the range). That information does not involve $w_{1}$. To extract this type of information, each equation $r_{j}=0$ with $j \geq-1$, is split into its part in ker $\mathbb{L}$ and its part in $\operatorname{Rg} \mathbb{L}$. In other words, it is written as the equivalent pair

$$
\Pi r_{j}=0, \quad Q r_{j}=0 .
$$

The systematic use of these projections and partial inverses, inspired by the work of Joly-Métivier-Rauch [22], [23] is an innovation of this article.

Using (3.13) and the relation $\Pi \mathbb{L}=0, \Pi r_{-1}=0$ yields an equation for $w_{0}$ alone,

$$
\Pi \mathbb{M} w_{0}=0 \text {. }
$$

Taking into account (3.11), this is equivalent to

$$
\Pi \mathbb{M} \Pi w_{0}=0 .
$$

Define $\gamma \in C^{\infty}\left(\mathbb{R}_{x}^{N}\right)$ by

$\gamma(t, x):=\int_{\mathbb{T}_{y}^{N}} \overline{\psi_{n}(y)}\left(\rho_{1}(t, x, y)(2 \pi i \omega)^{2}-\left(2 \pi i \theta+\operatorname{div}_{y}\right) A_{1}(t, x, y)\left(2 \pi i \theta+\operatorname{grad}_{y}\right)\right) \psi_{n}(y) d y$.

Proposition 3.2. For any $w(t, x, y) \in C^{\infty}$,

$$
\Pi \mathbb{M} \Pi w=\left(4 \pi i \omega \partial_{t}-\sum_{j=1}^{N} 4 \pi i \omega \frac{\partial \omega}{\partial \theta_{j}}\left(\theta_{0}\right) \frac{\partial}{\partial x_{j}}+\gamma(t, x)\right) \Pi w
$$

Proof. From the definition of $\Pi$ and $\mathbb{M}$ one automatically has for arbitrary $w$,

$$
\Pi \mathbb{M} \Pi w=\left(i a_{0} \partial_{t}+\sum_{j=1}^{N} i a_{j} \frac{\partial}{\partial x_{j}}+c(t, x)\right) \Pi w,
$$

with constants $a_{\mu}$ and a zero order term $c(t, x)$. It suffices to compute the values of the coefficients. This is done by computing the differential operator on the test functions $\psi_{n}$, $t \psi_{n}$, and $x_{j} \psi_{n}$ to find,

$$
c \psi_{n}(y)=\Pi \mathbb{M} \Pi \psi_{n}(y), \quad i a_{0} \psi_{n}=\Pi\left(4 \pi i \omega \rho_{0}(y) \psi_{n}\right), \quad i a_{j} \psi_{n}=\left(\Pi \mathbb{M}\left(x_{j} \psi_{n}\right)\right)_{x_{j}=0} .
$$

The first relation shows that $c=\gamma$ using the formula for $\mathbb{M}$ and the definition of $\gamma$. The normalization (1.8) of $\psi_{n}$ together with the formula for $i a_{0} \psi_{n}$ yields

$$
a_{0}=4 \pi \omega .
$$

Injecting the definition of $\mathbb{M}$ yields

$$
i a_{j} \psi_{n}=-\Pi\left(\left(2 \pi i \theta+\operatorname{div}_{y}\right)\left(A_{0}(y) \mathbf{e}_{j} \psi_{n}\right)+\mathbf{e}_{j} A_{0}(y)\left(2 \pi i \theta+\operatorname{grad}_{y}\right) \psi_{n}\right) \text {. }
$$


The identification of $a_{j}$ requires first order perturbation theory as in (3.22) of the next proposition. Second order perturbation theory as in (3.23) is needed for diffractive geometric optics.

Proposition 3.3. Suppose that $\theta_{0}, \lambda_{n}\left(\theta_{0}\right)$, and $\omega$ are as above. Suppose that the coefficients $\rho, A$ and $\theta$ depend smoothly on a parameter $\alpha$ with their unperturbed values attained at $\alpha_{0}$ Then, there is a uniquely determined smooth simple eigenvalue $\lambda_{n}(\alpha)$, root $\omega(\alpha)$ and orthogonal projection $\Pi(\alpha)$ with their unperturbed values at $\alpha_{0}$. With' denoting differentiation with respect to $\alpha$, the following perturbation formulas hold,

$$
\Pi \mathbb{L}^{\prime} \Pi=0,
$$

and

$$
\Pi \mathbb{L}^{\prime \prime} \Pi-2 \Pi \mathbb{L}^{\prime} Q \mathbb{L}^{\prime} \Pi=0
$$

Proof. The equations defining $\Pi(\alpha)$ are,

$$
\Pi=\Pi^{*}, \quad \Pi^{2}=\Pi, \quad \mathbb{L} \Pi=0 .
$$

Since $\mathbb{L}$ is selfadjoint,

$$
\Pi \mathbb{L}=0
$$

Differentiate the last equation in (3.24) to find

$$
\mathbb{L} \Pi^{\prime}+\mathbb{L}^{\prime} \Pi=0 .
$$

Multiply by $\Pi$ on the left and use (3.25) to prove (3.22). Multiply (3.26) by $Q$ to find,

$$
(I-\Pi) \Pi^{\prime}=-Q \mathbb{L}^{\prime} \Pi \text {. }
$$

Differentiate (3.26) to find

$$
\mathbb{L} \Pi^{\prime \prime}+2 \mathbb{L}^{\prime} \Pi^{\prime}+\mathbb{L}^{\prime \prime} \Pi=0 .
$$

Multiply by $\Pi$ on the left, and use $\Pi^{\prime}=\Pi \Pi^{\prime}+(I-\Pi) \Pi^{\prime}$, to find

$$
2 \Pi \mathbb{L}^{\prime}\left(\Pi \Pi^{\prime}+(I-\Pi) \Pi^{\prime}\right)+\Pi \mathbb{L}^{\prime \prime} \Pi=0 .
$$

Use (3.22) and (3.27) to find (3.23), completing the proof of Proposition 3.3.

Returning to the formula for $a_{j}$, use (3.22) with $\alpha$ equal to the $j^{\text {th }}$ component of $\theta$. Then, prime denotes $\partial / \partial \theta_{j}$, so,

$$
\mathbb{L}^{\prime}=-8 \pi^{2} \rho_{0} \omega \frac{\partial \omega}{\partial \theta_{j}}-2 \pi i \mathbf{e}_{j} A_{0}\left(\operatorname{grad}_{y}+2 \pi i \theta\right)-\left(\operatorname{div}_{y}+2 \pi i \theta\right) A_{0} 2 \pi i \mathbf{e}_{j}
$$


where $\left\{\mathbf{e}_{j}\right\}_{j=1}^{N}$ is the standard basis for $\mathbb{R}_{\theta}^{N}$. Dividing this equation by $2 \pi$ and combining with (3.22), it follows that

$$
\Pi\left[-4 \pi \rho_{0} \omega \frac{\partial \omega}{\partial \theta_{j}}-i \mathbf{e}_{j} A_{0}\left(\operatorname{grad}_{y}+2 \pi i \theta\right)-\left(\operatorname{div}_{y}+2 \pi i \theta\right) A_{0} i \mathbf{e}_{j}\right] \Pi=0 .
$$

Apply this identity to $\psi_{n}$ and use (3.11) and (3.21) to find

$$
a_{j} \psi_{n}=-\Pi\left(4 \pi \rho_{0} \omega \frac{\partial \omega}{\partial \theta_{j}} \psi_{n}\right)=-4 \pi \omega \frac{\partial \omega}{\partial \theta_{j}} \Pi\left(\rho_{0} \psi_{n}\right) .
$$

The normalization (1.8) of $\psi_{n}$ completes the proof of the Proposition 3.2.

Summary. If $\theta_{0}, \omega$ are chosen to satisfy the dispersion relation (3.10) at a simple eigenvalue $\lambda_{n}\left(\theta_{0}\right), \mathcal{V}:=-\nabla_{\theta} \omega\left(\theta_{0}\right)$, and $w_{0}$ is determined from its initial data which is an arbitrary smooth function with values in $\mathbb{K}$ as the solution of the transport equation

$$
\left(\partial_{t}+\mathcal{V} . \partial_{x}\right) w_{0}+\frac{\gamma(t, x)}{4 \pi i} w_{0}=0
$$

then (and only then) $r_{-2}=0$ and $\Pi r_{-1}=0$.

3.3. Determination of the correctors. Inductively suppose that $J \geq 0$ and the smooth profiles $w_{j}, 0 \leq j \leq J$, have been determined so that $r_{-2}, r_{-1}, \ldots r_{J-2}$ and $\Pi r_{J-1}$ vanish. We show that the equations $Q r_{J-1}=0$ and $\Pi r_{J}=0$ yield a unique determination of $w_{J+1}$ from arbitrary initial data $\left.\Pi w_{J+1}\right|_{t=0}$. One has

$$
r_{J-1}=\mathbb{L} w_{J+1}+\mathbb{M} w_{J}+F_{J-1}\left(w_{0}, w_{1}, \ldots w_{J-1}\right),
$$

and

$$
r_{J}=\mathbb{L} w_{J+2}+\mathbb{M} w_{J+1}+F_{J}\left(w_{0}, w_{1}, \ldots w_{J}\right),
$$

where the last terms are smooth linear functionals of the profiles indicated.

The definition of $Q$ shows that the equation $Q r_{J-1}=0$ is equivalent to

$$
(I-\Pi) w_{J+1}=-Q\left(\mathbb{M} w_{J}+F_{J-1}\left(w_{0}, w_{1}, \ldots w_{J-1}\right)\right) .
$$

This determines the left hand side in terms of already known profiles.

The equation $\Pi r_{J}=0$ is equivalent to

$$
\Pi\left(\mathbb{M} w_{J+1}+F_{J}\left(w_{0}, w_{1}, \ldots w_{J}\right)\right)=0 .
$$

Using (3.29) write,

$$
w_{J+1}=\Pi w_{J+1}-Q\left(\mathbb{M} w_{J}+F_{J-1}\left(w_{0}, w_{1}, \ldots w_{J-1}\right)\right) .
$$

Plug this into (3.30) to find

$$
\Pi \mathbb{M} \Pi\left(\Pi w_{J+1}\right)+\Pi G_{J+1}\left(w_{0}, \ldots, w_{J}\right)=0 .
$$


Thanks to Proposition 3.2, this is a simple transport equation which determines $\Pi w_{J+1}$ from its arbitrary smooth initial data at $t=0$ and the previous profiles $\left(w_{0}, w_{1}, \ldots w_{J}\right)$. The transport is at the group velocity $\mathcal{V}=-\nabla_{\theta} \omega\left(\theta_{0}\right)$.

These computations prove the following theorem.

Theorem 3.4. Suppose that $\theta_{0}$ and $\omega$ satisfy the dispersion relation (3.10) at a simple eigenvalue $\lambda_{n}\left(\theta_{0}\right) \neq 0$. Given smooth $\mathbb{K}$ valued functions $g_{j}(x)$, there are uniquely determined smooth profiles $w_{j}(t, x, y)$ periodic in $y$ so that

$$
\left.w_{0}\right|_{t=0}=g_{0},\left.\quad \Pi w_{j}\right|_{t=0}=g_{j}, \quad \text { and } \quad \forall j, t, x, y, \quad r_{j}(t, x, y)=0 .
$$

The value of $w_{J}$ at $(t, x)$ is influenced only by the data $g_{0}, \ldots g_{J}$ at $x-\mathcal{V} t$. The leading term $w_{0}$ is $\mathbb{K}$ valued and is determined from (3.28).

The next result shows that once the profiles are constructed as above they serve to construct an infinitely accurate approximate solution in the sense that the residual and all of its derivatives are infinitely small as $\varepsilon \rightarrow 0$.

Theorem 3.5. Suppose that the $g_{j}$ have supports in a fixed compact set and the $w_{j}$ are as in the preceding theorem. Suppose that $W(\varepsilon, t, x, y) \in C^{\infty}\left(\left[0,1\left[\times\left[0, \infty\left[\times \mathbb{R}^{N} \times \mathbb{T}^{N}\right)\right.\right.\right.\right.$ with support over the tube of rays with feet in the support of the $g_{j}$ has Taylor expansion in $\varepsilon$,

$$
W(\varepsilon, t, x, y) \sim \sum_{j=0}^{\infty} \varepsilon^{j} w_{j}(t, x, y) .
$$

Define

$$
v^{\varepsilon}(t, x):=e^{2 \pi i S / \varepsilon} W(\varepsilon, t, x, x / \varepsilon) .
$$

Then $P^{\varepsilon} v^{\varepsilon}=\mathcal{O}\left(\varepsilon^{\infty}\right)$ in the sense that for any $T>0, \alpha \in \mathbb{N}^{1+N}$, and $n \in \mathbb{N}$, there is a $C$ so that

$$
\left\|\partial_{t, x}^{\alpha} P^{\varepsilon} v^{\varepsilon}\right\|_{L^{\infty}\left([0, T] \times \mathbb{R}^{N}\right)} \leq C \varepsilon^{n} .
$$

Remark 3.6. In Theorem 3.5 the assumption of compact support for the initial data $g_{j}$ can be replaced by a suitable uniform control of the derivatives at infinity.

Proof. The residual $R(\varepsilon, t, x, y)$, defined by (3.4), admits a Taylor expansion with terms $r_{j}$ which, by construction are identically equal to zero. The approximate solution, $v^{\varepsilon}$ satisfies

$$
P^{\varepsilon} v^{\varepsilon}=e^{2 \pi i S / \varepsilon} R(\varepsilon, t, x, x / \varepsilon) .
$$

By construction, $R \in C^{\infty}\left(\left[0,1\left[{ }_{\varepsilon} \times\left[0, \infty\left[{ }_{t} \times \mathbb{R}_{x}^{N} \times \mathbb{T}_{y}^{N}\right)\right.\right.\right.\right.$ with compact support in $x$ and each of its partial derivatives is infinitely flat at $\varepsilon=0$ uniformly on compact subsets of $(t, x, y)$. The result follows from Taylor's theorem. 
3.4. Stability. The stability estimate of this section implies that exact solutions are infinitely close to the approximate solution. If $u^{\varepsilon}$ is the exact solution with the same Cauchy data as $v^{\varepsilon}$ then $P^{\varepsilon}\left(u^{\varepsilon}-v^{\varepsilon}\right)=\mathcal{O}\left(\varepsilon^{\infty}\right)$ so the error is given by

$$
u^{\varepsilon}-v^{\varepsilon}=\left(P^{\varepsilon}\right)^{-1}\left(\mathcal{O}\left(\varepsilon^{\infty}\right)\right) .
$$

One needs estimates for $\left(P^{\varepsilon}\right)^{-1}$ which grow at worst polynomially in $1 / \varepsilon$ as $\varepsilon \rightarrow 0$. As a map from $L^{1}\left([0, T] ; L^{2}\left(\mathbb{R}^{N}\right)\right)$ to $C\left([0, T] ; H^{1}\left(\mathbb{R}^{N}\right)\right) \cap C^{1}\left([0, T] ; L^{2}\left(\mathbb{R}^{N}\right)\right)$ such an estimate is immediate from the energy identity. We prove that the $L^{2}\left(\mathbb{R}^{N}\right)$ norm of partial derivatives of order $s>1$ has growth no faster than $1 / \varepsilon^{k(s)}$ on time intervals of length of order $1 / \varepsilon$.

The proof is subtle since one cannot simply differentiate the equation. Taking a partial derivative $\partial$ of $P^{\varepsilon} u=0$ yields

$$
P^{\varepsilon}(\partial u)=\left[P^{\varepsilon}, \partial\right] u
$$

The commutator is a family, indexed by $\varepsilon$, of partial differential operators of degree 2 . The leading terms in the commutator come from the commutator of $\partial$ with the unperturbed operator. The unperturbed operator has coefficients which vary rapidly with $x$ but the coefficients do not vary rapidly in time. One finds that in shorthand $P^{\varepsilon} \partial_{x} u=\mathcal{O}(1 / \varepsilon) \partial^{2} u$ while $P^{\varepsilon} \partial_{t} u=\mathcal{O}(1) \partial^{2} u$. Injecting the first in a Gronwall argument yields growth in time like $e^{c t / \varepsilon}$. Differentiating the equation with respect to $x$ yields estimates which grow too fast.

The strategy is to differentiate with respect to $t$ only. The missing $x$ derivatives are recovered by an ellipticity argument. Control of $\partial_{t, x}^{\alpha} P^{\varepsilon} u$ and $\partial_{t}^{j} u$ in $L^{2}$ suffices to control all derivatives. The proof uses estimates associated to the uniformly elliptic family of operators $G^{\varepsilon}(t):=\operatorname{div} A^{\varepsilon} \operatorname{grad}, 0<\varepsilon \leq \varepsilon_{0}, t \in \mathbb{R}$.

Lemma 3.7. For all $1 \leq s \in \mathbb{N}$, there are constants $C=C(s)$ and $m=m(s) \geq 0$ so that for all $\left.t \in \mathbb{R}, \varepsilon \in] 0, \varepsilon_{0}\right], \phi \in H^{s}\left(\mathbb{R}^{n}\right)$,

$$
\|\phi\|_{H^{s}\left(\mathbb{R}^{N}\right)} \leq C\left(\left\|G^{\varepsilon}(t) \phi\right\|_{H^{s-2}\left(\mathbb{R}^{N}\right)}+\frac{1}{\varepsilon^{m}}\|\phi\|_{H^{s-1}\left(\mathbb{R}^{N}\right)}\right) .
$$

Proof. The proof is by induction on $s$. The case $s=1$ is true with $m=0$. This is an immediate consequence of the uniform elliptic estimate,

$$
\begin{aligned}
\left\|\partial_{x} \phi\right\|_{L^{2}\left(\mathbb{R}^{N}\right)}^{2} & \leq C \int_{\mathbb{R}^{N}}\left\langle G^{\varepsilon} \phi, \phi\right\rangle d x \leq C\|\phi\|_{H^{1}\left(\mathbb{R}^{N}\right)}\left\|G^{\varepsilon} \phi\right\|_{H^{-1}\left(\mathbb{R}^{N}\right)} \\
& \leq \frac{1}{2}\left(\left\|\partial_{x} \phi\right\|_{L^{2}\left(\mathbb{R}^{N}\right)}^{2}+\|\phi\|_{L^{2}\left(\mathbb{R}^{N}\right)}^{2}\right)+C\left\|G^{\varepsilon} \phi\right\|_{H^{-1}\left(\mathbb{R}^{N}\right)}^{2} .
\end{aligned}
$$

It follows that for all $\varepsilon, t$,

$$
\|\phi\|_{H^{1}\left(\mathbb{R}^{N}\right)} \leq C\left(\left\|G^{\varepsilon} \phi\right\|_{H^{-1}\left(\mathbb{R}^{N}\right)}+\|\phi\|_{L^{2}\left(\mathbb{R}^{N}\right)}\right) .
$$


Suppose that (3.31) is proved for $s \geq 1$. We derive the case $s+1$. It suffices to estimate the $L^{2}$ norm of $\partial_{x}^{\alpha} \phi$ when $|\alpha|=s+1$. Choose multiindices $\alpha^{\prime}$ and $\beta$ with $\alpha=\alpha^{\prime}+\beta$ and $\left|\alpha^{\prime}\right|=s$. Then

$$
\left\|\partial_{x}^{\alpha} \phi\right\|_{L^{2}\left(\mathbb{R}^{N}\right)} \leq\left\|\partial_{x}^{\beta} \phi\right\|_{H^{s}\left(\mathbb{R}^{N}\right)} \leq C\left(\left\|G^{\varepsilon} \partial_{x}^{\beta} \phi\right\|_{H^{s-2}\left(\mathbb{R}^{N}\right)}+\frac{1}{\varepsilon^{m(s)}}\left\|\partial_{x}^{\beta} \phi\right\|_{H^{s-1}\left(\mathbb{R}^{N}\right)}\right)
$$

using (3.31). Write

$$
G^{\varepsilon} \partial_{x}^{\beta} \phi=\partial_{x}^{\beta} G^{\varepsilon} \phi+\left[\partial_{x}^{\beta}, G^{\varepsilon}\right] \phi
$$

Since $|\beta| \leq 1$, the commutator is a differential operator of order 2 . The coefficients and their partial derivatives grow at most as $1 / \varepsilon^{2}$. Therefore,

$$
\left\|G^{\varepsilon} \partial_{x}^{\beta} \phi\right\|_{H^{s-2}\left(\mathbb{R}^{N}\right)} \leq\left\|G^{\varepsilon} \phi\right\|_{H^{s-1}\left(\mathbb{R}^{N}\right)}+\frac{C}{\varepsilon^{2}}\|\phi\|_{H^{s}\left(\mathbb{R}^{N}\right)}
$$

Together with (3.32), this completes the induction.

The next important stability proposition is stated only in the case of infinitely small source terms. It is equivalent to an estimate for $\left(P^{\varepsilon}\right)^{-1}$ which grows polynomially in $1 / \varepsilon$. The result proves stability on time intervals of length $\mathcal{O}(1 / \varepsilon)$. The long intervals are needed for the diffractive case.

Proposition 3.8. Suppose that $T>0$, and $v^{\varepsilon} \in C^{\infty}\left([0, T / \varepsilon] \times \mathbb{R}^{N}\right)$ satisfies $P^{\varepsilon} v^{\varepsilon}=$ $\mathcal{O}\left(\varepsilon^{\infty}\right)$ with Cauchy data $\mathcal{O}\left(\varepsilon^{\infty}\right)$ in the sense that, for all $\alpha, \beta, k$, there is a constant $C=C(k, \alpha, \beta)$ so that

$$
\left\|\left.\partial_{x}^{\beta}\left\{v^{\varepsilon}, \partial_{t} v^{\varepsilon}\right\}\right|_{t=0}\right\|_{L^{2}\left(\mathbb{R}^{N}\right)}+\sup _{0 \leq t \leq T / \varepsilon}\left\|\partial_{t, x}^{\alpha} P^{\varepsilon} v^{\varepsilon}(t)\right\|_{L^{2}\left(\mathbb{R}^{N}\right)} \leq C \varepsilon^{k} .
$$

Then, $v^{\varepsilon}$ is infinitely small in the sense that for any $\alpha, k$ there is another constant $C=$ $C(\alpha, k, T)$ so that

$$
\sup _{0 \leq t \leq T / \varepsilon}\left\|\partial_{t, x}^{\alpha} v^{\varepsilon}(t)\right\|_{L^{2}\left(\mathbb{R}^{N}\right)} \leq C \varepsilon^{k}
$$

Proof. The first step is to show that $\left\|\partial_{t}^{j} \partial_{x}^{\beta} v^{\varepsilon}(0)\right\|_{L^{2}\left(\mathbb{R}^{N}\right)}=\mathcal{O}\left(\varepsilon^{\infty}\right)$. For $j \leq 2$ this is implied by (3.33). The proof is by induction on $j$. Suppose the assertion is proved for indices $\leq j$. We prove the case $j+1$. Use the relation

$$
P^{\varepsilon} \partial_{t}^{j-1} \partial_{x}^{\beta} v^{\varepsilon}=\partial_{t}^{j-1} \partial_{x}^{\beta} P^{\varepsilon} v^{\varepsilon}+\left[P^{\varepsilon}, \partial_{t}^{j-1} \partial_{x}^{\beta}\right] v^{\varepsilon}=\mathcal{O}\left(\varepsilon^{\infty}\right)+\left[P^{\varepsilon}, \partial_{t}^{j-1} \partial_{x}^{\beta}\right] v^{\varepsilon} .
$$

Dividing by $\rho$, this expresses the derivative $\partial_{t}^{j+1} \partial_{x}^{\beta} v^{\varepsilon}$ at time $t=0$ as a sum of terms which are $\mathcal{O}\left(\varepsilon^{\infty}\right)$ by the inductive hypothesis.

Estimate (3.34) is proved for $|\alpha| \leq n$ by induction on $n$. The case $n=1$ uses the standard energy method. Considering the real and imaginary parts of $v^{\varepsilon}$, it suffices to 
consider real solutions. Suppress the $\varepsilon$ dependence of $v$. Multiply $P^{\varepsilon} v$ by $\partial_{t} v$ and integrate in space to find,

$$
\begin{gathered}
\partial_{t}\left(\frac{1}{2} \int_{\mathbb{R}^{N}}\left(\rho^{\varepsilon}\left(\partial_{t} v\right)^{2}+\left\langle A^{\varepsilon} \operatorname{grad} v, \operatorname{grad} v\right\rangle\right) d x\right)= \\
\int_{\mathbb{R}^{N}} \partial_{t} v(t) P^{\varepsilon} v(t) d x+\frac{\varepsilon}{2} \int_{\mathbb{R}^{N}}\left(\partial_{t} \rho_{1}\left(\partial_{t} v\right)^{2}+\left\langle\partial_{t} A_{1} \operatorname{grad} v, \operatorname{grad} v\right\rangle\right) d x
\end{gathered}
$$

For the quantity

$$
E(t):=\left(\frac{1}{2} \int_{\mathbb{R}^{N}}\left(\rho^{\varepsilon}\left(\partial_{t} v\right)^{2}+\left\langle A^{\varepsilon} \operatorname{grad} v, \operatorname{grad} v\right\rangle\right) d x\right)^{1 / 2}
$$

equivalent to the norm $\left\|\partial_{t, x} v(t)\right\|_{L^{2}\left(\mathbb{R}^{N}\right)}$ this shows that

$$
\frac{d}{d t} E^{2}(t) \leq C E(t)\left\|P^{\varepsilon} v(t)\right\|_{L^{2}\left(\mathbb{R}^{N}\right)}+C \varepsilon E^{2}(t) .
$$

Gronwall's method implies that there is a constant independent of $v, \varepsilon, t$ so that

$$
\left\|\partial_{t, x} v(t)\right\|_{L^{2}\left(\mathbb{R}^{N}\right)} \leq C e^{C \varepsilon t}\left\|\partial_{t, x} v(0)\right\|_{L^{2}\left(\mathbb{R}^{N}\right)}+C \int_{0}^{t} e^{C \varepsilon(t-s)}\left\|P^{\varepsilon} v(s)\right\|_{L^{2}\left(\mathbb{R}^{N}\right)} d s .
$$

Using assumption (3.33) and the fact that $\varepsilon t$ is bounded, this implies the case $|\alpha|=1$ of (3.34). Estimate $L^{2}\left(\mathbb{R}^{N}\right)$ norms as

$$
\begin{aligned}
\|v(t)\|_{L^{2}\left(\mathbb{R}^{N}\right)}=\left\|v(0)+\int_{0}^{t} \partial_{t} v(s) d s\right\|_{L^{2}\left(\mathbb{R}^{N}\right)} & \leq \mathcal{O}\left(\varepsilon^{\infty}\right)+\int_{0}^{t}\left\|\partial_{t} v(s)\right\|_{L^{2}\left(\mathbb{R}^{N}\right)} d s \\
& \leq \mathcal{O}\left(\varepsilon^{\infty}\right)+t \mathcal{O}\left(\varepsilon^{\infty}\right) .
\end{aligned}
$$

The last estimate uses the case $|\alpha|=1$. Since $t=\mathcal{O}(1 / \varepsilon)$ it follows that $\|v(t)\|_{L^{2}}=\mathcal{O}\left(\varepsilon^{\infty}\right)$ proving the case $|\alpha|=0$. This proves the $n=1$ case of the induction.

Suppose next that the result is known for $|\alpha| \leq n$. We prove the case $n+1$. The idea is to use (3.35) for the function $\partial_{t}^{n} v$. Toward that end compute

$$
P^{\varepsilon} \partial_{t}^{n} v=\partial_{t}^{n} P^{\varepsilon} v+\left[P^{\varepsilon}, \partial_{t}^{n}\right] v=\left[P^{\varepsilon}, \partial_{t}^{n}\right] v+\mathcal{O}\left(\varepsilon^{\infty}\right) .
$$

The commutator is a differential operator of degree $n+1$. The derivatives that appear are at most of order 2 in $x$. The coefficients are time derivatives of the coefficients of $P^{\varepsilon}$ so are $\mathcal{O}(\varepsilon)$. By induction the terms on the right of (3.36) involving derivatives of order $\leq n$ are $\mathcal{O}\left(\varepsilon^{\infty}\right)$. Therefore,

$$
\left\|\left[P^{\varepsilon}, \partial_{t}^{n}\right] v\right\|_{L^{2}\left(\mathbb{R}^{N}\right)} \leq C \varepsilon \sum_{\substack{j+|\beta|=n+1 \\|\beta| \leq 2}}\left\|\partial_{x}^{\beta} \partial_{t}^{j} v\right\|_{L^{2}\left(\mathbb{R}^{N}\right)}+\mathcal{O}\left(\varepsilon^{\infty}\right) .
$$

Applying (3.35) to $\partial_{t}^{n} v$ yields, for $\varepsilon t \leq T$,

$$
\left\|\partial_{t, x} \partial_{t}^{n} v(t)\right\|_{L^{2}} \leq \mathcal{O}\left(\varepsilon^{\infty}\right)+\int_{0}^{t} C \varepsilon \sum_{\substack{j+|\beta|=n+1 \\|\beta| \leq 2}}\left\|\partial_{x}^{\beta} \partial_{t}^{j} v(s)\right\|_{L^{2}} d s .
$$


Remark that in order to get (3.37) we used the fact that $\partial_{t, x} \partial_{t}^{n} v$ is infinitely small at time zero, which follows from (3.33).

The expression (3.37) is not ready for an application of Gronwall's inequality, since the integrand involves derivatives of order 2 in $x$ which are not present in the left hand side.

Lemma 3.9. Suppose that $P^{\varepsilon} v^{\varepsilon}=\mathcal{O}\left(\varepsilon^{\infty}\right)$ and the Cauchy data of $v^{\varepsilon}$ are $\mathcal{O}\left(\varepsilon^{\infty}\right)$ as in Proposition 3.8. Assume in addition that, for all $k$, there is a constant $C=C(k)$ so that

$$
\sup _{\substack{0 \leq t \leq T / \varepsilon \\|\alpha| \leq n}}\left\|\partial_{t, x}^{\alpha} v^{\varepsilon}(t)\right\|_{L^{2}\left(\mathbb{R}^{N}\right)} \leq C \varepsilon^{k}
$$

Then there is a constant $C$ independent of $\varepsilon, v^{\varepsilon}$ so that, for any $0 \leq t \leq T / \varepsilon$, the derivatives of order $n+1$ satisfy

$$
\sup _{|\alpha| \leq n+1}\left\|\partial_{t, x}^{\alpha} v^{\varepsilon}(t)\right\|_{L^{2}\left(\mathbb{R}^{N}\right)} \leq \mathcal{O}\left(\varepsilon^{\infty}\right)+C \sum_{\substack{j+|\beta|=n+1 \\|\beta| \leq 1}}\left\|\partial_{x}^{\beta} \partial_{t}^{j} v^{\varepsilon}(t)\right\|_{L^{2}\left(\mathbb{R}^{N}\right)}
$$

Proof. For $n+1 \geq|\gamma| \geq 2$ we must estimate $\partial_{x}^{\gamma} \partial_{t}^{n+1-|\gamma|} v^{\varepsilon}$. Write $\gamma=\gamma^{\prime}+\zeta$ with $|\zeta|=2$. The coercivity estimate (3.31) for $s=2$ and $\phi=\partial_{x}^{\gamma^{\prime}} \partial_{t}^{n+1-|\gamma|} v^{\varepsilon}$ shows that

$$
\left\|\partial_{x}^{\gamma} \partial_{t}^{n+1-|\gamma|} v^{\varepsilon}\right\|_{L^{2}\left(\mathbb{R}^{N}\right)} \leq C\left(\left\|G^{\varepsilon} \partial_{x}^{\gamma^{\prime}} \partial_{t}^{n+1-|\gamma|} v^{\varepsilon}\right\|_{L^{2}\left(\mathbb{R}^{N}\right)}+\frac{1}{\varepsilon^{m}}\left\|\partial_{x}^{\gamma^{\prime}} \partial_{t}^{n+1-|\gamma|} v^{\varepsilon}\right\|_{H^{1}\left(\mathbb{R}^{N}\right)}\right) .
$$

The second term in the right-hand side of (3.39) involves derivatives of order at most $n$ so, by hypothesis $(3.38)$, is $\mathcal{O}\left(\varepsilon^{\infty}\right)$. Commutation yields

$$
\left\|G^{\varepsilon} \partial_{x}^{\gamma^{\prime}} \partial_{t}^{n+1-|\gamma|} v^{\varepsilon}\right\|_{L^{2}\left(\mathbb{R}^{N}\right)} \leq\left\|\partial_{x}^{\gamma^{\prime}} \partial_{t}^{n+1-|\gamma|} G^{\varepsilon} v^{\varepsilon}\right\|_{L^{2}\left(\mathbb{R}^{N}\right)}+\left\|\left[G^{\varepsilon}, \partial_{x}^{\gamma^{\prime}} \partial_{t}^{n+1-|\gamma|}\right] v^{\varepsilon}\right\|_{L^{2}\left(\mathbb{R}^{N}\right)} .
$$

The commutator is a differential operator of degree $n$ with coefficients no larger than $\mathcal{O}\left(\varepsilon^{-\left|\gamma^{\prime}\right|}\right)$. By the inductive hypothesis the norm of the commutator is $\mathcal{O}\left(\varepsilon^{\infty}\right)$.

Write $G^{\varepsilon}=-P^{\varepsilon}+\rho^{\varepsilon} \partial_{t}^{2}$ so

$$
\partial_{x}^{\gamma^{\prime}} \partial_{t}^{n+1-|\gamma|} G^{\varepsilon} v^{\varepsilon}=-\partial_{x}^{\gamma^{\prime}} \partial_{t}^{n+1-|\gamma|} P^{\varepsilon} v^{\varepsilon}+\partial_{x}^{\gamma^{\prime}} \partial_{t}^{n+1-|\gamma|} \rho^{\varepsilon} \partial_{t}^{2} v^{\varepsilon} .
$$

The first term on the right is $\mathcal{O}\left(\varepsilon^{\infty}\right)$ by hypothesis. Expanding the second term there is one term with a derivative of order $1+n$ and the others involve derivatives of $\rho^{\varepsilon}$ times derivatives of $v^{\varepsilon}$ of order $\leq n$. Since the derivatives of $\rho^{\varepsilon}$ grow at most polynomially in $1 / \varepsilon$ these lower order derivative terms are $\mathcal{O}\left(\varepsilon^{\infty}\right)$ by the inductive hypothesis. Therefore

$$
\left\|G^{\varepsilon} \partial_{x}^{\gamma^{\prime}} \partial_{t}^{n+1-|\gamma|} v^{\varepsilon}\right\|_{L^{2}\left(\mathbb{R}^{N}\right)} \leq C\left\|\partial_{x}^{\gamma^{\prime}} \partial_{t}^{n+1-|\gamma|} \partial_{t}^{2} v^{\varepsilon}\right\|_{L^{2}\left(\mathbb{R}^{N}\right)}+\mathcal{O}\left(\varepsilon^{\infty}\right) .
$$

Injecting this in (3.39) yields

$$
\left\|\partial_{x}^{\gamma} \partial_{t}^{n+1-|\gamma|} v^{\varepsilon}\right\|_{L^{2}\left(\mathbb{R}^{N}\right)} \leq C\left\|\partial_{x}^{\gamma^{\prime}} \partial_{t}^{n+1-|\gamma|} v^{\varepsilon}\right\|_{L^{2}\left(\mathbb{R}^{N}\right)}+\mathcal{O}\left(\varepsilon^{\infty}\right)
$$

The order of the $x$ derivative on the right is lower by 2. A finite number of applications of this reduction, proves the Lemma. 
Lemma 3.9 shows that the left hand side of (3.37) dominates all derivatives of order $n+1$ so one has

$$
\sum_{|\alpha| \leq n+1}\left\|\partial_{t, x}^{\alpha} v(s)\right\|_{L^{2}} \leq \mathcal{O}\left(\varepsilon^{\infty}\right)+\int_{0}^{t} C \varepsilon \sum_{\substack{j+|\beta|=n+1 \\|\beta| \leq 2}}\left\|\partial_{x}^{\beta} \partial_{t}^{j} v(s)\right\|_{L^{2}} d s .
$$

The sum in the integrand is smaller than the sum on the left hand side. Gronwall's Lemma completes the proof of the inductive step.

Theorem 3.10. Let $v^{\varepsilon}(t, x)$ be the approximate solution defined in Theorem 3.5 and $u^{\varepsilon}(t, x)$ be the unique solution of the initial value problem

$$
P^{\varepsilon}\left(t, x, \partial_{t, x}\right) u^{\varepsilon}=0,\left.\quad \partial_{t}^{k} u^{\varepsilon}\right|_{t=0}=\left.\partial_{t}^{k} v^{\varepsilon}\right|_{t=0}, \quad \text { for } \quad k=0,1 .
$$

Then for any $T>0, \alpha \in \mathbb{N}^{1+N}$, and $n \in \mathbb{N}$, there is a constant $C>0$ so that

$$
\sup _{|t| \leq T}\left\|\partial_{t, x}^{\alpha}\left(u^{\varepsilon}-v^{\varepsilon}\right)\right\|_{L^{2}\left(\mathbb{R}^{N}\right)} \leq C \varepsilon^{n}
$$

Proof. This estimate follows from Proposition 3.8 since the error

$$
E^{\varepsilon}:=u^{\varepsilon}(t, x)-e^{2 \pi i S / \varepsilon} W(\varepsilon, t, x, x / \varepsilon)
$$

satisfies

$$
\forall n, \forall s, \quad\left\|P^{\varepsilon}\left(t, x, \partial_{t, x}\right) E^{\varepsilon}\right\|_{H^{s}\left([0, T] \times \mathbb{R}^{N}\right)}=\mathcal{O}\left(\varepsilon^{n}\right),
$$

and, the Cauchy data of $E^{\varepsilon}$ vanish identically.

Remark 3.11. If one is interested in an $\mathcal{O}\left(\varepsilon^{n}\right)$ error estimate in (3.40) for a fixed integer $n$, it suffices to truncate the approximate solution $v^{\varepsilon}$, defined in (3.3), at order $n+|\alpha|$.

\section{Diffractive GeOmetric optics For Bloch WAVE PACKets}

4.1. The long time ansatz. This section is devoted to long times $t$ of order $1 / \varepsilon$. As in the case of equations with constant coefficients and linear phases [14], this time scale leads to envelope equations of Schrödinger type. The support of the leading term in the asymptotic solution extends beyond the tube of rays with feet in the initial data. For that reason it is called diffractive.

In order for the modulations to affect the leading order asymptotics at times of order $1 / \varepsilon$ and not before consider perturbations smaller than in the preceding section.

Hypotheses. The coefficients are given by

$$
\rho^{\varepsilon}=\rho_{0}(x / \varepsilon)+\varepsilon^{2} \rho_{1}(t, x, x / \varepsilon), \quad A^{\varepsilon}=A_{0}(x / \varepsilon)+\varepsilon^{2} A_{1}(t, x, x / \varepsilon),
$$

with $\rho_{1}, A_{1}$ satisfying (1.3), (1.4). In addition the important invariance hypothesis (1.12) is satisfied, as well as assumption (2.2) that $\lambda_{n}\left(\theta_{0}\right) \neq 0$ is a simple eigenvalue. 
These modulations are weaker by one power of $\varepsilon$ than in the preceding section. If these weaker perturbations were considered for times $t=\mathcal{O}(1)$, they would not influence the leading order term in the asymptotic expansion. We consider again a solution $u^{\varepsilon}$ of (1.1). Motivated by the constant coefficient case and the case of purely periodic media in Section 2.4 , consider linear phases,

$$
S(t, x)=\omega t+\theta \cdot x
$$

and a three scale ansatz of WKB type,

$$
u^{\varepsilon}(t, x) \sim e^{2 \pi i S(t, x) / \varepsilon}\left(w_{0}\left(\varepsilon t, t, x, \frac{x}{\varepsilon}\right)+\varepsilon w_{1}\left(\varepsilon t, t, x, \frac{x}{\varepsilon}\right)+\cdots\right),
$$

where the $w_{j}(\mathcal{T}, t, x, y)$ are periodic functions of $y$ with period one. The key feature is the slow time scale $\mathcal{T}=\varepsilon t$ which becomes relevant for $t$ of order $1 / \varepsilon$. The problem addressed here is to take modulations as in (4.1) and ask how the solutions constructed in the preceding section behave on the longer time scale. At those times modulations satisfying (4.1) can affect the leading term in the expansions.

For $t \sim 1 / \varepsilon$ one expects solutions to reach $x \sim 1 / \varepsilon$. The ordering of the terms in (4.2) is measured by their rate of decay as $\varepsilon \rightarrow 0$. In order for that ordering to be respected for times $t \sim 1 / \varepsilon$ we require that the $w_{j}$ grow sublinearly in $(t, x)$. For example, if $w_{1}$ grew linearly in $t, x$, then for times $t \sim 1 / \varepsilon$ the term $\varepsilon w_{1}$ would be $\mathcal{O}(1)$ so might not have size smaller than the leading term. To avoid this we suppose that for all $j, T$,

$$
\lim _{|t, x| \rightarrow \infty} \sup _{0 \leq \mathcal{T} \leq T, y \in \mathbb{T}^{N}} \frac{\left|w_{j}(\mathcal{T}, t, x, y)\right|}{|t, x|}=0 .
$$

The profiles that we construct will satisfy the stronger condition that the $w_{j}$ are bounded. This phenomenon is already present in the constant coefficient case [14].

Given $w_{j}$, Borel's Theorem allows us to choose smooth $W(\varepsilon, \mathcal{T}, t, x, y)$, periodic in $y$ with Taylor expansion in $\varepsilon$,

$$
W(\varepsilon, \mathcal{T}, t, x, y) \sim w_{0}(\mathcal{T}, t, x, y)+\varepsilon w_{1}(\mathcal{T}, t, x, y)+\cdots
$$

Approximate solutions are defined by

$$
v^{\varepsilon}(t, x):=e^{2 \pi i S / \varepsilon} W(\varepsilon, \varepsilon t, t, x, x / \varepsilon)
$$

Then

$$
e^{-2 \pi i S / \varepsilon} P^{\varepsilon} v^{\varepsilon}=e^{-2 \pi i S / \varepsilon} P^{\varepsilon}\left(e^{2 \pi i S / \varepsilon} W(\varepsilon, \varepsilon t, t, x, x / \varepsilon)\right)=R(\varepsilon, \varepsilon t, t, x, x / \varepsilon),
$$


with

$$
\begin{aligned}
R(\varepsilon, \mathcal{T}, t, x, y) & =e^{-2 \pi i S / \varepsilon}\left[\rho^{\varepsilon}\left(\partial_{t}+\varepsilon \partial_{\mathcal{T}}\right)^{2}\right. \\
& \left.-\left(\operatorname{div}_{x}+\frac{\operatorname{div}_{y}}{\varepsilon}\right) A^{\varepsilon}\left(\operatorname{grad}_{x}+\frac{\operatorname{grad}_{y}}{\varepsilon}\right)\right]\left(e^{2 \pi i S / \varepsilon} W(\varepsilon, \mathcal{T}, t, x, y)\right) \\
& =\left[\left(\rho_{0}+\varepsilon^{2} \rho_{1}\right)\left(\frac{2 \pi i \omega}{\varepsilon}+\partial_{t}+\varepsilon \partial_{\mathcal{T}}\right)^{2}\right. \\
& \left.-\left(\frac{\operatorname{div}_{y}+2 \pi i \theta}{\varepsilon}+\operatorname{div}_{x}\right)\left(A_{0}+\varepsilon^{2} A_{1}\right)\left(\frac{\operatorname{grad}_{y}+2 \pi i \theta}{\varepsilon}+\operatorname{grad}_{x}\right)\right] W .
\end{aligned}
$$

Equation (4.5) implies that there are uniquely determined $r_{j}$ so that

$$
R(\varepsilon, \mathcal{T}, t, x, y) \sim \sum_{j=-2}^{\infty} \varepsilon^{j} r_{j}(\mathcal{T}, t, x, y) .
$$

Compared to the preceding section there are two differences. The perturbation of the coefficients is $\mathcal{O}\left(\varepsilon^{2}\right)$ rather than $\mathcal{O}(\varepsilon)$, and there is the $\varepsilon \partial_{\mathcal{T}}$ term.

4.2. The leading profile. We expand the operator on the right in (4.5) in powers of $\varepsilon$, and keep the leading three orders

$$
\varepsilon^{-2} \mathbb{L}+\varepsilon^{-1} \mathbb{M}+\varepsilon^{0} \mathbb{N} .
$$

The term $\mathbb{L}$ is as in (3.5),

$$
\mathbb{M}\left(y, \partial_{t}, \partial_{x}, \partial_{y}\right):=4 \pi i \rho_{0} \omega \partial_{t}-\left(2 \pi i \theta+\operatorname{div}_{y}\right) A_{0} \operatorname{grad}_{x}-\operatorname{div}_{x} A_{0}\left(2 \pi i \theta+\operatorname{grad}_{y}\right),
$$

and,

$$
\begin{array}{r}
\mathbb{N}\left(t, x, y, \partial_{\mathcal{T}}, \partial_{t}, \partial_{x}, \partial_{y}\right):=4 \pi i \rho_{0} \omega \partial_{\mathcal{T}}+\left(\rho_{0} \frac{\partial^{2}}{\partial t^{2}}-\operatorname{div}_{x} A_{0} \operatorname{grad}_{x}\right)+ \\
\left(\rho_{1}(2 \pi i \omega)^{2}-\left(\operatorname{div}_{y}+2 \pi i \theta\right) A_{1}\left(\operatorname{grad}_{y}+2 \pi i \theta\right)\right) .
\end{array}
$$

The operator $\mathbb{M}$ is simpler than in the preceding section. Here it involves only $\rho_{0}$ and $A_{0}$. The modulations $\rho_{1}, A_{1}$ appear in $\mathbb{N}$. At the symbol level one has,

(4.8) $\mathbb{M}\left(y, \partial_{t}, h, \partial_{y}\right):=4 \pi i \rho_{0}(y) \omega \partial_{t}-\left(\operatorname{div}_{y}+2 \pi i \theta\right) A_{0}(y) h-h A_{0}(y)\left(\operatorname{grad}_{y}+2 \pi i \theta\right)$,

where $h$ replaces $\partial_{x}$. The leading terms in the residual are,

$$
r_{-2}=\mathbb{L} w_{0}, \quad r_{-1}=\mathbb{L} w_{1}+\mathbb{M} w_{0}, \quad, \quad r_{0}=\mathbb{L} w_{2}+\mathbb{M} w_{1}+\mathbb{N} w_{0} .
$$

The relation $r_{-2}=0$ leads to (3.8), the dispersion relation (3.10), the Definition 3.1 of $\mathbb{K}$, $\Pi, Q$, and (3.11), as in the preceding section. Fix $\omega, \theta_{0}, \mathcal{V}$ as before.

Since $r_{-2}$ is in the image of $\mathbb{L}$, one automatically has $\Pi r_{-2}=0$. The equation $r_{-2}=0$ is equivalent to $Q r_{-2}=0$. For $j \geq-1$, each equation $r_{j}=0$ is split into two equations, $\Pi r_{j}=0$ and $Q r_{j}=0$. 
The equation $r_{-1}=0$ is,

$$
\mathbb{L} w_{1}+\mathbb{M} w_{0}=0 .
$$

Since $w_{0}=\Pi w_{0}$ and $\Pi \mathbb{L}=0$, the equation $\Pi r_{-1}=0$ is equivalent to

$$
\Pi \mathbb{M} \Pi w_{0}=0 .
$$

With the simpler form of $\mathbb{M}$ in (4.6) (without the perturbations $\rho_{1}, A_{1}$ ), Proposition 3.2 shows that,

$$
\Pi \mathbb{M} \Pi w=4 \pi i\left(\partial_{t}+\mathcal{V} . \partial_{x}\right) \Pi w, \quad \mathcal{V}:=-\nabla_{\theta} \omega\left(\theta_{0}\right)
$$

SO

$$
\left(\partial_{t}+\mathcal{V} . \partial_{x}\right) w_{0}=0
$$

Thus there is a reduced $\mathbb{K}$ valued profile $\widetilde{w}_{0}(\mathcal{T}, x)$ so that

$$
w_{0}(\mathcal{T}, t, x)=\widetilde{w}_{0}(\mathcal{T}, x-\mathcal{V} t) .
$$

It remains to determine the $\mathbb{K}$ valued function $\widetilde{w}_{0}(\mathcal{T}, x)$ of $1+N$ variables. One needs a dynamic equation in $\mathcal{T}$. The reader is reminded that $\widetilde{w}_{0}$ is $\mathbb{K}$ valued and $\mathbb{K}$ consists of functions of $y$, so $\widetilde{w}_{0}$ is actually a function of $(t, x, y)$.

The equation $Q r_{-1}=0$ yields

$$
(I-\Pi) w_{1}=-Q \mathbb{M} w_{0} .
$$

Equation (4.11) together with the fact that the coefficients of $\mathbb{M}$ depend only on $y$ imply that the right hand side is a function of $(\mathcal{T}, x-\mathcal{V} t, y)$. The same is therefore true of the left hand side, so

$$
\left(\partial_{t}+\mathcal{V} . \partial_{x}\right)\left((I-\Pi) w_{1}\right)=0
$$

This exhausts the information from $r_{-2}, r_{-1}$. The equations (4.13) and (4.14) are important steps toward determining the first corrector $w_{1}$, and are also needed to derive the equations determining the leading profile $w_{0}$.

The equation $\Pi r_{0}=0$ yields the Schrödinger equation determining the dynamics of $\widetilde{w}_{0}$. The fact that the leading profile $w_{0}$ is determined from three orders in the residual is a reflection of the three scale structure of the asymptotics.

Multiply $r_{0}$ by $\Pi$ and decompose $w_{0}, w_{1}$ along $\mathbb{K} \oplus \mathbb{K}^{\perp}$ using (3.11) and (4.13) to find

$$
\Pi \mathbb{M}\left(\Pi w_{1}-Q \mathbb{M} w_{0}\right)+\Pi \mathbb{N} \Pi w_{0}=0
$$

This yields two equations. Multiply by $\partial_{t}+\mathcal{V} . \partial_{x}$ and use (4.10), (4.11), and most importantly the invariance of the coefficients, (1.12), to eliminate the $w_{0}$ terms leaving,

$$
\left(\partial_{t}+\mathcal{V} . \partial_{x}\right)^{2}\left(\Pi w_{1}\right)=0 .
$$


This shows that the restriction of $\Pi w_{1}$ to each ray, $t \mapsto(t, x+\mathcal{V} t)$, is a linear function of $t$. Since by assumption (4.3) each profile is required to have sublinear growth, the linear function must be constant, so,

$$
\left(\partial_{t}+\mathcal{V} . \partial_{x}\right)\left(\Pi w_{1}\right)=0 .
$$

Thus, the single equation (4.15) implies two equations, (4.16), and

$$
\Pi \mathbb{N} \Pi w_{0}-\Pi \mathbb{M} Q \mathbb{M} \Pi w_{0}=0 .
$$

Combining (4.16) and (4.14) yields

$$
\left(\partial_{t}+\mathcal{V} . \partial_{x}\right) w_{1}=0
$$

Thus, there is a reduced profile $\widetilde{w}_{1}(\mathcal{T}, x, y)$ so that $w_{1}(\mathcal{T}, t, x, y)=\widetilde{w}_{1}(\mathcal{T}, x-\mathcal{V} t, y)$. This corrector is not in general $\mathbb{K}$ valued. $(I-\Pi) \widetilde{w}_{1}$ is determined in (4.13). The remaining part $\Pi \widetilde{w}_{1}$ will be determined after we find $\widetilde{w}_{0}$.

Proposition 4.1. On smooth functions $w(\mathcal{T}, t, x, y)$ which satisfy $\left(\partial_{t}+\mathcal{V} . \partial_{x}\right) w=0$,

$$
(\Pi \mathbb{N} \Pi-\Pi \mathbb{M} Q \mathbb{M} \Pi) w=\left(4 \pi i \omega \partial_{\mathcal{T}}-\omega \nabla_{\theta}^{2} \omega\left(\partial_{x}, \partial_{x}\right)+\gamma(t, x)\right) \Pi w,
$$

where $\gamma$ is defined in (3.17).

Proof. The definitions of $\Pi$ in Subsection 3.2, of $\mathbb{N}$ in (4.7), and the normalization (1.8) imply that the $\partial_{\mathcal{T}}$ term on the left in (4.19) is equal to

$$
\Pi 4 \pi i \omega \rho_{0} \partial_{\mathcal{T}} \Pi w=4 \pi i \omega \partial_{\mathcal{T}}(\Pi w) . .
$$

Similarly the zero order term (with respect to $t$ and $x$ ) from $\Pi \mathbb{N} \Pi w$ is equal to $\gamma(t, x) w$. Use $\partial_{t} w=-\mathcal{V} . \partial_{x} w$ to conclude that

$$
\begin{aligned}
& (\Pi \mathbb{N} \Pi-\Pi \mathbb{M} Q \mathbb{M} \Pi) w= \\
& \quad\left(4 \pi i \omega \partial_{\mathcal{T}}+\Pi\left(\rho_{0}\left(\mathcal{V} . \partial_{x}\right)^{2}-\operatorname{div}_{x} A_{0} \operatorname{grad}_{x}-\mathbb{M} Q \mathbb{M}\right) \Pi+\gamma(t, x)\right) \Pi w .
\end{aligned}
$$

The second order terms in $x$ come from the $\left(\mathcal{V} . \partial_{x}\right)^{2}$, the two factors of $\mathbb{M}$, each of which is first order in $\partial_{t, x}$, and, the $\operatorname{div}_{x} A_{0} \operatorname{grad}_{x}$ term. They simplify thanks to the identities of Proposition 3.3 as we now explain.

With $h=\left(h_{1}, h_{2}, \ldots, h_{N}\right) \in \mathbb{R}^{N}$ fixed, apply (3.22) for $\theta:=h \alpha, \alpha \in \mathbb{R}$. Then the derivative with respect to $\alpha$ is ${ }^{\prime}=h . \partial_{\theta}=\sum h_{j} \partial / \partial \theta_{j}$, and,

$$
\mathbb{L}^{\prime}=-4 \pi^{2} \rho_{0}\left(h . \partial_{\theta}\right)\left(\omega^{2}\right)-2 \pi i h A_{0}\left(\operatorname{grad}_{y}+2 \pi i \theta\right)-\left(\operatorname{div}_{y}+2 \pi i \theta\right) A_{0} 2 \pi i h .
$$

From (4.8) one has

$-2 \pi i h A_{0}\left(\operatorname{grad}_{y}+2 \pi i \theta\right)-\left(\operatorname{div}_{y}+2 \pi i \theta\right) A_{0} 2 \pi i h=2 \pi i \mathbb{M}\left(y, \partial_{t}, h, \partial_{y}\right)+2 \pi i\left(-4 \pi i \omega \rho_{0} \partial_{t}\right)$.

Use this in (4.21) to find

$$
\mathbb{L}^{\prime}=-4 \pi^{2} \rho_{0}\left(h . \partial_{\theta}\right)\left(\omega^{2}\right)+2 \pi i \mathbb{M}\left(y, \partial_{t}, h, \partial_{y}\right)+8 \pi^{2} \omega \rho_{0} \partial_{t} .
$$


Differentiate (4.21) to find,

$$
\mathbb{L}^{\prime \prime}=-4 \pi^{2} \rho_{0}\left(h . \partial_{\theta}\right)^{2}\left(\omega^{2}\right)-8 \pi i h A_{0} \pi i h=-4 \pi^{2} \rho_{0} \nabla_{\theta}^{2}\left(\omega^{2}\right)(h, h)+8 \pi^{2} h A_{0} h,
$$

where,

$$
\nabla_{\theta}^{2}\left(\omega^{2}\right)(h, k):=\sum_{i=1}^{N} \sum_{j=1}^{N} \frac{\partial^{2}\left(\omega^{2}\right)}{\partial \theta_{i} \partial \theta_{j}} h_{i} k_{j}
$$

Plug (4.22) into (3.23). Since $\Pi Q=Q \Pi=0$, each of the terms involving

$$
-4 \pi^{2} \rho_{0}\left(h . \partial_{\theta}\right)\left(\omega^{2}\right)+8 \pi^{2} \omega \rho_{0} \partial_{t}
$$

vanishes. Therefore,

$$
\Pi \mathbb{L}^{\prime} Q \mathbb{L}^{\prime} \Pi=-4 \pi^{2} \Pi \mathbb{M}\left(y, \partial_{t}, h, \partial_{y}\right) Q \mathbb{M}\left(y, \partial_{t}, h, \partial_{y}\right) \Pi .
$$

Using this and (4.23) in (3.23) yields,

$\Pi\left(8 \pi^{2} h A_{0} h-4 \pi^{2} \rho_{0} \nabla_{\theta}^{2}\left(\omega^{2}\right)(h, h)\right) \Pi+8 \pi^{2} \Pi \mathbb{M}\left(y, \partial_{t}, h, \partial_{y}\right) Q \mathbb{M}\left(y, \partial_{t}, h, \partial_{y}\right) \Pi=0$.

Polarization implies equality of the associated symmetric bilinear forms,

$\Pi\left(8 \pi^{2} h A_{0} k-4 \pi^{2} \rho_{0} \nabla_{\theta}^{2}\left(\omega^{2}\right)(h, k)\right) \Pi+8 \pi^{2} \Pi \mathbb{M}\left(y, \partial_{t}, h, \partial_{y}\right) Q \mathbb{M}\left(y, \partial_{t}, k, \partial_{y}\right) \Pi=0$.

Dividing by $8 \pi^{2}$ and replacing $h$ and $k$ by $\partial_{x}$ yields

$$
\Pi \mathbb{M}\left(y, \partial_{t}, \partial_{x}, \partial_{y}\right) Q \mathbb{M}\left(y, \partial_{t}, \partial_{x}, \partial_{y}\right) \Pi=\Pi\left(\frac{\rho_{0}}{2} \nabla_{\theta}^{2}\left(\omega^{2}\right)\left(\partial_{x}, \partial_{x}\right)-\operatorname{div}_{x} A_{0}(y) \operatorname{grad}_{x}\right) \Pi
$$

Use this in (4.20) to find that ( $\mathbb{N} \Pi-\Pi \mathbb{M} Q \mathbb{M} \Pi) w$ is equal to

$$
\left(4 \pi i \omega \partial_{\mathcal{T}}+\Pi\left(\rho_{0}\left(\mathcal{V} . \partial_{x}\right)^{2}-\frac{\rho_{0}}{2} \nabla_{\theta}^{2}\left(\omega^{2}\right)\left(\partial_{x}, \partial_{x}\right)\right) \Pi+\gamma(t, x)\right) \Pi w .
$$

Taking account of the $\mathbb{K}$ valued character of $\Pi w$, the definition of $\Pi$ and the normalization (1.8), this is equal to

$$
\left(4 \pi i \omega \partial_{\mathcal{T}}+\left(\mathcal{V} . \partial_{x}\right)^{2}-\frac{1}{2} \nabla_{\theta}^{2}\left(\omega^{2}\right)\left(\partial_{x}, \partial_{x}\right)+\gamma(t, x)\right) \Pi w .
$$

Using,

$$
\nabla_{\theta}^{2}\left(\omega^{2}\right)\left(\partial_{x}, \partial_{x}\right)=2 \omega \nabla_{\theta}^{2} \omega\left(\partial_{x}, \partial_{x}\right)+2\left(\nabla_{\theta} \omega . \partial_{x}\right)^{2}=2 \omega \nabla_{\theta}^{2} \omega\left(\partial_{x}, \partial_{x}\right)+2\left(\mathcal{V} . \partial_{x}\right)^{2}
$$

yields,

$$
\left(4 \pi i \omega \partial_{\mathcal{T}}-\omega \nabla_{\theta}^{2} \omega\left(\partial_{x}, \partial_{x}\right)+\gamma(t, x)\right) \Pi w
$$

This proves Proposition 4.1. 
The leading profile must satisfy (4.11) and combining (4.11), (4.17), and (4.19) yields the Schrödinger equation

$$
\left(4 \pi i \omega \partial_{\mathcal{T}}-\omega \nabla_{\theta}^{2} \omega\left(\partial_{x}, \partial_{x}\right)+\gamma(t, x)\right) w_{0}=0 .
$$

Applying the operator $\partial_{t}+\mathcal{V} . \partial_{x}$ to $(4.24)$ and using $(4.11)$ yields $\left(\left(\partial_{t}+\mathcal{V} . \partial_{x}\right) \gamma\right) w_{0}=0$ so that solvability requires that $\left(\partial_{t}+\mathcal{V} . \partial_{x}\right) \gamma=0$ on the support of $w_{0}$.

Conversely, when $\gamma$ satisfies (1.12), introducing $\widetilde{\gamma}$ such that $\gamma(t, x)=\widetilde{\gamma}(x-\mathcal{V} t)$, equation (4.24) is equivalent to

$$
\Pi w_{0}=w_{0}=\widetilde{w}_{0}(\mathcal{T}, x-\mathcal{V} t), \quad\left(4 \pi i \omega \partial_{\mathcal{T}}-\omega \nabla_{\theta}^{2} \omega\left(\partial_{x}, \partial_{x}\right)+\widetilde{\gamma}(x)\right) \widetilde{w}_{0}=0 .
$$

The leading profile $\widetilde{w}_{0}(\mathcal{T}, x)$ is uniquely determined from its initial data as a tempered solution of (4.25)

Example 4.2. In the case of purely periodic coefficients one recovers the Schrödinger equation,

$$
4 \pi i \partial_{\mathcal{T}} \widetilde{w}_{0}-\nabla_{\theta}^{2} \omega\left(\partial_{x}, \partial_{x}\right) \widetilde{w}_{0}=0
$$

which agrees with (2.11).

Example 4.3. Even more special is the case of the speed one constant coefficient wave equation on $\mathbb{R}^{N}$ where (4.26) is the standard Schrödinger approximation with $\nabla_{\theta}^{2} \omega$ equal to \pm the partial laplacian orthogonal to the direction $\theta_{0}$.

The results of this section are summarized by the following proposition.

Proposition 4.4. The leading profile is a $\mathbb{K}$ valued function $\widetilde{w}_{0}(\mathcal{T}, x-\mathcal{V} t)$ where $\widetilde{w}_{0}$ is determined from its initial data at $\mathcal{T}=0$ as the unique tempered solution of (4.25). The first corrector $w_{1}$ satisfies (4.18) and its projection $w_{1}$ orthogonal to $\mathbb{K}$ is given by (4.13). These prescriptions are equivalent to the equations $r_{-2}=r_{-1}=\Pi r_{0}=0$.

4.3. Determination of the correctors. The hard work for problems satisfying (1.12) is over. We show how the computation continues by determining the first corrector $w_{1}$. The projection $(I-\Pi) w_{1}$ is already determined. We show that $\Pi w_{1}$ and $Q w_{2}$ are determined from the pair of equations $Q r_{0}=0$ and $\Pi r_{1}=0$. Inductively, $\Pi w_{J}$ and $Q w_{J+1}$ are determined in the same way from the pair of equations $Q r_{J-1}=0$ and $\Pi r_{J}=0$ and the values of the $w_{0}, \ldots, w_{J-1},(I-\Pi) w_{J}$.

Using (4.9), the equation $Q r_{0}=0$ holds if and only if

$$
(I-\Pi) w_{2}=-Q \mathbb{M} w_{1}-Q \mathbb{N} w_{0},
$$

In particular using (1.12) and the invariance of $w_{0}, w_{1}$ along rays yields

$$
\left(\partial_{t}+\mathcal{V} . \partial_{x}\right)\left((I-\Pi) w_{2}\right)=0 \text {. }
$$


The residual $r_{1}$ is given by

$$
r_{1}=\mathbb{L} w_{3}+\mathbb{M} w_{2}+\mathbb{N} w_{1}+F_{1}\left(w_{0}\right),
$$

where the term $F_{1}$ is determined entirely from $w_{0}$ and its partial derivatives. Multiply by $\Pi$ and decompose $w_{1}, w_{2}$ along $\mathbb{K} \oplus \mathbb{K}^{\perp}$ to find, using (4.13) and $Q r_{0}=0$,

(4.29) $\Pi \mathbb{M}\left(\Pi w_{2}-Q \mathbb{M}\left(\Pi w_{1}-Q \mathbb{M} w_{0}\right)-Q \mathbb{N} w_{0}\right)+\Pi \mathbb{N}\left(\Pi w_{1}-Q \mathbb{M} w_{0}\right)=-\Pi F_{1}\left(w_{0}\right)$.

Multiply by $\partial_{t}+\mathcal{V} . \partial_{x}$ to find using the hypothesis (1.12),

$$
\left(\partial_{t}+\mathcal{V} . \partial_{x}\right)^{2} \Pi w_{2}=0 .
$$

By assumption (4.3) (sublinearity along rays) it follows that

$$
\left(\partial_{t}+\mathcal{V} . \partial_{x}\right) \Pi w_{2}=0
$$

Combined with (4.28) this implies that $\left(\partial_{t}+\mathcal{V} . \partial_{x}\right) w_{2}=0$. Thus, in view of $(4.10)$, the $\Pi \mathbb{M} w_{2}$ term vanishes and there is a reduced profile, $\widetilde{w}_{2}(\mathcal{T}, x, y)$, so that

$$
w_{2}(\mathcal{T}, t, x, y)=\widetilde{w}_{2}(\mathcal{T}, x-\mathcal{V} t, y)
$$

Then, (4.29) becomes

$$
(\Pi \mathbb{N} \Pi-\Pi \mathbb{M} Q \mathbb{M} \Pi) w_{1}=-\Pi F_{2}\left(w_{0}\right),
$$

with $F_{2}$ determined from $w_{0}$ and its derivatives. When $\gamma$ satisfies (1.12), Proposition 4.1 shows that (4.30) holds if and only if

$$
\left(4 \pi i \omega \partial_{\mathcal{T}}-\omega \nabla_{\theta}^{2} \omega\left(\partial_{x}, \partial_{x}\right)+\widetilde{\gamma}(x)\right)\left(\Pi \widetilde{w}_{1}\right)=-\Pi F_{2}\left(\widetilde{w}_{0}\right) .
$$

This equation along with (4.13) completely determines $w_{1}$ from the initial values $\left.\widetilde{w}_{1}\right|_{\mathcal{T}=0}$.

In addition, (4.27) determines $(I-\Pi) w_{2}$. These determinations together with the earlier ones are equivalent to the equations $r_{-2}=r_{-1}=r_{0}=\Pi r_{1}=0$. The new equations are $Q r_{0}=\Pi r_{1}=0$. This completes the second step of the inductive determination of the profiles $w_{j}$ from the initial values of $\Pi \widetilde{w}_{j}$. When they are all so determined, all the residuals $r_{j}$ vanish.

Remark 4.5. In homogenization problems one often uses $(I-\Pi) w_{1}$ as part of test functions. The common expressions are complicated involving $\theta$ derivatives of $\psi_{n}(x, \theta)$ (see for example [4]). If one writes out our formula in detail one recovers those formulas. The present formulation is well adapted to a systematic inductive argument.

These computations yield the first of the following Theorems. We use the Schwartz $\operatorname{class} \mathcal{S}\left(\mathbb{R}_{x}^{N} \times \mathbb{T}_{y}^{N}\right)$ defined by

$$
\forall \alpha, \beta \quad \sup _{\mathbb{R}_{x}^{N} \times \mathbb{T}_{y}^{N}}\left|x^{\beta} \partial_{x, y}^{\alpha} w(x, y)\right|<\infty
$$


This class is chosen as it gives the most structured of solutions. A result with the milder class $\cap_{s} H^{s}\left(\mathbb{R}^{N} \times \mathbb{T}^{N}\right)$ is stated in the introduction.

Theorem 4.6. Suppose that $\theta_{0}$ and $\omega$ satisfy the dispersion relation (3.10) at a simple eigenvalue $\lambda_{n}\left(\theta_{0}\right) \neq 0$. Given Schwartz class $\mathbb{K}$ valued functions $g_{j}(x, y)$, there are uniquely determined $w_{j}(\mathcal{T}, t, x, y)=\widetilde{w}_{j}(\mathcal{T}, x-\mathcal{V} t, y)$ with $\widetilde{w}_{j} \in C^{\infty}\left(\left[0, \infty\left[\mathcal{T} ; \mathcal{S}\left(\mathbb{R}_{x}^{N} \times \mathbb{T}_{y}^{N}\right)\right)\right.\right.$ so that

$$
\left.\widetilde{w}_{0}\right|_{t=0}=g_{0}, \quad \forall j \geq\left. 1 \quad \Pi \widetilde{w}_{j}\right|_{t=0}=g_{j}, \quad \text { and } \quad \forall j, t, x, y, \quad r_{j}(t, x, y)=0 .
$$

The leading term $\widetilde{w}_{0}$ is $\mathbb{K}$ valued and is determined from the Schrödinger equation (4.25).

Theorem 4.7. Suppose that $g_{j}(x, y)$ are Schwartz class $\mathbb{K}$ valued functions. Let $w_{j}$ be the profiles constructed in the preceding theorem. Choose $\widetilde{W}(\varepsilon, \mathcal{T}, x, y) \in C^{\infty}\left(\left[0,1\left[\times\left[0, \infty\left[\times \mathbb{R}^{N} \times\right.\right.\right.\right.\right.$ $\left.\mathbb{T}^{N}\right)$ with Taylor series in $\varepsilon$,

$$
\widetilde{W}(\varepsilon, \mathcal{T}, x, y) \sim \sum_{j=0}^{\infty} \varepsilon^{j} \widetilde{w}_{j}(\mathcal{T}, x, y),
$$

such that, for any $\alpha \in \mathbb{N}^{1+N}, \beta \in \mathbb{N}^{N}, m \in \mathbb{N}, T>0$, there exists a constant $C>0$ satisfying, $\forall \varepsilon>0$,

$$
\sup _{\{0 \leq \mathcal{T} \leq T\} \times \mathbb{R}^{N} \times \mathbb{T}^{N}}\left|x^{\beta} \partial_{\mathcal{T}, x}^{\alpha}\left(\widetilde{W}-\sum_{j=0}^{m} \varepsilon^{j} \widetilde{w}_{j}\right)\right| \leq C \varepsilon^{m+1} .
$$

Define

$$
v^{\varepsilon}(t, x):=e^{2 \pi i S / \varepsilon} \widetilde{W}(\varepsilon, \varepsilon t, x-\mathcal{V} t, x / \varepsilon) .
$$

Then $P^{\varepsilon} v^{\varepsilon}=\mathcal{O}\left(\varepsilon^{\infty}\right)$ in the sense that, for all $\alpha \in \mathbb{N}^{1+N}, \beta \in \mathbb{N}^{N}, m \in \mathbb{N}, T>0$, there exists a constant $C>0$ satisfying, $\forall \varepsilon>0$,

$$
\sup _{\{0 \leq t \leq T / \varepsilon\} \times \mathbb{R}^{N} \times \mathbb{T}^{N}}\left|x^{\beta} \partial_{t, x}^{\alpha}\left(P^{\varepsilon} v^{\varepsilon}\right)\right| \leq C \varepsilon^{n} .
$$

Proof. The proof is like that of Theorem 3.5.

Theorem 4.8. With notation and hypotheses of the preceding theorem, let $u^{\varepsilon}(t, x)$ be the unique solution of the initial value problem

$$
P^{\varepsilon}\left(t, x, \partial_{t, x}\right) u^{\varepsilon}=0,\left.\quad \partial_{t}^{k} u^{\varepsilon}\right|_{t=0}=\left.\partial_{t}^{k} v^{\varepsilon}\right|_{t=0}, \quad \text { for } \quad k=0,1 .
$$

Then for any $T>0, \alpha \in \mathbb{N}^{1+N}$, and, $n \in \mathbb{N}$, there is a constant $C>0$ so that

$$
\sup _{|t| \leq T / \varepsilon}\left\|\partial_{t, x}^{\alpha}\left(u^{\varepsilon}-v^{\varepsilon}\right)\right\|_{L^{2}\left(\mathbb{R}_{x}^{N}\right)} \leq C \varepsilon^{n} .
$$


Proof. As in the proof of Proposition 3.8, one first establishes that

$$
\left\|\left.\partial_{t, x}^{\alpha}\left(u^{\varepsilon}-v^{\varepsilon}\right)\right|_{t=0}\right\|_{L^{2}\left(\mathbb{R}_{x}^{N}\right)} \leq C \varepsilon^{n}
$$

Then the Theorem is an immediate consequence of the residual estimate in the preceding Theorem and the stability estimate (3.34). The latter estimate was proved for stronger perturbations and is true without modification in the present context.

\section{Modulations that are not Constant on Group lines}

This section treats modulations $\rho_{1}(t, x, y), A_{1}(t, x, y)$ that are not constant on group lines. The coefficients are given by

$$
\rho^{\varepsilon}=\rho_{0}(x / \varepsilon)+\varepsilon^{2} \rho_{1}(t, x, x / \varepsilon), \quad A^{\varepsilon}=A_{0}(x / \varepsilon)+\varepsilon^{2} A_{1}(t, x, x / \varepsilon),
$$

with $\rho_{1}, A_{1}$ satisfying (1.3), (1.4), but not the invariance hypothesis (1.12).

For (1.12) to hold for several distinct group velocities is a very strong condition. For example, if $\mathcal{V}_{\mu}$ is a family of such velocities so that $\partial_{t}+\mathcal{V}_{\mu} . \partial_{x}$ span $\mathbb{R}^{1+N}$, then the only $\rho_{1}, A_{1}$ which satisfy (1.12) for all these velocities are periodic functions of $y$ which do not depend on $(t, x)$. The conditions imposed in this section do not have this sort of defect.

5.1. Ray averages. Wave packets move with the group velocity $\mathcal{V}$. An observer moving at this speed sees the coefficients along the rays $(t, x+\mathcal{V} t)$. On such a ray, $\gamma=\gamma(t, x+\mathcal{V} t)$. In this section the hypothesis that $\gamma$ is constant on rays is replaced by a weaker hypothesis concerning the average of $\gamma$ on rays.

Begin by assuming that the averages on rays,

$$
\lim _{T \rightarrow+\infty} \frac{1}{T} \int_{0}^{T} \gamma(t, x+\mathcal{V} t) d t:=\widetilde{\gamma}(x), \quad \text { exists uniformly in } x .
$$

We need more than this. The function $\widetilde{\gamma}(x)$ is the average on the ray intersecting $t=0$ at $x$. The ray passing through the point $(t, x)$ intersects $t=0$ at $x-\mathcal{V} t$. The function which assigns to $(t, x)$ the average value of $\gamma$ on the ray through $(t, x)$ is equal to $\widetilde{\gamma}(x-\mathcal{V} t)$. The function which subtracts from $\gamma(t, x)$ its average on the group line through $(t, x)$ is equal to $\gamma(t, x)-\widetilde{\gamma}(x-\mathcal{V} t)$.

Consider the solution $g$ of the scalar transport equation

$$
\left(\partial_{t}+\mathcal{V} . \partial_{x}\right) g=\gamma(t, x)-\widetilde{\gamma}(x-\mathcal{V} t),\left.\quad g\right|_{t=0}=0
$$

Then

$$
g(t, x)=\int_{0}^{t}(\gamma(s, x-\mathcal{V} t+\mathcal{V} s)-\widetilde{\gamma}(x-\mathcal{V} t)) d s
$$

Thus,

$$
\frac{g(t, x)}{t}=\frac{1}{t} \int_{0}^{t} \gamma(s, \widetilde{x}+\mathcal{V} s) d s-\widetilde{\gamma}(\widetilde{x}), \quad \widetilde{x}:=x-\mathcal{V} t
$$


Assumption (5.2) is equivalent to the fact that this is $o(1)$ as $t \rightarrow+\infty$,

$$
\lim _{t \rightarrow+\infty} \sup _{x \in \mathbb{R}^{N}} \frac{|g(t, x)|}{t}=0 .
$$

Equivalently, $g=o(t)$ as $t \rightarrow+\infty$.

Lemma 5.1. If $\gamma$ satisfies hypotheses (1.3) and (5.2) then,

i. each partial derivative $\partial_{t}^{j} \partial_{x}^{\beta} \gamma$ also satisfies the hypotheses,

ii. $\widetilde{\gamma} \in C^{\infty}\left(\mathbb{R}^{N}\right)$, and,

iii. for all $(j, \beta) \in \mathbb{N} \times \mathbb{N}^{N}$

$$
\lim _{T \rightarrow+\infty}\left\|\frac{1}{T} \int_{0}^{T} \partial_{t}^{j} \partial_{x}^{\beta} \gamma(t, x+\mathcal{V} t) d t-\left(-\mathcal{V} . \partial_{x}\right)^{j} \partial_{x}^{\beta} \widetilde{\gamma}(x)\right\|_{L^{\infty}\left(\mathbb{R}^{N}\right)}=0 .
$$

Proof. First treat the case of $x$ derivatives. Define

$$
G_{n}(x):=\frac{1}{n} \int_{0}^{n} \gamma(t, x+\mathcal{V} t) d t .
$$

Differentiating under the integral yields

$$
\partial_{x}^{\beta} G_{n}(x)=\frac{1}{n} \int_{0}^{n} \partial_{x}^{\beta} \gamma(t, x+\mathcal{V} t) d t
$$

Hypothesis (1.3) implies that for each $\beta$, the family $\left\{\partial_{x}^{\beta} G_{n}\right\}$ is bounded in $L^{\infty}\left(\mathbb{R}^{N}\right)$. Hypothesis (5.2) implies that $G_{n}$ converges uniformly to $\widetilde{\gamma}$ on $\mathbb{R}^{N}$. It follows that $\partial_{x}^{\beta} \widetilde{\gamma} \in$ $L^{\infty}\left(\mathbb{R}^{N}\right)$ and

$$
\lim _{n \rightarrow \infty}\left\|\partial_{x}^{\beta}\left(G_{n}-\widetilde{\gamma}\right)\right\|_{L^{\infty}\left(\mathbb{R}^{N}\right)}=0 .
$$

For $T>1$ choose $n$ to be the integer part of $T$. Then,

$$
\left\|\left(\frac{1}{T} \int_{0}^{T} \partial_{x}^{\beta} \gamma(t, x+\mathcal{V} t) d t-\partial_{x}^{\beta} G_{n}\right)\right\|_{L^{\infty}\left(\mathbb{R}^{N}\right)}=\mathcal{O}(1 / T) .
$$

Formula (5.5) for $j=0$ follows.

It remains to prove iii for $j>0$. This follows by induction from the case $j=1$. To prove the case $j=1$ use $\partial_{t} \gamma=\left(\partial_{t}+\mathcal{V} . \partial_{x}\right) \gamma-\mathcal{V} . \partial_{x} \gamma$ to find

$$
\left(\partial_{t} \gamma\right)(t, x+\mathcal{V} t)=\left(\frac{d}{d t}\right) \gamma(t, x+\mathcal{V} t)-\left(\mathcal{V} . \partial_{x} \gamma\right)(t, x+\mathcal{V} t)
$$

Integrating this equation on rays and using the case $j=0$ for the last term proves the case $j=1$.

We impose the following strengthening of (5.2) replacing the $o(t)$ by $O\left(t^{\beta}\right)$ for some $0 \leq \beta<1$. 
Definition 5.2. The function $\gamma$ satisfies the ray average hypothesis when (5.2) holds and there is a $0 \leq \beta<1$ so that for all $\alpha \in \mathbb{N} \times \mathbb{N}^{N}$ the solution $g_{\alpha}(t, x)$ of

$$
\left(\partial_{t}+\mathcal{V} . \partial_{x}\right) g_{\alpha}=\partial_{t, x}^{\alpha}(\gamma(t, x)-\widetilde{\gamma}(x-\mathcal{V} t)), \quad g_{\alpha}(0, x)=0
$$

satisfies $\langle t\rangle^{-\beta} g_{\alpha} \in L^{\infty}\left(\left[0, \infty\left[\times \mathbb{R}^{N}\right)\right.\right.$ where $\langle t\rangle:=\left(1+t^{2}\right)^{1 / 2}$.

Remark 5.3. i. For $\alpha=0$, we recover $g_{0}=g$, the solution of the transport equation (5.3). ii. The proof of Lemma 5.1 shows that if $\gamma$ satisfies the ray average hypothesis then so do its derivatives with the same value of $\beta$. iii. The proof of Lemma 5.1 shows that it suffices to treat the case $\alpha=0$. iv. The hypothesis is quite general. It takes a little ingenuity to construct examples that do not satisfy the hypothesis. For such an example the ideas of $\$ 5.1 .2$ are helpful. v. For random perturbations of periodic media one would expect analogous ray average hypotheses to hold with $\beta>0$.

\subsubsection{Examples of the ray average hypothesis with $\beta=0$.}

Proposition 5.4. i. The set of $\gamma$ satisfying the ray average hypothesis is a real vector space. It contains the functions satisfying (1.12).

ii. If $\gamma(t, x)=f(\ell(t, x))$ where $f(\theta)$ is a smooth periodic function of arbitrary period and $\ell$ is a linear functional then the ray average hypothesis is satisfied.

iii. If $\mathcal{M}: \mathbb{R}^{1+N} \rightarrow \mathbb{R}^{M}$ is linear and satisfies the small divisor hypothesis

$$
\exists C>0, m \in \mathbb{N}, \quad \forall n \in \mathbb{N}^{M}, \quad n . \mathcal{M}(1, \mathcal{V}) \neq 0 \Rightarrow \mid\left(n .\left.\mathcal{M}(1, \mathcal{V})|\geq C| n\right|^{-m}\right.
$$

then, for $h\left(\theta_{1}, \ldots, \theta_{M}\right) \in C^{\infty}\left(\mathbb{T}^{M}\right)$ the quasiperiodic function $\gamma(t, x)=h(\mathcal{M}(t, x))$ satisfies the hypothesis.

Proof. i. Self evident.

ii. Write the linear functional as $\alpha .(t, x)$ with $\alpha \in \mathbb{R}^{1+N}$. Let $\gamma=f \circ \ell$. Then,

$$
\gamma(t, x+\mathcal{V} t)=f(\alpha \cdot(t, x+\mathcal{V} t))=f(\alpha \cdot(0, x)+t \alpha \cdot(1, \mathcal{V}))
$$

There is a dichotomy. When $\alpha .(1, \mathcal{V})=0, \gamma$ is constant on group lines so $\gamma=\widetilde{\gamma}$ and $g=0$ so the hypothesis is satisfied.

When $\alpha .(1, \mathcal{V}) \neq 0$, the restriction of $\gamma$ to group lines is periodic with period $p /|\alpha .(1, \mathcal{V})|$ where $p$ is the period of $f$. Then $\gamma-\widetilde{\gamma}$ is periodic on group lines with the same period and has mean equal to zero. For any $t>0$ there is an $m \in \mathbb{N}$ so that

$$
m \frac{p}{|\alpha \cdot(1, \mathcal{V})|}<t \leq(m+1) \frac{p}{|\alpha \cdot(1, \mathcal{V})|}
$$


The interval $[0, m p /|\alpha .(1, \mathcal{V})|]$ is exactly equal to $m$ periods. Since the mean over one period is equal to zero, the integral over this interval of $m$ periods vanishes too. Therefore,

$$
\begin{aligned}
\left|\int_{0}^{t} \gamma(s, \widetilde{x}+\mathcal{V} s)-\widetilde{\gamma}(\widetilde{x}) d s\right| & =\left|\int_{m p /|\alpha \cdot(1, \mathcal{V})|}^{t} \gamma(s, \widetilde{x}+\mathcal{V} s)-\widetilde{\gamma}(\widetilde{x}) d s\right| \\
& \leq\|\gamma-\widetilde{\gamma}\|_{L^{\infty}} \frac{p}{|\alpha \cdot(1, \mathcal{V})|} \leq 2\|f\|_{L^{\infty}} \frac{p}{|\alpha \cdot(1, \mathcal{V})|} .
\end{aligned}
$$

This proves the boundedness of $g$. To prove the boundedness of derivatives, apply the above argument to the differentiated equation.

iii. For $\theta=\left(\theta_{1}, \ldots, \theta_{M}\right) \in \mathbb{T}^{M}$, express

$$
h=\sum_{n \in \mathbb{N}^{M}} h_{n} e^{i n \cdot \theta}, \quad \gamma=h \circ \mathcal{M}(t, x)=\sum_{n \in \mathbb{N}^{M}} h_{n} e^{i n \cdot \mathcal{M}(t, x)}:=\sum_{n \in \mathbb{N}^{M}} \gamma_{n}(t, x) .
$$

Along the ray $(t, x+\mathcal{V} t), \gamma_{n}$ is given by

$$
\gamma_{n}(t, x+\mathcal{V} t)=h_{n} e^{i n \cdot \mathcal{M}(0, x)} e^{i n \cdot \mathcal{M}(1, \mathcal{V}) t} .
$$

As in part $\mathbf{i}$, there is a dichotomy. If $n \cdot \mathcal{M}(1, \mathcal{V})=0$, then $\gamma_{n}$ is constant on rays so $\gamma_{n}-\widetilde{\gamma}_{n}=0$. Thus,

$$
\int_{0}^{t} \gamma(s, \widetilde{x}+\mathcal{V} s)-\widetilde{\gamma}(\widetilde{x}) d s=\sum_{n \cdot \mathcal{M}(1, \mathcal{V}) \neq 0} \int_{0}^{t} \gamma_{n}(s, \widetilde{x}+\mathcal{V} s)-\widetilde{\gamma}_{n}(\widetilde{x}) d s
$$

As in $(5.6),\left(\gamma_{n}-\widetilde{\gamma}_{n}\right)$ with $n . \mathcal{M}(1, \mathcal{V}) \neq 0$ is periodic with mean zero so,

$$
\begin{aligned}
\int_{0}^{t} \gamma_{n}(s, \widetilde{x}+\mathcal{V} s)-\widetilde{\gamma}_{n}(\widetilde{x}) d s & \leq\left\|\gamma_{n}-\widetilde{\gamma}_{n}\right\|_{L^{\infty}} \frac{2 \pi}{|n \cdot \mathcal{M}(1, \mathcal{V})|} \\
& \leq 2\left|h_{n}\right| \frac{2 \pi}{|n \cdot \mathcal{M}(1, \mathcal{V})|} \\
& \leq C_{K}|n|^{-K}|n|^{m},
\end{aligned}
$$

the last using rapid decrease and the small divisor hypothesis. Summing over $n . \mathcal{M}(1, \mathcal{V}) \neq$ 0 yields

$$
\left|\int_{0}^{t} \gamma(s, \widetilde{x}+\mathcal{V} s)-\widetilde{\gamma}(\widetilde{x}) d s\right| \leq \sum_{n \neq 0} C_{K}|n|^{-K}|n|^{m} .
$$

Choosing $K>N+1-m$ yields the $L^{\infty}\left(\mathbb{R}^{1+N}\right)$ bound for $g$. The bound for derivatives follows by applying the above argument to the differentiated equation using Lemma 5.1.

Proposition 5.5. i. If the components of $\mathcal{M}(1, \mathcal{V})$ have rational ratio, then the small divisor hypothesis is satisfied.

ii. If the dimension is $M=1+N$ and $\mathcal{M}=I$, then the small divisor hypothesis is satisfied for Lebesgue almost all $\mathcal{V}$. 
iii. If $\mathcal{V}$ and the dimension $M$ are fixed, then the small divisor hypothesis is satisfied for Lebesgue almost all $\mathcal{M}$.

iv. If the dimension $M$ is fixed, then the small divisor hypothesis is satisfied for Lebesgue almost all $\mathcal{M}, \mathcal{V}$.

Proof. i. The rational ratio is equivalent to the existence of an $r \in] 0, \infty[$ so that

$$
r \mathcal{M}(1, \mathcal{V})=\left(q_{0}, q_{1}, \ldots, q_{M}\right) \in \mathbb{Z}^{1+M}
$$

Then,

$$
\left(n_{0}, n_{1}, \ldots, n_{M}\right) \cdot \mathcal{M}(1, \mathcal{V})=\left(n_{0}, n_{1}, \ldots, n_{M}\right) \cdot \frac{1}{r}\left(q_{0}, q_{1}, \ldots, q_{M}\right) \in \frac{1}{r} \mathbb{Z} .
$$

When it is nonzero, it is equal to an integer divided by $r$ so is bounded below in absolute value by $1 / r$. This verifies the small divisor hypothesis with $C=1 / r$ and $m=0$.

ii. A vector $W$ satisfies the small divisor hypothesis if and only if

$$
\exists m \in \mathbb{N}, \quad \forall n \in \mathbb{N}^{N+1}, \quad n . W \neq 0 \Rightarrow|n . W| \geq \frac{1}{m|n|^{m}} .
$$

The set of vectors orthogonal to one of the $n$ is a null set. So it suffices to show that the complement of the set defined by

$$
\exists m \geq 0 \quad \forall n \neq 0, \quad|n . W| \geq \frac{1}{m|n|^{m}} .
$$

is a null set. A vector $W$ belongs to the complement if and only if

$$
\forall m \geq 0, \quad \exists n \neq 0, \quad|n . W|<\frac{|n|^{-m}}{m} .
$$

This is the set

$$
\bigcap_{m \geq 0} \bigcup_{n \neq 0}\left\{W:|n \cdot W|<\frac{|n|^{-m}}{m}\right\} .
$$

We show that the Lebesgue measure of this set is equal to zero, by showing that its intersection with $\{|W| \leq R\}$ is a null set. Toward that end, for each $n$, we rotate coordinates so that in the new coordinates, $n=(|n|, 0,0, \ldots, 0)$. Then

$$
\left\{W:|n \cdot W|<\frac{|n|^{-m}}{m}\right\}=\left\{\frac{|n \cdot W|}{|n|}<\frac{1}{m|n|^{m+1}}\right\} \subset\left\{\frac{|n \cdot W|}{|n|}<\frac{1}{m}\right\}
$$

The intersection of this set with the ball of radius $R$ has Lebesgue measure $\leq C(R) / m$. Since our set is the intersection on $m$ of such sets, it is a null set.

iii. Denote by $E \in \mathbb{R}^{N}$ the set of full Lebesgue measure so that the small divisor hypothesis is satisfied when $\mathcal{M}=I$ and $\mathcal{V} \in E$. Then, for $\mathcal{V}$ fixed, the pair $\mathcal{M}, \mathcal{V}$ satisfies the small divisor hypothesis whenever

$$
\mathcal{M}(1, \mathcal{V}) \in E
$$

This is satisfied for almost all $\mathcal{M}$. 
iv. Follows from iii and Fubini's Theorem.

5.1.2. Examples of the ray average hypothesis with $0<\beta<1$. The preceding section showed that quasiperiodic $\gamma$ satisfy the ray average hypothesis with $\beta=0$ under a small divisor hypothesis. In this section we show that much more general almost periodic $\gamma$ satisfy the hypothesis with $\beta>0$ under a weaker divisor hypothesis. The smooth almost periodic $\gamma$ are assumed to be of the form

$$
\gamma(t, x)=\sum_{\eta \in \mathbb{R}^{1+N}} a_{\eta} e^{i \eta \cdot(t, x)},
$$

where $a_{\eta}$ vanish for all but a countable family of $\eta$ and satisfy

$$
\forall n \in \mathbb{N}, \quad \sum_{\eta}\langle\eta\rangle^{n}\left|a_{\eta}\right|<\infty \quad\langle\eta\rangle:=\left(1+|\eta|^{2}\right)^{1 / 2} .
$$

Then

$$
\left(\partial_{t}+\mathcal{V} \partial_{x}\right) \gamma=\sum_{\eta \in \mathbb{R}^{1+N}} \eta \cdot(1, \mathcal{V}) a_{\eta} e^{i \eta \cdot(t, x)}, \quad \widetilde{\gamma}=\sum_{\eta \cdot(1, \mathcal{V})=0} a_{\eta} e^{i \eta \cdot(t, x)}
$$

Then

$$
\gamma(t, x)-\widetilde{\gamma}(x-\mathcal{V} t)=\sum_{\eta \cdot(1, \mathcal{V}) \neq 0} a_{\eta} e^{i \eta \cdot(t, x)} .
$$

The ray average of this difference vanishes but the convergence is slow for terms with $\eta \cdot(1, \mathcal{V})$ small. Lebesgue's theorem implies that

$$
\sum_{0<|\eta \cdot(1, \mathcal{V})|<\delta} a_{\eta} e^{i \eta \cdot(t, x)}=o(1) \quad \text { as } \quad \delta \rightarrow 0 .
$$

Our hypothesis strengthens this to $O\left(\delta^{\alpha}\right)$ for some $0<\alpha$.

Proposition 5.6. Suppose that $\gamma$ is as in (5.7), (5.8) and there is an $\alpha>0$ so that for all $n$

$$
\sum_{0<|\eta \cdot(1, \mathcal{V})|<\delta}\langle\eta\rangle^{n}\left|a_{\eta}\right|=O\left(\delta^{\alpha}\right), \quad \delta \rightarrow 0 .
$$

Then the ray average hypothesis of Definition 5.2 holds with $\beta=\alpha /(\alpha+1)$.

Proof. Consider the solution of $\left(\partial_{t}+V \partial_{x}\right) g=\gamma(t, x)-\widetilde{\gamma}(x-\mathcal{V} t)$ with vanishing initial data. Part iii of Remark 5.3 shows that it suffices to consider only $g$ and not its derivatives.

Write

$$
\gamma(t, x)-\widetilde{\gamma}(x-\mathcal{V} t)=\sum_{|\eta \cdot(1, \mathcal{V})|<\delta} a_{\eta} e^{i \eta \cdot(t, x)}+\sum_{|\eta \cdot(1, \mathcal{V})|>\delta} a_{\eta} e^{i \eta \cdot(t, x)}
$$

with corresponding solutions $g_{1}$ and $g_{2}$ with vanishing initial data. Estimate (5.9) shows that

$$
\left\|g_{1}\right\|_{C^{0}\left(\mathbb{R}^{N}\right)} \leq C \delta^{\alpha}
$$


The solution $g_{2}$ is given by

$$
g_{2}=\sum_{|\eta \cdot(1, \mathcal{V})|>\delta} \frac{1}{i \eta \cdot(1, V) t}\left(a_{\eta} e^{i \eta \cdot(t, x)}-a_{\eta} e^{i \eta \cdot(0, x-\mathcal{V} t)}\right) .
$$

The ray average hypothesis concerns only $t \geq 1$ and one has,

$$
\left\|g_{2}(t)\right\|_{C^{0}\left(\mathbb{R}^{N}\right)} \leq \frac{C}{t \delta}
$$

Choose $\delta$ so that $\delta^{\alpha}=1 /(t \delta)$, that is $\delta=t^{-1 /(1+\alpha)}$ to find that

$$
\left\|g_{1}(t)\right\|_{C^{0}\left(\mathbb{R}^{N}\right)}+\left\|g_{2}\right\|_{C^{0}\left(\mathbb{R}^{N}\right)} \leq \frac{C}{t^{\alpha /(\alpha+1)}} .
$$

5.2. The approximate solution. When the ray average hypothesis holds we construct a three term approximate solution

$$
\begin{gathered}
v^{\varepsilon}:=e^{2 \pi i S / \varepsilon} W^{\varepsilon}(\varepsilon t, t, x, x / \varepsilon), \\
W^{\varepsilon}(\mathcal{T}, t, x, y):=w_{0}(\mathcal{T}, t, x, y)+\varepsilon w_{1}(\mathcal{T}, t, x, y)+\varepsilon^{2} w_{2}(\mathcal{T}, t, x, y),
\end{gathered}
$$

with profiles $w_{j}$ smooth and $y$-periodic. The correctors $w_{1}$ and $w_{2}$ in this construction will not in general be bounded in $t$. The corrected solution does not have the form of infinitely accurate expansions constructed when (1.12) holds. The derivation of the leading approximation and the correctors follows the lines established in Section 4. The information gleaned from the leading residuals $r_{-2}, r_{-1}$ up to equation (4.14) is unchanged. Equation (4.15) is treated differently. In the case of coefficients satisfying the invariance (1.12), the equation was multiplied by $\partial_{t}+\mathcal{V} . \partial_{x}$ to eliminate $w_{0}$ and then to arrive at $\left(\partial_{t}+\mathcal{V} . \partial_{x}\right) \Pi w_{1}=0$. In the present case, we isolate the $w_{1}$ terms as

$$
(\Pi \mathbb{N} \Pi-\Pi \mathbb{M} Q \mathbb{M} \Pi) w_{0}=-\Pi \mathbb{M} \Pi w_{1}
$$

Propositions 4.1 and 3.2 (the latter modified for the diffractive case so that there is no $\gamma$ term) show that this is equivalent to,

$$
\left(4 \pi i \omega \partial_{\mathcal{T}}-\omega \nabla_{\theta}^{2} \omega\left(\partial_{x}, \partial_{x}\right)+\gamma(t, x)\right) w_{0}=-\left(\partial_{t}+\mathcal{V} . \partial_{x}\right) \Pi w_{1} .
$$

Split

$$
\gamma(t, x)=\widetilde{\gamma}(x-\mathcal{V} t)+(\gamma(t, x)-\widetilde{\gamma}(x-\mathcal{V} t))
$$

to write (5.14) as,

$\left(4 \pi i \omega \partial_{\mathcal{T}}-\omega \nabla_{\theta}^{2} \omega\left(\partial_{x}, \partial_{x}\right)+\widetilde{\gamma}(x-\mathcal{V} t)\right) w_{0}=-\left(\partial_{t}+\mathcal{V} . \partial_{x}\right) \Pi w_{1}-(\gamma(t, x)-\widetilde{\gamma}(x-\mathcal{V} t)) w_{0}$ 
The equation (5.15) is satisfied by first choosing $w_{0}(\mathcal{T}, t, x)=\widetilde{w}_{0}(\mathcal{T}, x-\mathcal{V} t)$ where $\widetilde{w}_{0}(\mathcal{T}, x)$ is a $\mathbb{K}$-valued function satisfying the Schrödinger equation

$$
\left(4 \pi i \omega \partial_{\mathcal{T}}-\omega \nabla_{\theta}^{2} \omega\left(\partial_{x}, \partial_{x}\right)+\widetilde{\gamma}(x)\right) \widetilde{w}_{0}=0,
$$

which implies that the left-hand side of (5.15) vanishes. The initial value, $\widetilde{w}_{0}(0, x) \in$ $\mathcal{S}\left(\mathbb{R}^{N} ; \mathbb{K}\right)$ is arbitrary. Once chosen, the unique solution satisfies for all $\alpha$,

$$
\left(x, \partial_{\mathcal{T}, t, x}\right)^{\alpha} w_{0} \in L^{\infty}\left([0, T]_{\mathcal{T}} \times\left[0, \infty\left[{ }_{t} \times \mathbb{R}_{x}^{N}\right) .\right.\right.
$$

The $\mathbb{K}$ valued function $\Pi w_{1}(t, x)$ is chosen as a solution of

$$
\left(\partial_{t}+\mathcal{V} . \partial_{x}\right) \Pi w_{1}=-(\gamma(t, x)-\widetilde{\gamma}(x-\mathcal{V} t)) w_{0}
$$

so the right-hand side of $(5.15)$ vanishes too. Since $w_{0}(\mathcal{T}, t, x)=\widetilde{w}_{0}(\mathcal{T}, x-\mathcal{V} t)$ is constant on group lines, the solution of the transport equation (5.18) can be chosen as,

$$
\Pi w_{1}(\mathcal{T}, t, x)=g(t, x) w_{0}(\mathcal{T}, t, x),
$$

where the scalar valued function $g$ is the solution of (5.3). The ray average hypothesis with parameter $0 \leq \beta<1$ yields estimates for the derivatives of $g$ and therefore those of $\Pi w_{1}$,

$$
\langle t\rangle^{-\beta}\left(x, \partial_{t, x}\right)^{\alpha}\left(\Pi w_{1}\right) \in L^{\infty}\left([0, T] \times[0, t] \times \mathbb{R}^{N} ; \mathbb{K}\right) .
$$

The component $(I-\Pi) w_{1}$ is given by (4.13) in terms of $w_{0}$ so (5.17) implies,

$$
\left(x, \partial_{T, t, x, y}\right)^{\alpha}(I-\Pi) w_{1} \in L^{\infty}\left([0, T] \times[0, t] \times \mathbb{R}_{x}^{N} \times \mathbb{R}_{y}^{N}\right),
$$

with $w_{1}$ is periodic in $y$. This completes the determination of $w_{0}$ and $w_{1}$. At this stage one has $r_{-2}=r_{-1}=\Pi r_{0}=0$.

We choose $w_{2}$ to that $(I-\Pi) r_{0}=0$. As earlier, the equation $(I-\Pi) r_{0}=0$ holds if and only if (4.27) is satisfied. This determines $(I-\Pi) w_{2}$. On the other hand, $\Pi w_{2}$ does not affect the profiles $r_{2}, r_{1}, r_{0}$. It is chosen equal to zero,

$$
\Pi w_{2}=0 .
$$

The estimates for $w_{0}, w_{1}$ imply that the $y$-periodic $w_{2}$ satisfies estimates analogous to those of $w_{1}$ so,

$$
\langle t\rangle^{-\beta}\left(x, \partial_{T, t, x, y}\right)^{\alpha} w_{j} \in L^{\infty}\left([0, T] \times[0, t] \times \mathbb{R}_{x}^{N} \times \mathbb{R}_{y}^{N}\right), \quad j=1,2 .
$$

This completes the determination of the profiles so that $r_{-2}=r_{-1}=r_{0}=0$.

Theorem 5.7. Suppose that the ray average hypothesis of Definition 5.2 is satisfied with $0 \leq \beta<1$ and that $\widetilde{w}_{0}(0, x)=\Pi \widetilde{w}_{0}(0, x) \in \mathcal{S}\left(\mathbb{R}^{N} ; \mathbb{K}\right)$ is chosen. The leading profile $w_{0}(\mathcal{T}, t, x)=\widetilde{w}_{0}(\mathcal{T}, x-\mathcal{V} t)$ satisfying $(5.17)$ is determined from the Schrödinger equation 
(5.16). Furthermore $w_{1}, w_{2}$ are determined from (5.18), (4.13), (4.27), (5.19), and satisfy (5.20). Then the approximate solution (5.12), (5.13) satisfies

$$
P^{\varepsilon}\left(t, x, \partial_{t, x}\right) v^{\varepsilon}=\varepsilon e^{2 \pi i S / \varepsilon} R^{\varepsilon}(\varepsilon t, t, x, x / \varepsilon),
$$

where $R^{\varepsilon}(T, t, x, y)$ is periodic in $y$ and for all $\left.\left.\alpha \in \mathbb{N}^{3 N+2}, T \in\right] 0, \infty\right]$,

$$
\left\|\langle t\rangle^{-\beta}\left(x, \partial_{T, t, x, y}\right)^{\alpha} R^{\varepsilon}(\mathcal{T}, t, x, y)\right\|_{L^{\infty}\left([0, T] \times\left[0, \infty\left[\times \mathbb{R}_{x}^{N} \times \mathbb{R}_{y}^{N}\right)\right.\right.} \leq C(\alpha),
$$

independent of $\varepsilon$.

The energy of the initial data of $v^{\varepsilon}$ is $\mathcal{O}(1 / \varepsilon)$ since the partial derivatives of first order are $\mathcal{O}(1 / \varepsilon)$. Denote by $u^{\varepsilon}$ the exact solution of the Cauchy problem (1.1) with the same initial data as $v^{\varepsilon}$,

$$
P^{\varepsilon}\left(t, x, \partial_{t, x}\right) u^{\varepsilon}=0,\left.\quad u^{\varepsilon}\right|_{t=0}=\left.v^{\varepsilon}\right|_{t=0},\left.\quad \partial_{t} u^{\varepsilon}\right|_{t=0}=\left.\partial_{t} v^{\varepsilon}\right|_{t=0} .
$$

Theorem 5.8. The functions $v^{\varepsilon}$ from Theorem 5.7 approximate the exact solutions $u^{\varepsilon}$ in (5.23) with relative error $\mathcal{O}\left(\varepsilon^{1-\beta}\right)$ in the sense that

$$
\exists C>0, \quad \sup _{0 \leq t \leq T / \varepsilon} \sup _{|\alpha| \leq 1}\left\|\left(\varepsilon \partial_{t, x}\right)^{\alpha}\left(u^{\varepsilon}-v^{\varepsilon}\right)\right\|_{L^{2}\left(\mathbb{R}^{N}\right)} \leq C \varepsilon^{1-\beta}, \quad \varepsilon \rightarrow 0 .
$$

Remark 5.9. The error in derivatives of higher order is not estimated. The previous cases had residual $\mathcal{O}\left(\varepsilon^{\infty}\right)$. Here the residual is $\mathcal{O}\left(\varepsilon^{1-\beta}\right)$ and no better. The possible unboundedness of the family $\left(P^{\varepsilon}\right)^{-1}$ as maps in higher Sobolev spaces (see §3.4) presents a serious obstruction.

Proof. Denote $w^{\varepsilon}:=u^{\varepsilon}-v^{\varepsilon}$. The error $w^{\varepsilon}$ is the solution of the Cauchy problem

$$
P^{\varepsilon} w^{\varepsilon}=-\varepsilon e^{2 \pi i S / \varepsilon} R^{\varepsilon}(\varepsilon t, t, x, x / \varepsilon),\left.\quad w^{\varepsilon}\right|_{t=0}=\left.\partial_{t} w^{\varepsilon}\right|_{t=0}=0 .
$$

The error estimate (5.24) with $|\alpha|=1$ is an immediate consequence of the classical energy estimate: $\forall T>0, \quad \exists C>0, \quad \forall 0<\varepsilon<1, \quad \forall 0 \leq t \leq T / \varepsilon$,

$$
\forall w \in C\left([0, T / \varepsilon] ; H^{1}\left(\mathbb{R}^{N}\right)\right) \cap C^{1}\left([0, T / \varepsilon] ; L^{2}\left(\mathbb{R}^{N}\right)\right),
$$

with $P^{\varepsilon} w \in L^{1}\left([0, T / \varepsilon] ; L^{2}\left(\mathbb{R}^{N}\right)\right)$,

$$
\sum_{|\alpha|=1}\left\|\partial_{t, x}^{\alpha} w(t)\right\|_{L^{2}\left(\mathbb{R}^{N}\right)} \leq C\left(\sum_{|\alpha|=1}\left\|\partial_{t, x}^{\alpha} w(0)\right\|_{L^{2}\left(\mathbb{R}^{N}\right)}+\int_{0}^{t}\left\|P^{\varepsilon} w(s)\right\|_{L^{2}\left(\mathbb{R}^{N}\right)} d s\right) .
$$

Using (5.21), (5.22) the last term on the right is bounded by $\mathcal{O}\left(\langle t\rangle^{-\beta} \varepsilon\right)=\mathcal{O}\left(\varepsilon^{1-\beta}\right)$. This is the desired estimate for $|\alpha|=1$.

The error estimate (5.24) for $\alpha=0$ is subtle and occupies the remainder of this subsection. In contrast with the case $|\alpha|=1$, it uses in an essential way the oscillations in $t$ of the right hand side of (5.25). In the case of $\S 3.4$ estimates for $u^{\varepsilon}$ are recovered from estimates for $u_{t}^{\varepsilon}$ by integrating in time. In the present context the integration is over an 
interval of length $\sim 1 / \varepsilon$ and oscillations in time are used to show that the integral is not large.

The proof of Lemma 5.12 below uses an important symmetry. Suppress the $\varepsilon$ in the operator $P^{\varepsilon}\left(t, x, \partial_{t}, \partial_{x}\right)$. The transposed operator $P^{\dagger}$ is given by,

$$
P^{\dagger}\left(\partial_{t}, \partial_{x}\right) w:=\partial_{t}^{2}(\rho w)-\operatorname{div}_{x}\left(A \operatorname{grad}_{x} w\right) .
$$

Introduce the linear map $P^{-1}: C_{0}^{\infty}\left(\mathbb{R}^{1+N}\right) \rightarrow C^{\infty}\left(\mathbb{R}^{1+N}\right)$ by $u=P^{-1}(f)$ is the unique solution of $P u=f$ such that if $f$ vanishes for $t<\underline{t}$ then so does $u$. The function $u$ is the solution of the forward wave problem with source $f$. Similarly, for $g \in C_{0}^{\infty}\left(\mathbb{R}^{1+N}\right)$, $w=\left(P^{\dagger}\right)^{-1} g$ is the unique solution of $P^{\dagger} w=g$ so that if $g$ vanishes for $t>\underline{t}$, then so does $w$. The function $w$ solves the backward radiation problem for $P^{\dagger}$ with source $g$.

Denote by $K(t, x, s, y)$ and $K^{\dagger}(t, x, s, y)$ the Schwartz kernels of the operators $P^{-1}$ and $\left(P^{\dagger}\right)^{-1}$ respectively so that with the usual abuse of notation,

$$
\left(P^{-1} f\right)(t, x)=\int_{\mathbb{R}^{1+N}} K(t, x, s, y) f(s, y) d s d y, \quad\left(P^{\dagger}\right)^{-1} g(t, x)=\int_{\mathbb{R}^{1+N}} K^{\dagger}(t, x, s, y) g(s, y) d s d y
$$

The precise version is that for all $f, g \in C_{0}^{\infty}\left(\mathbb{R}^{1+N}\right)$

$$
\int g(s, y)\left(P^{-1} f\right)(s, y) d t d x d s d y=\langle K, f(t, x) g(s, y)\rangle
$$

with an analogous expression for $\left(P^{\dagger}\right)^{-1}$ and $K^{\dagger}$. Here $\langle$,$\rangle denotes the pairing be-$ tween compactly supported test functions and the distributions on $\mathbb{R}_{t, x}^{1+N} \times \mathbb{R}_{s, y}^{1+N}$. The distribution $K$ and $K^{\dagger}$ are for $N>2$ not locally integrable functions.

The kernel $K$ is determined by the following recipe. For $s, y$ fixed

$$
P\left(t, x, \partial_{t}, \partial_{x}\right) K(t, x, s, y)=\delta_{s, y}, \quad K=0 \quad \text { when } \quad t<s .
$$

Similarly, the kernel $K^{\dagger}$ is determined as follows. For $t, x$ fixed

$$
P^{\dagger}\left(s, y, \partial_{s}, \partial_{y}\right) K^{\dagger}(t, x, s, y)=\delta_{t, x}, \quad K^{\dagger}=0 \quad \text { when } \quad s<t .
$$

The next reciprocity lemma is analogous to the fact that for a matrix, the inverse of the transpose is equal to the transpose of the inverse. The proof of the lemma is modelled on the matrix case.

Lemma 5.10. The kernels $K(t, x, s, y)$ and $K^{\dagger}(t, x, s, y)$ satisfy the reciprocity relation,

$$
K(t, x, s, y)=K^{\dagger}(s, y, t, x) .
$$

Proof. The assertion is equivalent to the identity, $\forall f, g \in C_{0}^{\infty}\left(\mathbb{R}^{1+N}\right)$,

$$
\langle K, g(t, x) f(s, y)\rangle_{\mathbb{R}^{1+N} \times \mathbb{R}^{1+N}}=\left\langle K^{\dagger}, g(s, y) f(t, x)\right\rangle_{\mathbb{R}^{1+N} \times \mathbb{R}^{1+N}} .
$$

The left hand side of (5.27) is equal to

$$
\text { l.h.s. }=\left\langle P^{-1} f, g\right\rangle_{\mathbb{R}^{1+N}}=\left\langle P^{-1} f, P^{\dagger} w\right\rangle_{\mathbb{R}^{1+N}} .
$$


Integrating by parts yields

$$
\text { l.h.s. }=\left\langle P P^{-1} f, w\right\rangle_{\mathbb{R}^{1+N}}=\langle f, w\rangle_{\mathbb{R}^{1+N}}=\left\langle f,\left(P^{\dagger}\right)^{-1} g\right\rangle_{\mathbb{R}^{1+N}} .
$$

The last expression is equal to the right hand side of (5.27).

Duhamel's formula gives the following expression for $K(t, x, s, y)$. Define $G(t, x, s, y)$ to be the unique solution of

$$
P\left(t, x, \partial_{t}, \partial_{x}\right) G=0,\left.\quad G\right|_{t=s}=0,\left.\quad\left(\partial_{t} G\right)\right|_{t=s}=\frac{1}{\rho} \delta_{y} .
$$

Then

$$
K(t, x, s, y)= \begin{cases}G & \text { when } t \geq s \\ 0 & \text { when } t \leq s\end{cases}
$$

At the transition points where $\{t=s\}, G=0$. Therefore,

$$
\partial_{s} K(t, x, s, y)=\left\{\begin{array}{ccc}
\partial_{s} G & \text { when } t \geq s \\
0 & \text { when } t \leq s .
\end{array}\right.
$$

We need an estimate for $\left\|\int \partial_{s} K(t, x, s, y) \phi(y) d y\right\|_{L^{2}\left(\mathbb{R}^{N}\right)}$. The estimate uses a preliminary lemma asserting that the evolution defined by the transposed operator is bounded on time intervals of length $O(1 / \varepsilon)$.

Lemma 5.11. For any $T>0$, there is a constant $C>0$ so that for all $t, s, \varepsilon$ satisfying $|t-s|<T / \varepsilon$ and

$$
w \in C\left([\min (t, s), \max (t, s)] ; H^{1}\left(\mathbb{R}^{N}\right)\right) \cap C^{1}\left([\min (t, s), \max (t, s)] ; L^{2}\left(\mathbb{R}^{N}\right)\right)
$$

with

$$
P^{\dagger} w \in L^{1}\left([\min (t, s), \max (t, s)] ; L^{2}\left(\mathbb{R}^{N}\right)\right)
$$

one has

$$
\begin{aligned}
& \left\|\partial_{t, x} w(t)\right\|_{L^{2}\left(\mathbb{R}^{N}\right)}+\varepsilon\|w(t)\|_{L^{2}\left(\mathbb{R}^{N}\right)} \leq \\
& \quad C\left(\left\|\partial_{t, x} w(s)\right\|_{L^{2}\left(\mathbb{R}^{N}\right)}+\varepsilon\|w(s)\|_{L^{2}\left(\mathbb{R}^{N}\right)}+\left|\int_{s}^{t}\left\|P^{\dagger} w(\sigma)\right\|_{L^{2}\left(\mathbb{R}^{N}\right)} d \sigma\right|\right) .
\end{aligned}
$$

Proof. Replacing $w$ by a cutoff and smoothed $w^{\varepsilon}$ then passing to the limit $\varepsilon \rightarrow 0$ shows that it suffices to consider real solutions which are smooth and rapidly decreasing as $x \rightarrow \infty$. For such solutions compute,

$$
\begin{gathered}
\int_{\mathbb{R}^{N}} \partial_{t} w P^{\dagger} w d x=\int_{\mathbb{R}^{N}} \partial_{t} w \partial_{t}^{2}(\rho w)+\left\langle\partial_{x} \partial_{t} w, A \partial_{x} w\right\rangle d x \\
\int_{\mathbb{R}^{N}}\left\langle\partial_{x} \partial_{t} w, A \partial_{x} w\right\rangle d x=\frac{1}{2} \frac{d}{d t} \int_{\mathbb{R}^{N}}\left\langle\partial_{x} w, A \partial_{x} w\right\rangle d x-\frac{1}{2} \int_{\mathbb{R}^{N}}\left\langle\partial_{x} w, \partial_{t} A \partial_{x} w\right\rangle d x \\
\partial_{t}\left(\frac{\rho\left(\partial_{t} w\right)^{2}}{2}\right)=\frac{1}{2} \partial_{t} \rho\left(\partial_{t} w\right)^{2}+\rho \partial_{t} w \partial_{t}^{2} w
\end{gathered}
$$


where $\partial_{x}$ denotes the operator grad, and,

$$
\begin{aligned}
\partial_{t} w \partial_{t}^{2}(\rho w) & =\partial_{t} w\left(w \partial_{t}^{2} \rho+2 \partial_{t} \rho \partial_{t} w+\rho \partial_{t}^{2} w\right) \\
& =\partial_{t}\left(\frac{\rho\left(\partial_{t} w\right)^{2}}{2}\right)+\frac{3}{2} \partial_{t} \rho\left(\partial_{t} w\right)^{2}+w \partial_{t}^{2} \rho \partial_{t} w
\end{aligned}
$$

Since the unperturbed periodic medium is time independent, the coefficients $\partial_{t} A, \partial_{t} \rho, \partial_{t}^{2} \rho$ involve only the $\mathcal{O}\left(\varepsilon^{2}\right)$ perturbations so are $O\left(\varepsilon^{2}\right)$.

Introduce the energy

$$
E^{2}(t):=\frac{1}{2} \int_{\mathbb{R}^{N}}\left(\rho\left(\partial_{t} w\right)^{2}(t)+\left\langle\partial_{x} w(t), A \partial_{x} w(t)\right\rangle\right) d x .
$$

The preceding computations show that

$$
\left|\partial_{t} E^{2}-\int_{\mathbb{R}^{N}} \partial_{t} w P^{\dagger} w d x\right| \leq C\left(\varepsilon^{2} E^{2}+\varepsilon^{2} E\|w\|_{L^{2}\left(\mathbb{R}^{N}\right)}\right) .
$$

Estimating the integral by the Cauchy-Schwarz inequality yields,

$$
\left|\partial_{t} E^{2}\right| \leq C\left(E\left\|P^{\dagger} w\right\|_{L^{2}\left(\mathbb{R}^{N}\right)}+\varepsilon^{2} E^{2}+\varepsilon^{2} E\|w\|_{L^{2}\left(\mathbb{R}^{N}\right)}\right) .
$$

Since $\partial_{t} E^{2}=2 E \partial_{t} E$ this yields

$$
\left|\partial_{t} E\right| \leq C\left(\left\|P^{\dagger} w\right\|_{L^{2}\left(\mathbb{R}^{N}\right)}+\varepsilon^{2} E+\varepsilon^{2}\|w\|_{L^{2}\left(\mathbb{R}^{N}\right)}\right) .
$$

Complementing this estimate is

$$
\left|\partial_{t}\left(\|w(t)\|_{L^{2}\left(\mathbb{R}^{N}\right)}^{2}\right)\right|=\left|2 \int_{\mathbb{R}^{N}} \partial_{t} w w d x\right| \leq 2\left\|\partial_{t} w(t)\right\|_{L^{2}\left(\mathbb{R}^{N}\right)}\|w(t)\|_{L^{2}\left(\mathbb{R}^{N}\right)} \leq C E\|w(t)\|_{L^{2}\left(\mathbb{R}^{N}\right)} .
$$

Since $\partial_{t}\left(\|w(t)\|_{L^{2}\left(\mathbb{R}^{N}\right)}^{2}\right)=2\|w(t)\|_{L^{2}\left(\mathbb{R}^{N}\right)} \partial_{t}\|w(t)\|_{L^{2}\left(\mathbb{R}^{N}\right)}$, this implies,

$$
\left|\partial_{t}\|w(t)\|_{L^{2}\left(\mathbb{R}^{N}\right)}\right| \leq C E .
$$

Adding yields

$$
\left|\partial_{t}\left(E+\varepsilon\|w(t)\|_{L^{2}\left(\mathbb{R}^{N}\right)}\right)\right| \leq C\left(\left\|P^{\dagger} w\right\|_{L^{2}\left(\mathbb{R}^{N}\right)}+\varepsilon\left(E+\varepsilon\|w(t)\|_{L^{2}\left(\mathbb{R}^{N}\right)}\right)\right) .
$$

Integrating this yields

$$
E(t)+\varepsilon\|w(t)\|_{L^{2}\left(\mathbb{R}^{N}\right)} \leq C e^{C \varepsilon|t-s|}\left(E(s)+\varepsilon\|w(s)\|_{L^{2}\left(\mathbb{R}^{N}\right)}\right)+C e^{C \varepsilon|t-s|}\left|\int_{s}^{t}\left\|P^{\dagger} w(\sigma)\right\|_{L^{2}\left(\mathbb{R}^{N}\right)} d \sigma\right| .
$$

Since $E(t)+\varepsilon\|w(t)\|_{L^{2}\left(\mathbb{R}^{N}\right)}$ is a family of norms uniformly equivalent to the norms $\left\|\partial_{t, x} w\right\|_{L^{2}\left(\mathbb{R}^{N}\right)}+\varepsilon\|w(t)\|_{L^{2}\left(\mathbb{R}^{N}\right)}$, this completes the proof of (5.28) and therefore of Lemma 5.11 . 
For $t \geq s$ introduce the operator $K(t, s)$ whose kernel is $K(t, x, s, y)$. With the usual abusive use of an integral sign for the operator with a given distribution kernel, for $\phi \in C_{0}^{\infty}\left(\mathbb{R}^{N}\right)$

$$
(K(t, s) \phi)(x):=\int_{\mathbb{R}^{N}} K(t, x, s, y) \phi(y) d y,
$$

as well as its derivative with respect to $s$

$$
\left(\partial_{s} K(t, s) \phi\right)(x):=\int_{\mathbb{R}^{N}} \partial_{s} K(t, x, s, y) \phi(y) d y .
$$

The precise version is that for $\psi, \phi \in C_{0}^{\infty}\left(\mathbb{R}^{1+N}\right)$,

$$
\int \psi(x)(K(t, s) \phi)(x) d x=\langle K(t, x, s, y), \psi(x) \phi(y)\rangle_{\mathbb{R}_{x}^{N} \times \mathbb{R}_{y}^{n}} .
$$

Lemma 5.12. For all $T$ and $s \leq t$ with $t-s \leq T / \varepsilon$ there is a constant $C$ so that the following operator estimates hold,

$$
\begin{gathered}
\|K(t, s) \phi\|_{L^{2}\left(\mathbb{R}^{N}\right)} \leq C(t-s)\|\phi\|_{L^{2}\left(\mathbb{R}^{N}\right)}, \\
\left\|\partial_{s} K(t, s) \phi\right\|_{L^{2}\left(\mathbb{R}^{N}\right)} \leq C\|\phi\|_{L^{2}\left(\mathbb{R}^{N}\right)} .
\end{gathered}
$$

Proof. With $s$ fixed, the function $z(t, x)=\int_{\mathbb{R}^{N}} K(t, x, s, y) \phi(y) d y$ is uniquely determined for $t \geq s$ by

$$
P\left(t, x, \partial_{t}, \partial_{x}\right) z=0,\left.\quad z\right|_{t=s}=0,\left.\quad \partial_{t} z\right|_{t=s}=\phi / \rho .
$$

The energy estimate shows that

$$
\left\|\partial_{t} z\right\|_{L^{2}\left(\mathbb{R}^{N}\right)} \leq C\|\phi\|_{L^{2}\left(\mathbb{R}^{N}\right)} \quad \text { provided } \quad t-s<T / \varepsilon .
$$

Writing $z(t)=\int_{s}^{t} \partial_{t} z(\sigma) d \sigma$ yields

$$
\|z(t)\|_{L^{2}\left(\mathbb{R}^{N}\right)} \leq C(t-s)\|\phi\|_{L^{2}\left(\mathbb{R}^{N}\right)} .
$$

This is exactly (5.29).

To prove (5.30) reason by duality. Inequality (5.30) is equivalent to

$$
\left|\left\langle\partial_{s} K(t, x, s, y) \phi(y) \psi(x)\right\rangle_{\mathbb{R}_{x}^{N} \times \mathbb{R}_{y}^{N}}\right| \leq C\|\phi\|_{L^{2}\left(\mathbb{R}^{N}\right)}\|\psi\|_{L^{2}\left(\mathbb{R}^{N}\right)} .
$$

With $t$ fixed, define by the formal expression $\zeta(s, y):=\int K(t, x, s, y) \psi(x) d x$. Precisely

$$
\langle\zeta(t, y), \phi(x)\rangle:=\langle K(t, x, s, y), \phi(x) \psi(y)\rangle \text {. }
$$

Lemma 5.10 implies that for $s \leq t$ the function $\zeta$ is the solution of,

$$
P^{\dagger}\left(s, y, \partial_{s}, \partial_{y}\right) \zeta=0,\left.\quad \zeta\right|_{s=t}=0,\left.\quad \partial_{s} \zeta\right|_{s=t}=\frac{\psi(y)}{\rho(s, y)} .
$$


Since $t-s \leq T / \varepsilon$, the energy estimate for $P^{\dagger}$ proved in Lemma 5.11 yields the following estimate for $\partial_{s} \zeta(s)$,

$$
\left\|\partial_{s} \zeta(s)\right\|_{L^{2}\left(\mathbb{R}^{N}\right)} \leq C\|\psi\|_{L^{2}\left(\mathbb{R}^{N}\right)} .
$$

The Cauchy-Schwartz inequality implies

$\left|\left\langle\partial_{s} K(t, x, s, y), \phi(x) \psi(y)\right\rangle\right|=\left|\left\langle\partial_{s} \zeta, \phi\right\rangle\right| \leq\left\|\partial_{s} \zeta(s)\right\|_{L^{2}\left(\mathbb{R}^{N}\right)}\|\phi\|_{L^{2}\left(\mathbb{R}^{N}\right)} \leq C\|\psi\|_{L^{2}\left(\mathbb{R}^{N}\right)}\|\phi\|_{L^{2}\left(\mathbb{R}^{N}\right)}$.

This proves (5.31) and therefore the equivalent (5.30).

Lemma 5.12 is now used to prove the $L^{2}$ estimate corresponding to the case $\alpha=0$ in (5.24) and therefore complete the proof of Theorem 5.8. The solution of (5.25) is

$$
w^{\varepsilon}(t, x)=-\varepsilon \int_{0}^{t} K(t, s) e^{2 \pi i\left(\omega s+\theta_{0} \cdot x\right) / \varepsilon} R^{\varepsilon}(\varepsilon s, s, x, x / \varepsilon) d s .
$$

For ease of reading introduce

$$
r^{\varepsilon}(\mathcal{T}, t, x, y):=e^{2 \pi i \theta_{0} . y} R^{\varepsilon}(\mathcal{T}, t, x, y) .
$$

which is $\theta_{0}$-periodic in $y$. It inherits from $R^{\varepsilon}$ the bounds (5.22)

$$
\left\|\langle t\rangle^{-\beta}\left(x, \partial_{\mathcal{T}, t, x, y}\right)^{\alpha} r^{\varepsilon}(\mathcal{T}, t, x, y)\right\|_{L^{\infty}\left([0, T] \times\left[0, \infty\left[\times \mathbb{R}_{x}^{N} \times \mathbb{R}_{y}^{N}\right)\right.\right.} \leq C,
$$

independent of $\varepsilon$. Preparing for an integration by parts write,

$$
w^{\varepsilon}=\frac{-\varepsilon^{2}}{2 \pi i \omega} \int_{0}^{t} K(t, s) r^{\varepsilon}(\varepsilon s, s, x, x / \varepsilon) \partial_{s} e^{2 \pi i \omega s / \varepsilon} d s .
$$

Integration by parts yields,

$$
\begin{gathered}
w^{\varepsilon}=\frac{\varepsilon^{2}}{2 \pi i \omega} \int_{0}^{t}\left(\partial_{s} K(t, s) r^{\varepsilon}(\varepsilon s, s, x, x / \varepsilon)+K(t, s)\left(\varepsilon \partial_{\mathcal{T}} r^{\varepsilon}+\partial_{t} r^{\varepsilon}\right)(\varepsilon s, s, x, x / \varepsilon)\right) e^{2 \pi i \omega s / \varepsilon} d s \\
\quad-\left.\frac{\varepsilon^{2}}{2 \pi i \omega} K(t, s) r^{\varepsilon}(\varepsilon s, s, x, x / \varepsilon) e^{2 \pi \omega i s / \varepsilon}\right|_{s=0} ^{s=t} .
\end{gathered}
$$

We must show that $\left\|w^{\varepsilon}(t)\right\|_{L^{2}\left(\mathbb{R}^{N}\right)} \leq C \varepsilon^{1-\beta}$ independent of $0<t<T / \varepsilon$ and $\varepsilon$. There are two summands in the integral and two boundary terms.

Begin with the boundary terms. Since $K(t, t)=0$, one of the terms vanishes. Because of (5.29), the $L^{2}$ norm of the other is bounded by $C \varepsilon^{2-\beta}|t|$. Since $|t| \leq T / \varepsilon$ this yields the desired $\mathcal{O}\left(\varepsilon^{1-\beta}\right)$ bound.

For the $\partial_{s} K$ summand, (5.30) suffices to give the $\mathcal{O}\left(\varepsilon^{1-\beta}\right)$ estimate.

There remains only one term to estimate,

$$
\frac{\varepsilon^{2}}{2 \pi i \omega} \int_{0}^{t} K(t, s)\left(\varepsilon \partial_{\mathcal{T}} r^{\varepsilon}+\partial_{t} r^{\varepsilon}\right)(\varepsilon s, s, x, x / \varepsilon) e^{2 \pi i \omega s / \varepsilon} d s .
$$


This term is dangerous because $K(t, s)$ can be of size $\sim T / \varepsilon$ on an interval of size $\sim T / \varepsilon$ which might compensate the prefactor of $\varepsilon^{2}$. To get a better estimate, integrate by parts once more to find,

$$
\begin{aligned}
\frac{\varepsilon^{2}}{2 \pi i \omega} \int_{0}^{t} K(t, s) & \left(\varepsilon \partial_{\mathcal{T}}+\partial_{t}\right) r^{\varepsilon}(\varepsilon s, s, x, x / \varepsilon) \frac{\varepsilon}{2 \pi i \omega} \frac{d}{d s} e^{2 \pi i \omega s / \varepsilon} d s \\
= & \frac{-\varepsilon^{3}}{(2 \pi i \omega)^{2}} \int_{0}^{t} \frac{d}{d s}\left(K(t, s)\left(\varepsilon \partial_{\mathcal{T}}+\partial_{t}\right) r^{\varepsilon}(\varepsilon s, s, x, x / \varepsilon)\right) e^{2 \pi i \omega s / \varepsilon} d s \\
& +\left.\frac{\varepsilon^{3}}{(2 \pi i \omega)^{2}} K(t, s)\left(\varepsilon \partial_{\mathcal{T}}+\partial_{t}\right) r^{\varepsilon}(\varepsilon s, s, x, x / \varepsilon) e^{2 \pi i \omega s / \varepsilon}\right|_{s=0} ^{s=t} .
\end{aligned}
$$

The integrand of the first term on the right is equal to

$$
\partial_{s} K(t, s)\left(\varepsilon \partial_{\mathcal{T}}+\partial_{t}\right) r^{\varepsilon} e^{2 \pi i \omega s / \varepsilon}+K(t, s)\left(\varepsilon \partial_{\mathcal{T}}+\partial_{t}\right)^{2} r^{\varepsilon} e^{2 \pi i \omega s / \varepsilon} .
$$

Using (5.22), the first term has $L^{2}$ norm $\mathcal{O}\left(t^{\beta}\right)$ while the second term has $L^{2}$ norm bounded by $\mathcal{O}\left(t^{\beta}|t-s|\right)$. So the $L^{2}$ norm of the sum, when integrated with respect to $s$ over an interval no longer than $T / \varepsilon$, is bounded by $C T^{2} / \varepsilon^{2+\beta}$. The prefactor $\varepsilon^{3}$ yields the desired $\mathcal{O}\left(\varepsilon^{1-\beta}\right)$ bound. The boundary term at $s=t$ vanishes and that at $s=0$ has $L^{2}$ norm less than $C \varepsilon^{2-\beta}$ since $\|K(t, 0)\| \leq C T / \varepsilon$ from (5.29). This completes the proof of Theorem 5.8 .

5.3. The oscillatory initial value problem. Using two approximate solutions from Section 5.2, we find an approximate solution of the oscillatory initial value problem,

$$
\begin{aligned}
& P^{\varepsilon} u^{\varepsilon}=0, \\
& u^{\varepsilon}(0, x)=b(x) e^{2 \pi i x \cdot \theta_{0} / \varepsilon} \psi_{n}(x / \varepsilon), \\
& \partial_{t} u^{\varepsilon}(0, x)=\frac{c(x)}{\varepsilon} e^{2 \pi i x \cdot \theta_{0} / \varepsilon} \psi_{n}(x / \varepsilon),
\end{aligned}
$$

with $b, c \in \mathcal{S}\left(\mathbb{R}^{N}\right)$. There are two linear phases $S^{ \pm}$with $S^{ \pm}(0, x)=\theta_{0} \cdot x$ at $t=0$,

$$
S^{+}(t, x)=\theta_{0} \cdot x+\omega t \quad \text { and } \quad S^{-}(t, x)=\theta_{0} \cdot x-\omega t
$$

with associated group velocities $\pm \mathcal{V}$. Similarly we denote by $\widetilde{\gamma}^{ \pm}$the two averaged zeroorder (potential) terms in the limit Schrödinger equation (5.16) corresponding to the two velocities $\pm \mathcal{V}$.

Theorem 5.13. Assume that the ray average hypothesis in Definition 5.2 with parameter $0 \leq \beta<1$ is satisfied for both group velocities $\pm \mathcal{V}$. The solution $u^{\varepsilon}(t, x)$ of the initial value problem (5.33) admits the following approximation

$$
\sum_{ \pm} v^{\varepsilon, \pm}:=\sum_{ \pm} e^{2 \pi i S^{ \pm} / \varepsilon} \widetilde{W}^{\varepsilon, \pm}(\varepsilon t, x \mp \mathcal{V} t, x / \varepsilon)
$$


with

$$
\widetilde{W}^{\varepsilon, \pm}(\mathcal{T}, x, y)=\sum_{j=0}^{2} \varepsilon^{j} \widetilde{w}_{j}^{ \pm}(\mathcal{T}, x, y) \quad \widetilde{w}_{0}^{ \pm}(\mathcal{T}, x, y)=\widetilde{a}_{0}^{ \pm}(\mathcal{T}, x) \psi_{n}(y) .
$$

The initial data $\widetilde{w}_{0}^{ \pm}(0, x)$ are determined by

$$
\widetilde{a}_{0}^{+}(0, x)+\widetilde{a}_{0}^{-}(0, x)=b(x), \quad \widetilde{a}_{0}^{+}(0, x)-\widetilde{a}_{0}^{-}(0, x)=\frac{c(x)}{2 \pi i \omega},
$$

so that the initial data of the exact and approximate solutions differ by $\mathcal{O}(\varepsilon)$ in the sense that (5.35) holds for $T=0$. The profiles $\widetilde{w}_{j}^{ \pm}(\mathcal{T}, x, y)$ are determined from the equations of section 5.2 (with the corresponding zero-order term $\widetilde{\gamma}^{ \pm}$). Then for any $T>0$, there is a constant $C>0$ so that

$$
\sup _{0 \leq t \leq T / \varepsilon} \sup _{|\alpha| \leq 1}\left\|\left(\varepsilon \partial_{t, x}\right)^{\alpha}\left(u^{\varepsilon}-\left(v^{\varepsilon,+}+v^{\varepsilon,-}\right)\right)\right\|_{L^{2}\left(\mathbb{R}^{N}\right)} \leq C \varepsilon^{1-\beta} .
$$

Proof. The proof is like that of Theorem 5.8.

Remark 5.14. In both Theorems 5.8 and 5.13 one can replace the three term approximate solutions $v^{\varepsilon, \pm}$ by their leading term $e^{2 \pi i S / \varepsilon} \widetilde{w}_{0}(\varepsilon t, x-\mathcal{V} t, x / \varepsilon)$ or $e^{2 \pi i S^{ \pm} / \varepsilon} \widetilde{w}_{0}^{ \pm}(\varepsilon t, x \mp \mathcal{V} t, x / \varepsilon)$. Indeed, the two other terms involving $w_{1}$ and $w_{2}$ are smaller by a factor of $\varepsilon^{1-\beta}$ in the norms of (5.35). This simplification is made in the statement of Theorem 1.3. The corrector terms are crucial in the proof, as is usual in asymptotic analysis.

5.4. Diffractive geometric optics with $H^{1}$ amplitudes. The leading term of the approximate solutions of diffractive geometric optics is given by

$$
v_{\text {approx }}^{\varepsilon}=e^{2 \pi i S / \varepsilon} \psi_{n}\left(x / \varepsilon, \theta_{0}\right) a(\varepsilon t, x-\mathcal{V} t)
$$

with $a(\mathcal{T}, x)$ satisfying the Schrödinger equation

$$
\left(4 \pi i \omega \partial_{\mathcal{T}}-\omega \nabla_{\theta}^{2} \omega\left(\partial_{x}, \partial_{x}\right)+\widetilde{\gamma}(x)\right) a=0
$$

The Cauchy data of $v_{\text {approx }}^{\varepsilon}$ are equal to

$$
\begin{aligned}
& v_{\text {approx }}^{\varepsilon}(0, x)=e^{2 \pi i \theta_{0} \cdot x / \varepsilon} \psi_{n}\left(x / \varepsilon, \theta_{0}\right) a(0, x), \\
& \partial_{t} v_{\text {approx }}^{\varepsilon}(0, x)=\frac{2 \pi i \omega}{\varepsilon} e^{2 \pi i \theta_{0} \cdot x / \varepsilon} \psi_{n}\left(x / \varepsilon, \theta_{0}\right) a(0, x)+\mathcal{O}(1) .
\end{aligned}
$$

In this section we explain how the analysis when $a(0, \cdot) \in \mathcal{S}\left(\mathbb{R}^{N}\right)$ suffices to justify the approximation of diffractive geometric optics when $a(0, \cdot) \in H^{1}\left(\mathbb{R}^{N}\right)$.

The main result, Theorem 5.16, shows that the relative error in energy of the diffractive geometric optics approximation tends to zero as $\varepsilon \rightarrow 0$ for amplitudes $a \in H^{1}$. The proof requires stability in energy for $P^{\varepsilon}$ expressed in the proof of Theorem 5.8 and a simple estimate for the Schrödinger equation. Its proof is left to the reader. 
Proposition 5.15. There is a constant $C>0$ so that the approximate solutions (5.36) with $a \in C\left(\left[0, \infty\left[; H^{1}\left(\mathbb{R}^{N}\right)\right)\right.\right.$, a solution of $(5.37)$, satisfy

$$
\sup _{0 \leq t<\infty} \sup _{|\alpha| \leq 1}\left\|\left(\varepsilon \partial_{t, x}\right)^{\alpha} v_{\text {approx }}^{\varepsilon}(t)\right\|_{L^{2}\left(\mathbb{R}^{N}\right)} \leq C \sup _{|\alpha| \leq 1}\left\|\left(\varepsilon \partial_{t, x}\right)^{\alpha} v_{\text {approx }}^{\varepsilon}(0)\right\|_{L^{2}\left(\mathbb{R}^{N}\right)} .
$$

The operators $\varepsilon \partial_{t, x}$ in the main result encode the length scale of the oscillations.

Theorem 5.16. Assume the ray average hypothesis in Definition 5.2 is satisfied. For $a_{0} \in H^{1}\left(\mathbb{R}^{N}\right)$, with $a_{0} \neq 0$, define $u^{\varepsilon}$ to be the solution of $P^{\varepsilon} u^{\varepsilon}=0$ with initial data

$u^{\varepsilon}(0, x)=e^{2 \pi i \theta_{0} . x / \varepsilon} a_{0}(x) \psi_{n}\left(x / \varepsilon, \theta_{0}\right), \quad \partial_{t} u^{\varepsilon}(0, x)=\frac{2 \pi i \omega}{\varepsilon} e^{2 \pi i \theta_{0} \cdot x / \varepsilon} \psi_{n}\left(x / \varepsilon, \theta_{0}\right) a_{0}(x)$.

Then, for any $T>0$, there exists $C>0$ such that, for any $0<\varepsilon \leq 1$, the exact solution satisfies

$$
0<C<\sup _{0 \leq t \leq T / \varepsilon}\left\|\varepsilon \partial_{t, x} u^{\varepsilon}(t)\right\|_{L^{2}\left(\mathbb{R}^{N}\right)} \leq 1 / C
$$

Suppose a satisfies (5.37) with $a(0, \cdot)=a_{0}$, and $v_{\text {approx }}^{\varepsilon}$ is defined by (5.36). Then

$$
\lim _{\varepsilon \rightarrow 0} \sup _{0 \leq t \leq T / \varepsilon} \sup _{|\alpha| \leq 1}\left\|\left(\varepsilon \partial_{t, x}\right)^{\alpha}\left(u^{\varepsilon}(t)-v_{\text {approx }}^{\varepsilon}(t)\right)\right\|_{L^{2}\left(\mathbb{R}^{N}\right)}=0 .
$$

Proof. For any challenge number $\delta>0$, choose $a_{0}^{\delta} \in C_{0}^{\infty}\left(\mathbb{R}^{N}\right)$ so that

$$
\left\|a_{0}-a_{0}^{\delta}\right\|_{H^{1}\left(\mathbb{R}^{N}\right)}<\delta .
$$

For the associated exact and approximate solutions $u_{\delta}^{\varepsilon}$ and $v_{\delta \text {,approx }}^{\varepsilon}$ we have proved that

$$
\sup _{0 \leq t \leq T / \varepsilon} \sup _{|\alpha| \leq 1}\left\|\left(\varepsilon \partial_{t, x}\right)^{\alpha}\left(u_{\delta}^{\varepsilon}(t)-v_{\delta, \text { approx }}^{\varepsilon}(t)\right)\right\|_{L^{2}\left(\mathbb{R}^{N}\right)} \leq C(\delta) \varepsilon^{1-\beta}
$$

Applying (3.35) to $u^{\varepsilon}-u_{\delta}^{\varepsilon}$ shows that

$$
\sup _{0 \leq t \leq T / \varepsilon} \sup _{|\alpha| \leq 1}\left\|\left(\varepsilon \partial_{t, x}\right)^{\alpha}\left(u^{\varepsilon}(t)-u_{\delta}^{\varepsilon}(t)\right)\right\|_{L^{2}\left(\mathbb{R}^{N}\right)} \leq C \delta
$$

Similarly, Proposition 5.15 shows that

$$
\sup _{0 \leq t \leq T / \varepsilon} \sup _{|\alpha| \leq 1}\left\|\left(\varepsilon \partial_{t, x}\right)^{\alpha}\left(v_{\text {approx }}^{\varepsilon}(t)-v_{\delta, \text { approx }}^{\varepsilon}(t)\right)\right\|_{L^{2}\left(\mathbb{R}^{N}\right)} \leq C \delta .
$$

The three last estimates together with the triangle inequality imply that

$$
\sup _{0 \leq t \leq T / \varepsilon} \sup _{|\alpha| \leq 1}\left\|\left(\varepsilon \partial_{t, x}\right)^{\alpha}\left(u^{\varepsilon}(t)-v_{\text {approx }}^{\varepsilon}(t)\right)\right\|_{L^{2}\left(\mathbb{R}^{N}\right)} \leq C(\delta) \varepsilon^{1-\beta}+C_{2} \delta,
$$

with $C_{2}$ independent of $\varepsilon, \delta$. Letting $\varepsilon \rightarrow 0$ yields

$$
\lim _{\varepsilon \rightarrow 0} \sup _{0 \leq t \leq T / \varepsilon}\left\|\varepsilon \partial_{t, x}\left(u^{\varepsilon}(t)-v_{\text {approx }}^{\varepsilon}(t)\right)\right\|_{L^{2}\left(\mathbb{R}^{N}\right)} \leq C_{2} \delta .
$$

Since $\delta>0$ is arbitrary, this completes the proof. 
Example 5.17. Using Theorem 5.16, one finds approximate solutions to the oscillatory initial value problem (5.33) with rough amplitudes

$$
b, c \in H^{1}\left(\mathbb{R}^{N}\right)
$$

and error $o(1)$ in energy as $\varepsilon \rightarrow 0$. It suffices to take as approximate solution

$$
\sum_{ \pm} e^{2 \pi i S^{ \pm} / \varepsilon} \psi_{n}\left(x / \varepsilon, \theta_{0}\right) a^{ \pm}(\varepsilon t, x \mp \mathcal{V} t)
$$

where $a^{ \pm}$are the unique solutions of the Schrödinger equation

$$
\left( \pm 4 \pi i \omega \partial_{\mathcal{T}} \mp \omega \nabla_{\theta}^{2} \omega\left(\partial_{x}, \partial_{x}\right)+\widetilde{\gamma}^{ \pm}(x)\right) a^{ \pm}=0,
$$

with initial values $a^{ \pm}(0, \cdot) \in H^{1}\left(\mathbb{R}^{N}\right)$ determined from,

$$
a^{+}(0, \cdot)+a^{-}(0, \cdot)=b, \quad a^{+}(0, \cdot)-a^{-}(0, \cdot)=\frac{c}{2 \pi i \omega} .
$$

\section{REFERENCES}

[1] J.H. Albert, Genericity of simple eigenvalues for elliptic pde's, Proc. A.M.S.48, 413-418 (1975).

[2] G. Allaire, Dispersive limits in the homogenization of the wave equation, Annales de la Faculté des Sciences de Toulouse, vol. XII 415-431(2003).

[3] H. Altug, J. Vuckovic, Experimental demonstration of the slow group velocity of light in twodimensional coupled photonic crystal microcavity arrays, Appl. Phys. Lett. 86, 111102-1 to 111102-3 (2004).

[4] G. Allaire, M. Palombaro, J. Rauch, Diffractive behavior of the wave equation in periodic media: weak convergence analysis, Annali di Matematica Pura e Applicata 188, 561-590, (2009).

[5] M. Bajcsy, A. Zibrov, M. Lukin, Stationary pulses of light in an atomic medium, Nature 426 (2003).

[6] A. Bamberger, B. Engquist, L. Halpern, P. Joly, Parabolic wave equation approximations in heterogenous media, SIAM J. Appl. Math. 48, no. 1, 99-128, (1988).

[7] K. Barrailh, D. Lannes, A general framework for diffractive optics and its applications to lasers with large spectrum and short pulses, SIAM, Journal on Mathematical Analysis 34, no. 3, 636-674, (2003)

[8] A. Bensoussan, J.-L. Lions, G. Papanicolaou, Asymptotic analysis for periodic structures, NorthHolland, Amsterdam (1978).

[9] F. Bloch, Uber die Quantenmechanik der Electronen in Kristallgittern, Z. Phys. 52, pp. 555-600, (1928).

[10] S. Brahim-Otsmane, G. Francfort, F. Murat, Correctors for the homogenization of the wave and heat equations, J. Math. Pures Appl. (9) 71:197-231, (1992).

[11] L. Brillouin, Propagation of Waves in Periodic Structures, Dover, New York (1953).

[12] C. Conca, J. Planchard, M. Vanninathan, Fluids and periodic structures, RMA 38, J. Wiley \& Masson, Paris (1995).

[13] C. Conca, R. Orive, M. Vanninathan, On Burnett coefficients in strongly periodic media, J. Math. Phys. 47 (2006), no. 3, 032902, 11 pp.

[14] P. Donnat, J.-L. Joly, G. Métivier, J. Rauch, Diffractive nonlinear geometric optics, Séminaire sur les Equations aux Dérivées Partielles, 1995-1996, Exp. No. XVII, 25 pp., Ecole Polytechnique, Palaiseau (1996).

[15] P. Donnat, J. Rauch, Modeling the dispersion of light, Singularities and oscillations (Minneapolis, MN, 1994/1995), 17-35, IMA Vol. Math. Appl., 91, Springer, New York (1997).

[16] P. Donnat, J. Rauch, Dispersive nonlinear geometric optics, J. Math. Phys. 38 , no. 3, 1484-1523, (1997). 
[17] E. Dumas, Diffractive optics with curved phases: beam dispersion and transitions between light and shadow, Asymptotic Analysis 38 , n.1, 47-91,(2004).

[18] G. Francfort, F. Murat, Oscillations and energy densities in the wave equation, Comm. Partial Differential Equations 17, 1785-1865 (1992).

[19] P. Gérard, P. Markowich, N. Mauser, F. Poupaud, Homogenization limits and Wigner transforms, Comm. Pure Appl. Math. 50, no. 4, 323-379 (1997).

[20] H. Gersen, T. Karle, R. Engelen, W. Bogaerts, J. Korterik, N. van Hulst, T. Krauss, and L. Kuipers, Real-space observation of ultraslow light in photonic crystal waveguides, Phys. Rev. Lett. 94(2005) 073903-1-073903-4.

[21] L.V. Hau, S. E. Harris, Z. Dutton, C. Behroozi, Light speed reduction to 17 meters per second in an ultracold atomic gas, Nature 397, 594-598, (1999).

[22] J.-L. Joly, G. Métiver, and J. Rauch, Generic rigorous asymptotic expansions for weakly nonlinear multidimensional oscillatory waves, Duke Math J., 70, 373-404, (1993).

[23] J.-L. Joly, G. Métiver, and J. Rauch, Diffractive nonlinear geometric optics with rectification, Indiana U. Math. J. 47, 1167-1242, (1998).

[24] T. Kato, Perturbation theory for linear operators, Springer-Verlag, Berlin (1966).

[25] P. Kuchment, Floquet theory for partial differential equations, Operator Theory: Advances and Applications, 60, Birkhäuser Verlag, Basel (1993).

[26] P. Kuchment, The mathematics of photonic crystals, Mathematical modeling in optical science, 207-272, Frontiers Appl. Math., 22, SIAM, Philadelphia (2001).

[27] P. D. Lax, Asymptotic solutions of oscillatory initial value problems, Duke Math. J. 24, 627-646, (1957).

[28] M. Leontovich, V. Fock, Solution of the problem of propagation of electromagnetic waves along the earth's surface by the method of parabolic equation, Acad. Sci. USSR. J. Phys. 10, 13-24, (1946).

[29] M. Reed, B. Simon, Methods of modern mathematical physics, Academic Press, New York (1978).

[30] P. St. J. Russell, Photonic crystal fibers, J. Lightwave. Technol., 24 (12), 4729-4749, (2006).

[31] F. Santosa, W. Symes, A dispersive effective medium for wave propagation in periodic composites. SIAM J. Appl. Math. 51, 984-1005, (1991).

[32] J. Sipe and H. Winful, Nonlinear Schrödinger equations in periodic structure, Optics Letters, 13, 132-133, (1988).

[33] C. de Sterke, and J. Sipe, Envelope-function approach for the electrodynamics of nonlinear periodic structures, PhysRevA., 38, 5149-5165, (1988).

[34] F. Tappert, The parabolic approximation method, in Wave Propagation and Underwater Acoustics (Workshop, Mystic, Conn., 1974), 224-287, Lecture Notes in Phys., Vol. 70, Springer, Berlin (1977).

[35] L. Tartar, H-measures, a new approach for studying homogenization, oscillations and concentration effects in partial differential equations, Proc. of the Royal Soc. Edinburgh, 115A, 193-230, (1990).

[36] Y.A. Vlasov, S. Petit, G. Klein, B. Hönerlage, and C. Hirlmann, Femtosecond measurements of the time of flight of photons in a three-dimensional photonic crystal, Phys. Rev. E 60, 1030-1035, (1999).

[37] C. Wilcox, Theory of Bloch waves, J. Analyse Math. 33, 146-167, (1978) 